TRANSACTIONS OF THE

AMERICAN MATHEMATICAL SOCIETY

Volume 360, Number 3, March 2008, Pages 1223-1264

S 0002-9947(07)04176-1

Article electronically published on October 23, 2007

\title{
GENERALIZED SEIFERT SURFACES AND SIGNATURES OF COLORED LINKS
}

\author{
DAVID CIMASONI AND VINCENT FLORENS
}

\begin{abstract}
In this paper, we use 'generalized Seifert surfaces' to extend the Levine-Tristram signature to colored links in $S^{3}$. This yields an integral valued function on the $\mu$-dimensional torus, where $\mu$ is the number of colors of the link. The case $\mu=1$ corresponds to the Levine-Tristram signature. We show that many remarkable properties of the latter invariant extend to this $\mu$-variable generalization: it vanishes for achiral colored links, it is 'piecewise continuous', and the places of the jumps are determined by the Alexander invariants of the colored link. Using a 4-dimensional interpretation and the Atiyah-Singer $G$ signature theorem, we also prove that this signature is invariant by colored concordance, and that it provides a lower bound for the 'slice genus' of the colored link.
\end{abstract}

\section{INTRODUCTION}

Several notions related to knots do not extend naturally and uniquely to links. For example, the fact that two oriented links are isotopic can be understood in different ways: one might require the isotopy to satisfy some condition, e.g. to respect an order on the components of the links. Here is another interesting example. A knot in $S^{3}$ is said to be slice if it bounds a smooth disc in the 4-ball, or equivalently, if it is the cross-section of a smooth 2 -sphere in $S^{4}$. This notion of sliceness for knots admits different generalizations to links. According to Fox [11, a link is slice in the ordinary sense if it is the cross-section of a single smooth 2-sphere in $S^{4}$. It is slice in the strong sense if each of its components is such a cross-section for disjoint 2-spheres in $S^{4}$.

One way to simultaneously take into account this variety of possible generalizations is to consider so-called colored links. Roughly speaking, a $\mu$-colored link is an oriented link in $S^{3}$ whose components are endowed with some integer in $\{1, \ldots, \mu\}$ called the color of the component. Two colored links are isotopic if there is an isotopy between them which respects the color and orientation of each component. We shall say that a $\mu$-colored link is slice if there exists $\mu$ disjoint smooth spheres $S_{1}, \ldots, S_{\mu}$ in $S^{4}$ such that the sublink of color $i$ is a cross-section of $S_{i}$. Of course, a 1-colored link is nothing but an oriented link, and it is slice as a 1-colored link if it is slice in the ordinary sense. At the other end of the spectrum, a $\nu$-component

Received by the editors May 6, 2005 and, in revised form, August 23, 2005.

2000 Mathematics Subject Classification. Primary 57M25.

Key words and phrases. Colored link, Seifert surface, Levine-Tristram signature, slice genus.

The first author was supported by the Swiss National Science Foundation.

The second author was supported by Marie-Curie, MCHF-2001-0615.

(C)2007 American Mathematical Society Reverts to public domain 28 years from publication 
$\nu$-colored link is an ordered link. It is slice as a $\nu$-colored link if it is slice in the strong sense.

Many classical invariants of oriented links, such as the Alexander polynomial and the Levine-Tristram signature, can be constructed using Seifert surfaces. In this paper, we introduce generalized Seifert surfaces for colored links. We use them, inter alia, to extend the Levine-Tristram signature from oriented links to $\mu$-colored links. This yields an integral valued function on the $\mu$-dimensional torus. Among other results (see the paragraph below), we show that this function vanishes almost everywhere if the $\mu$-colored link is slice.

Throughout the paper, all the links are assumed to be smooth and oriented.

The Levine-Tristram signature. Let $V$ be a Seifert matrix for a link $L$ in $S^{3}$. Then, $A(t)=V-t V^{T}$ is a presentation matrix of the Alexander $\mathbb{Z}\left[t^{ \pm 1}\right]$-module of $L$. In particular, the Alexander polynomial $\Delta_{L}$ of $L$ is given by the determinant of $A(t)$. If $\omega \neq 1$ is a unit modulus complex number, then $H(\omega)=(1-\bar{\omega}) A(\omega)$ is a Hermitian matrix whose signature $\sigma_{L}(\omega)$ and nullity $\eta_{L}(\omega)$ do not depend on the choice of $V$. This yields integral valued functions $\sigma_{L}$ and $\eta_{L}$ defined on $S^{1} \backslash\{1\}$. In the case of $\omega=-1$, this signature was first defined by Trotter [33] and studied by Murasugi 24]. The more general formulation is due to Levine [19] and Tristram 32 , and referred to as the Levine-Tristram signature.

The functions $\sigma_{L}$ and $\eta_{L}$ are easily seen to be locally constant on the complement in $S^{1} \backslash\{1\}$ of the roots of $\Delta_{L}$. Also, $\eta_{L}$ is related to the first Betti number of the finite cyclic coverings of the exterior of $L$. Moreover, when restricted to roots of unity of prime order, the signature and nullity are concordance invariants. (The case of $\omega=-1$ is due to Murasugi, and Tristram extended it to any $\omega$ of prime order.) Finally, the so-called Murasugi-Tristram inequality imposes a condition, expressed in terms of the values of $\sigma_{L}$ and $\eta_{L}$, on the Betti numbers of a smooth oriented surface in $B^{4}$ spanning $L$. This inequality implies in particular that if $L$ is slice in the strong sense [11, then $\sigma_{L}$ vanishes at roots of unity of prime order.

At that point, all the methods of demonstration were purely 3-dimensional. A new light was shed on this theory in the early seventies. Building on ideas of Rokhlin 28, Viro 34 was able to interpret the Levine-Tristram signature as a 4 -dimensional object. Indeed, he showed that for all rational values of $\omega, \sigma_{L}(\omega)$ coincides with the signature of an intersection form related to a cyclic cover of $B^{4}$ branched along a Seifert surface for $L$ pushed in the interior of $B^{4}$. This 4dimensional approach was used by Kauffman and Taylor [18 to obtain a short proof of the Murasugi-Tristram inequality, in the case $\omega=-1$. They were also able to show the following inequality: if $P$ is a closed oriented smooth surface in $S^{4}$ that intersects the standardly embedded 3 -sphere in $L$, then

$$
\left|\sigma_{L}(-1)\right| \leq \operatorname{genus}(P)+\min \left(0, \eta_{L}(-1)-\beta_{0}(P)+1\right),
$$

where $\beta_{0}(P)$ denotes the number of connected components of $P$. In particular, if there exists such a surface of genus 0 (that is, according to [11, if $L$ is slice in the ordinary sense), then $\sigma_{L}(-1)=0$. This 4-dimensional interpretation was used with great success by several authors [12, 16, 20, 21, 31. See also [2, 10, 13, 14.

Paper outline and statement of the results. The aim of this paper is to generalize the Levine-Tristram signature to colored links. A $\mu$-colored link $L=$ $L_{1} \cup \cdots \cup L_{\mu}$ is an oriented link in $S^{3}$ together with a surjective map assigning to each component of $L$ a color in $\{1, \ldots, \mu\}$. The sublink $L_{i}$ is constituted by the 
components of $L$ with color $i$ for $i=1, \ldots, \mu$. By isotopy of colored links, we mean orientation and color-preserving isotopy. Note that a 1-colored link is an ordinary link, and setting $\mu=1$ in this article gives back the known results.

In Section 2, we consider generalized Seifert surfaces called $C$-complexes. Roughly speaking, a C-complex for a $\mu$-colored link $L$ consists of a collection of Seifert surfaces $S_{1}, \ldots, S_{\mu}$ for the sublinks $L_{1}, \ldots, L_{\mu}$ that intersect only along clasps. Associated to a C-complex are so-called generalized Seifert matrices. We use them to define a matrix $A\left(t_{1}, \ldots, t_{\mu}\right)$ with coefficients in $\Lambda_{\mu}=\mathbb{Z}\left[t_{1}^{ \pm 1}, \ldots, t_{\mu}^{ \pm 1}\right]$. Of course, this matrix depends on the choice of the C-complex for $L$. However, if $\left(\omega_{1}, \ldots, \omega_{\mu}\right)$ is an element of the $\mu$-dimensional torus $S^{1} \times \cdots \times S^{1} \subset \mathbb{C}^{\mu}$ with $\omega_{i} \neq 1$, then the matrix

$$
H\left(\omega_{1}, \ldots, \omega_{\mu}\right)=\prod_{i=1}^{\mu}\left(1-\bar{\omega}_{i}\right) \cdot A\left(\omega_{1}, \ldots, \omega_{\mu}\right)
$$

is Hermitian and its signature and nullity are independant of the choice of the C-complex for $L$. (We use some 'generalized S-equivalence'; see Lemma 2.2 and Theorem 2.1) This allows one to define the signature and the nullity of the $\mu$ colored link $L$ as functions

$$
\sigma_{L}, \eta_{L}:\left(S^{1} \backslash\{1\}\right)^{\mu} \longrightarrow \mathbb{Z}
$$

Note that this was done by Cooper [6, 7] in the case of a 2-colored link with 2 components. The restriction of these functions to the diagonal specializes to the Tristram-Levine invariants as follows (see Proposition 2.5).

Proposition. Let $L=L_{1} \cup \cdots \cup L_{\mu}$ be a $\mu$-colored link, and let $L^{\prime}$ be the underlying link. For all $\omega$ in $S^{1} \backslash\{1\}$,

$$
\sigma_{L}(\omega, \ldots, \omega)=\sigma_{L^{\prime}}(\omega)+\sum_{i<j} l k\left(L_{i}, L_{j}\right) \quad \text { and } \quad \eta_{L}(\omega, \ldots, \omega)=\eta_{L^{\prime}}(\omega),
$$

where lk denotes the linking number in $S^{3}$.

This result can be quite useful. Indeed, it is often easier to compute a multivariable signature (corresponding to a well-chosen coloring) and to evaluate it on the diagonal, than to directly compute the Levine-Tristram invariant.

Several interesting properties of the Levine-Tristram invariants extend to our functions $\sigma_{L}$ and $\eta_{L}$. For example, they are additive with respect to disjoint and connected sum. Moreover, $\sigma_{L}$ vanishes if the colored link $L$ is isotopic to its mirror image (see Corollary 2.11).

Section 3 is devoted to the study of the natural $\mathbb{Z}^{\mu}$-covering $\widetilde{X} \rightarrow X$ of the exterior of $L$ induced by the coloring of $L$. We show that C-complexes provide a nice geometrical description of this covering, and deduce a presentation of the Alexander $\Lambda_{\mu}$-module $H_{1}(\tilde{X})$ in terms of the generalized Seifert forms (see Theorem 3.2). In particular, if $\Lambda_{\mu}^{\prime}$ denotes the localization of the ring $\Lambda_{\mu}$ with respect to the multiplicative system generated by $t_{i}-1$ for $i=1, \ldots, \mu$, we establish the following result (Corollary 3.6):

Theorem. Let $L$ be a $\mu$-colored link. Consider a $C$-complex $S$ for $L$ such that $S_{i}$ is connected for all $i$ and $S_{i} \cap S_{j}$ is non-empty for all $i \neq j$. Then the corresponding matrix $A\left(t_{1}, \ldots, t_{\mu}\right)$ is a presentation matrix of the $\Lambda_{\mu}^{\prime}$-module $H_{1}(\widetilde{X}) \otimes_{\Lambda_{\mu}} \Lambda_{\mu}^{\prime}$. 
In particular, the Alexander polynomial of $L$ is equal to the determinant of the matrix $A\left(t_{1}, \ldots, t_{\mu}\right)$ up to multiplication by $t_{i}-1$. (This latter result was obtained by the first author in [4 using local relations on a diagram.) This theorem implies the following characterization of the discontinuities of $\sigma_{L}$ and $\eta_{L}$ (Theorem 4.1).

Theorem. Let $E_{r}(L) \subset \Lambda_{\mu}$ be the $r^{\text {th }}$ Alexander ideal of $L$, and set

$$
\Sigma_{r}=\left\{\omega \in S^{1} \times \cdots \times S^{1} \subset \mathbb{C}^{\mu} \mid p(\omega)=0 \text { for all } p \in E_{r-1}(L)\right\} .
$$

This yields a finite sequence of algebraic subvarieties of the torus $S^{1} \times \cdots \times S^{1}$. Setting $\Sigma_{r}^{*}=\Sigma_{r} \cap\left(S^{1} \backslash\{1\}\right)^{\mu}$, we obtain a finite sequence $\left(S^{1} \backslash\{1\}\right)^{\mu}=\Sigma_{0}^{*} \supset \Sigma_{1}^{*} \supset$ $\cdots \supset \Sigma_{\ell-1}^{*} \supset \Sigma_{\ell}^{*}=\emptyset$ such that, for all $r, \eta_{L}$ is equal to $r$ on $\Sigma_{r}^{*} \backslash \Sigma_{r+1}^{*}$, and $\sigma_{L}$ is locally constant on $\Sigma_{r}^{*} \backslash \Sigma_{r+1}^{*}$.

This 'piecewise continuity' behavior was first observed by Levine 21] for closely related invariants. The most interesting point of our result is the relation to the Alexander invariants. Note that even if the Alexander polynomial is zero, the signature is locally constant.

In Section [5, we build on an idea of Conway [5] to show that the signature satisfies several 'local relations.' (We refer to Theorem [5.1 for a precise statement.) In many cases, this leads to a purely combinatorial computation of the signature from a diagram of the corresponding colored link.

The following section deals with a 4-dimensional interpretation of $\sigma_{L}(\omega)$ and $\eta_{L}(\omega)$ for all $\omega=\left(\omega_{1}, \ldots, \omega_{\mu}\right)$ with rational coordinates in $\left(S^{1} \backslash\{1\}\right)^{\mu}$. We consider a union $F$ of connected surfaces $F_{1}, \ldots, F_{\mu}$ smoothly embedded in $B^{4}$ such that $\partial F_{i} \subset \partial B^{4}=S^{3}$ is the sublink $L_{i}$, and the pairwise intersections of the $F_{i}$ 's are transverse (along a finite number of points). The first homology of the exterior $W_{F}$ in $B^{4}$ of such a 'spanning surface' is free of rank $\mu$. Therefore, any rational point $\omega \in\left(S^{1} \backslash\{1\}\right)^{\mu}$ determines a character of $H_{1}\left(W_{F}\right)$ of finite order. This character induces twisted homology $\mathbb{C}$-vector spaces, denoted by $H_{*}^{\omega}\left(W_{F} ; \mathbb{C}\right)$, and a Hermitian twisted intersection form $\varphi_{F}^{\omega}: H_{2}^{\omega}\left(W_{F} ; \mathbb{C}\right) \times H_{2}^{\omega}\left(W_{F} ; \mathbb{C}\right) \rightarrow \mathbb{C}$. We obtain the following result (see Lemma 6.3 Lemma 6.4 and Proposition 6.5).

Proposition. Consider a connected $C$-complex $S \subset S^{3}$ for a $\mu$-colored link L. Let $F \subset B^{4}$ be the spanning surface for $L$ obtained by pushing $S$ in $B^{4}$. For any rational point $\omega \in\left(S^{1} \backslash\{1\}\right)^{\mu}, H(\omega)$ is a matrix for $\varphi_{F}^{\omega}$.

The proof follows from an explicit geometrical description of the finite abelian coverings of $W_{F}$. We also make use of the work of Sakuma [29] for the study of cyclic quotients of these coverings. The Atiyah-Singer $G$-signature theorem [1] implies that the signature of $\varphi_{F}^{\omega}$ does not depend on the choice of the spanning surface $F$ for $L$. Moreover, the nullity of $\varphi_{F}^{\omega}$ is closely related to the twisted homology of the exterior $X$ of $L$ in $S^{3}$. This leads to the following result (Theorem 6.1).

Theorem. Let $L$ be a $\mu$-colored link with exterior $X$, and $F$ be a spanning surface for $L$ in $B^{4}$. For all rational points $\omega$ in $\left(S^{1} \backslash\{1\}\right)^{\mu}$,

$$
\begin{aligned}
& \sigma_{L}(\omega)=\operatorname{sign}\left(\varphi_{F}^{\omega}\right), \\
& \eta_{L}(\omega)=\operatorname{dim} H_{1}^{\omega}(X ; \mathbb{C})=\operatorname{null}\left(\varphi_{F}^{\omega}\right)+\operatorname{dim} H_{1}^{\omega}\left(W_{F} ; \mathbb{C}\right)-\operatorname{dim} H_{3}^{\omega}\left(W_{F} ; \mathbb{C}\right) .
\end{aligned}
$$

As a consequence, if the colored link $L=L_{1} \cup \cdots \cup L_{\mu}$ satisfies $l k\left(L_{i}, L_{j}\right)=0$ for all $i \neq j$, then $\sigma_{L}(\omega)$ and $\eta_{L}(\omega)$ coincide with the invariants considered by the 
second author in [9]. It also relates our signature function to various invariants introduced by Gilmer [12, Smolinsky [31] and Levine [21]. Note that the first equality concerning the nullity is closely related to Libgober's [22].

Combining our construction with [12, we obtain the following formula for the Casson-Gordon invariant of a 3-manifold (Theorem 6.7).

Theorem. Let $M$ be the 3-manifold obtained by surgery on a framed link $L$ with $\nu$ components and linking matrix $\Lambda$. Let $\chi: H_{1}(M) \rightarrow \mathbb{C}^{*}$ be the character mapping the meridian of the $i^{\text {th }}$ component of $L$ to $\alpha^{n_{i}}$, where $\alpha=e^{2 i \pi / q}$ and $n_{i}$ is an integer coprime to $q$. Consider $L$ as a $\nu$-colored link and set $\omega=\left(\alpha^{n_{1}}, \ldots, \alpha^{n_{\nu}}\right)$. Then, the Casson-Gordon invariant of the pair $(M, \chi)$ is given by

$$
\sigma(M, \chi)=\left(\sigma_{L}(\omega)-\sum_{i<j} \Lambda_{i j}\right)-\operatorname{sign}(\Lambda)+\frac{2}{q^{2}} \sum_{i, j}\left(q-n_{i}\right) n_{j} \Lambda_{i j} .
$$

The 4-dimensional point of view developed in Section 6 makes it possible to prove several results that would have been horrendous to check using only 3-dimensional techniques. Let us denote by $T_{\mathcal{P}}^{\mu}$ the dense subset of $S^{1} \times \cdots \times S^{1}$ given by the elements of the form $\omega=\left(\omega_{1}, \ldots, \omega_{\mu}\right)$ which satisfy the following condition: there exists a prime $p$ such that for all $i$, the order of $\omega_{i}$ is a power of $p$.

Theorem. For all $\omega \in T_{\mathcal{P}}^{\mu}, \sigma_{L}(\omega)$ and $\eta_{L}(\omega)$ are invariant by colored concordance.

We also extend the Murasugi-Tristram inequality to the case of surfaces that intersect transversally (Theorem 7.2 ). This can be viewed as a specialization of [12, Theorem 4.1]. Finally, we show the following generalization of the Kauffman-Taylor inequality $(\star)$.

Theorem. Consider a colored link $L=L_{1} \cup \cdots \cup L_{\mu}$. Let us assume that there exists a smooth oriented closed surface $P=P_{1} \sqcup \cdots \sqcup P_{\mu}$ in $S^{4}$ such that $P_{i} \cap S^{3}=L_{i}$ for all $i$, where $S^{3}$ denotes the standard embedding of the 3 -sphere in $S^{4}$. Then, for all $\omega$ in $T_{\mathcal{P}}^{\mu}$,

$$
\left|\sigma_{L}(\omega)\right| \leq \operatorname{genus}(P)+\min \left(0, \eta_{L}(\omega)-\mu+1\right) .
$$

If there exists such a surface $P$ of genus zero, we say that $L$ is a slice colored link. As an immediate corollary, we get: if $L$ is a slice $\mu$-colored link, then $\sigma_{L}(\omega)=0$ and $\eta_{L}(\omega) \geq \mu-1$ for all $\omega$ in $T_{\mathcal{P}}^{\mu}$. This notion of 'sliceness' is in fact a natural generalization of the definitions of Fox [11] stated above. Indeed, a 1-colored link is slice if and only if it is slice in the ordinary sense. On the other hand, a $\nu$ component link is slice as a $\nu$-colored link if and only if it is slice in the strong sense. What we get is a spectrum of sliceness notions ranging from the ordinary sense to the strong sense. To each coloring of a given link, there corresponds one notion of sliceness, and one signature function. This function vanishes if the link is slice in the corresponding sense.

It should be pointed out that all the results of Sections 2 to 4 hold for colored links in an arbitrary $\mathbb{Z}$-homology 3 -sphere. Sections 6 and 7 also extend to this setting, provided the homology sphere bounds a contractible 4-manifold.

Finally, let us mention that the results of the present paper have been successfully applied to the study of the topology of real algebraic plane curves. Indeed, S. Yu. Orevkov implemented an algorithm computing the generalized Seifert matrices (and therefore, the signature and nullity functions) of a colored link given as the closure of a colored braid. Using this computer program, the generalized 
Murasugi-Tristram inequality (Theorem 7.2 below), and his method developed in 25], he was able to complete the classification up to isotopy of M-curves of degree 9 with 4 nests. We refer to the upcoming paper [27] for details.

\section{Definition AND Basic PRoperties of $\sigma_{L}$ AND $\eta_{L}$}

The aim of this section is to define the signature and nullity of a colored link as a natural generalization of the Levine-Tristram signature of an oriented link.

2.1. C-complexes. Recall that a Seifert surface for a link in $S^{3}$ is a connected compact oriented surface smoothly embedded in $S^{3}$ that has the link as its oriented boundary. The notion of C-complex, as introduced in [7] and 4, is a generalization of Seifert surfaces to colored links.

Definition. A $C$-complex for a $\mu$-colored link $L=L_{1} \cup \cdots \cup L_{\mu}$ is a union $S=$ $S_{1} \cup \cdots \cup S_{\mu}$ of surfaces in $S^{3}$ such that:

(i) for all $i, S_{i}$ is a Seifert surface for $L_{i}$ (possibly disconnected, but with no closed components);

(ii) for all $i \neq j, S_{i} \cap S_{j}$ is either empty or a union of clasps (see Figure 1);

(iii) for all $i, j, k$ pairwise distinct, $S_{i} \cap S_{j} \cap S_{k}$ is empty.

The existence of a C-complex for a colored link is fairly easy to establish; see 4. Lemma 1]. In the case $\mu=1$, a C-complex for $L$ is nothing but a (possibly disconnected) Seifert surface for the link $L$. Let us now define the corresponding generalization of the Seifert form. Let $N_{i}=S_{i} \times[-1,1]$ be a bicollar neighborhood of $S_{i}$. Given a sign $\varepsilon_{i}= \pm 1$, let $S_{i}^{\varepsilon_{i}}$ be the translated surface $S_{i} \times\left\{\varepsilon_{i}\right\} \subset N_{i}$. Also, let $X$ be the complement of an open tubular neighborhood of $L$, and let $Y$ be the complement of $\bigcup_{i=1}^{\mu}$ int $N_{i}$ in $X$. Given a sequence $\varepsilon=\left(\varepsilon_{1}, \ldots, \varepsilon_{\mu}\right)$ of \pm 1 's, set

$$
S^{\varepsilon}=\bigcup_{i=1}^{\mu} S_{i}^{\varepsilon_{i}} \cap Y .
$$

Since all the intersections are clasps, there is an obvious homotopy equivalence between $S$ and $S^{\varepsilon}$ inducing an isomorphism $H_{1}(S) \rightarrow H_{1}\left(S^{\varepsilon}\right)$. Let $i^{\varepsilon}: H_{1}(S) \rightarrow$ $H_{1}\left(S^{3} \backslash S\right)$ be the composition of this isomorphism with the inclusion homomorphism $H_{1}\left(S^{\varepsilon}\right) \rightarrow H_{1}\left(S^{3} \backslash S\right)$. Finally, let

$$
\alpha^{\varepsilon}: H_{1}(S) \times H_{1}(S) \rightarrow \mathbb{Z}
$$

be the bilinear form given by $\alpha^{\varepsilon}(x, y)=l k\left(i^{\varepsilon}(x), y\right)$, where $l k$ denotes the linking number. Fix a basis of $H_{1}(S)$ and denote by $A_{S}^{\varepsilon}$ (or simply by $A^{\varepsilon}$ ) the matrix of $\alpha^{\varepsilon}$. Of course, if $\mu=1$, then $\alpha^{-}$is the usual Seifert form and $A^{-}$the usual Seifert matrix. Note that for all $\varepsilon, A^{-\varepsilon}$ is equal to $\left(A^{\varepsilon}\right)^{T}$, the transpose of the matrix $A^{\varepsilon}$.

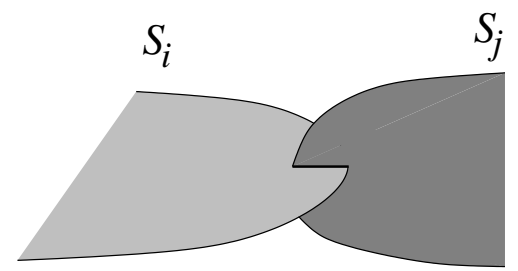

Figure 1. A clasp intersection. 


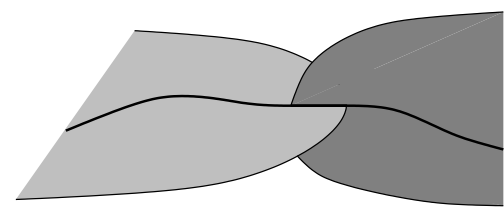

Figure 2. A loop crossing a clasp.

For computational purposes, the following alternative definition of $i^{\varepsilon}$ is more convenient. A 1-cycle in a C-complex is called a loop if it is an oriented simple closed curve which behaves as illustrated in Figure 2 whenever it crosses a clasp. Clearly, there exists a collection of loops whose homology classes form a basis of $H_{1}(S)$. Therefore, it is possible to define $i^{\varepsilon}$ as follows: for any loop $x, i^{\varepsilon}([x])$ is the class of the 1-cycle obtained by pushing $x$ in the $\varepsilon_{i}$-normal direction off $S_{i}$ for $i=1, \ldots, \mu$. The fact that $x$ is a loop ensures that this can be done continuously along the clasp intersections. We easily check that this definition of $i^{\varepsilon}$ coincides with the intrinsic definition given above.

2.2. The signature and nullity of a colored link. Let $L$ be a $\mu$-colored link. Consider a C-complex $S$ for $L$ and the associated Seifert matrices $A^{\varepsilon}$ with respect to some fixed basis of $H_{1}(S)$. Let $A\left(t_{1}, \ldots, t_{\mu}\right)$ be the matrix with coefficients in $\mathbb{Z}\left[t_{1}, \ldots, t_{\mu}\right]$ defined by

$$
A\left(t_{1}, \ldots, t_{\mu}\right)=\sum_{\varepsilon} \varepsilon_{1} \cdots \varepsilon_{\mu} t_{1}^{\frac{1-\varepsilon_{1}}{2}} \cdots t_{\mu}^{\frac{1-\varepsilon_{\mu}}{2}} A^{\varepsilon},
$$

where the sum is on the $2^{\mu}$ possible sequences $\varepsilon=\left(\varepsilon_{1}, \ldots, \varepsilon_{\mu}\right)$ of \pm 1 's. For $\omega=$ $\left(\omega_{1}, \ldots, \omega_{\mu}\right)$ in $T^{\mu}=S^{1} \times \cdots \times S^{1} \subset \mathbb{C}^{\mu}$, set

$$
H(\omega)=\prod_{i=1}^{\mu}\left(1-\bar{\omega}_{i}\right) A\left(\omega_{1}, \ldots, \omega_{\mu}\right)=\sum_{\varepsilon} \prod_{i=1}^{\mu}\left(1-\bar{\omega}_{i}^{\varepsilon_{i}}\right) A^{\varepsilon} .
$$

Using the fact that $A^{-\varepsilon}=\left(A^{\varepsilon}\right)^{T}$, one easily checks that $H(\omega)$ is a Hermitian matrix. Recall that the eigenvalues of such a matrix $H$ are real. Its signature $\operatorname{sign}(H)$ is defined as the number of positive eigenvalues minus the number of negative eigenvalues. The nullity null $(H)$ is the number of zero eigenvalues of $H$.

Definition. Let $T_{*}^{\mu}$ be the open subset $\left(S^{1} \backslash\{1\}\right)^{\mu}$ of the $\mu$-dimensional torus $T^{\mu} \subset \mathbb{C}^{\mu}$. The signature and nullity of the $\mu$-colored link $L$ are the functions

$$
\sigma_{L}, \eta_{L}: T_{*}^{\mu} \longrightarrow \mathbb{Z}
$$

given by $\sigma_{L}(\omega)=\operatorname{sign}(H(\omega))$ and $\eta_{L}(\omega)=\operatorname{null}(H(\omega))+\beta_{0}(S)-1$, where $\beta_{0}(S)$ denotes the number of connected components of $S$.

By Sylvester's theorem, $\sigma_{L}(\omega)$ and $\eta_{L}(\omega)$ do not depend on the choice of a basis of $H_{1}(S)$.

Theorem 2.1. The signature $\sigma_{L}$ and nullity $\eta_{L}$ do not depend on the choice of the $C$-complex for the colored link L. Hence, they are well-defined as isotopy invariants of the colored link $L$. 

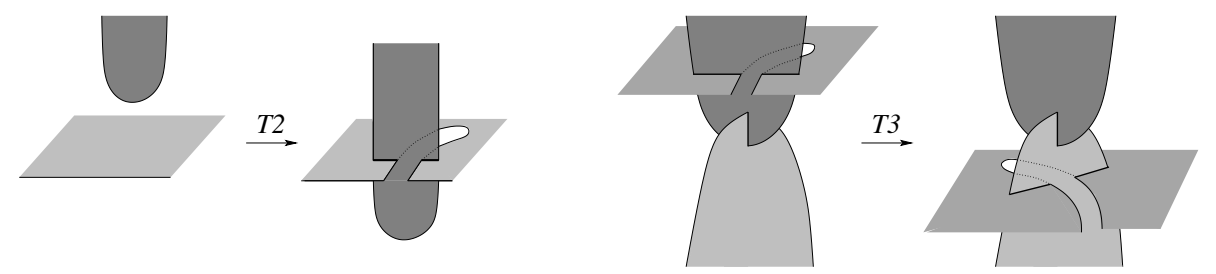

FiguRE 3. The transformations (T2) and (T3) in Lemma 2.2 .

This theorem relies on the following lemma (see [4] for the proof).

Lemma 2.2. Let $S$ and $S^{\prime}$ be $C$-complexes for isotopic colored links. Then, $S$ and $S^{\prime}$ can be transformed into each other by a finite number of the following operations and their inverses:

(T0) ambient isotopy;

(T1) handle attachment on one surface;

(T2) addition of a ribbon intersection, followed by a 'push along an arc' through this intersection (see Figure 3);

(T3) the transformation described in Figure 3 .

Proof of Theorem 2.1. Let $H$ and $H^{\prime}$ be two Hermitian matrices. We shall call $H^{\prime}$ an elementary enlargement of $H$ if

$$
H^{\prime}=\left(\begin{array}{ccc}
H & \xi & 0 \\
\bar{\xi}^{T} & \lambda & \alpha \\
0 & \bar{\alpha} & 0
\end{array}\right)
$$

where $\xi$ is any complex vector, $\lambda$ any real number and $\alpha \in \mathbb{C}^{*} . H$ is called an elementary reduction of $H^{\prime}$. One easily checks that the signature and nullity of a Hermitian matrix are unchanged by elementary enlargements and reductions. By Lemma 2.2, it remains to prove that the transformations (T1) to (T3) of a Ccomplex induce finite sequences of elementary reductions and enlargements on the corresponding Hermitian matrices (or other elementary transformations leaving the signature and nullity unchanged).

(T1) Let $S^{\prime}$ be a C-complex obtained from a C-complex $S$ by a handle attachment on $S_{k}$. If this handle connects two distinct connected components of $S$, then $H_{1}\left(S^{\prime}\right)=H_{1}(S) \oplus \mathbb{Z} y$, where $y$ is a 1 -cycle such that $l k\left(i^{\varepsilon}(y), z\right)=0$ for all $z$ in $H_{1}\left(S^{\prime}\right)$. Hence, the Hermitian matrices $H$ and $H^{\prime}$ corresponding to $S$ and $S^{\prime}$ are related by $H^{\prime}=H \oplus(0)$, so $\operatorname{sign}\left(H^{\prime}\right)=\operatorname{sign}(H)$ and null $\left(H^{\prime}\right)=\operatorname{null}(H)+1$. Since $\beta_{0}\left(S^{\prime}\right)=\beta_{0}(S)-1$, the signature and nullity of $L$ are unchanged. Let us now assume that this handle attachment is performed on one connected component of $S$. In this case, $H_{1}\left(S^{\prime}\right)=H_{1}(S) \oplus \mathbb{Z} x \oplus \mathbb{Z} y$. Moreover, the cycles $x$ and $y$ can be chosen so that the corresponding Seifert matrices satisfy

$$
A_{S^{\prime}}^{\varepsilon}=\left(\begin{array}{ccc}
A_{S}^{\varepsilon} & * & 0 \\
* & * & \pi(-\varepsilon) \\
0 & \pi(\varepsilon) & 0
\end{array}\right), \quad \text { with } \quad \pi(\varepsilon)= \begin{cases}1 & \text { if } \varepsilon_{k}=+1 \\
0 & \text { else. }\end{cases}
$$

Such a choice of $x$ and $y$ is illustrated in Figure 4, The Hermitian matrices $H$ and 


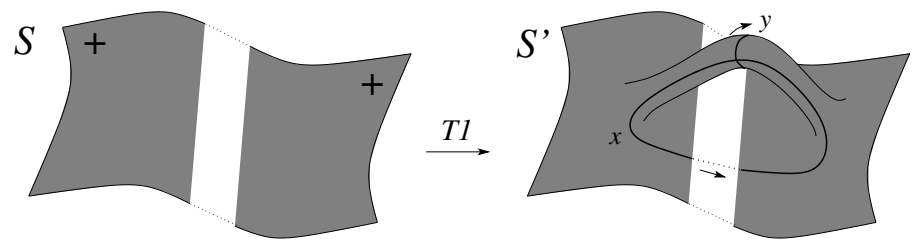

FIGURE 4.

$H^{\prime}$ are related by

$$
H^{\prime}(\omega)=\left(\begin{array}{ccc}
H(\omega) & * & 0 \\
* & * & \alpha \\
0 & \bar{\alpha} & 0
\end{array}\right),
$$

with $\alpha=\left(1-\bar{\omega}_{k}\right) \prod_{i \neq k}\left|1-\omega_{i}\right|^{2}$. Since $H^{\prime}(\omega)$ is Hermitian and $\omega_{i} \neq 1$ for all $i$, $H^{\prime}(\omega)$ is an elementary enlargement of $H(\omega)$.

(T2) Let $S^{\prime}$ be a C-complex obtained from $S$ by the transformation (T2). If this transformation connects two distinct connected components of $S$, then $H_{1}\left(S^{\prime}\right)=$ $H_{1}(S) \oplus \mathbb{Z} z$. As above, we get $H^{\prime}=H \oplus(0)$ and $\beta_{0}\left(S^{\prime}\right)=\beta_{0}(S)-1$, so the signature and nullity are unchanged. On the other hand, if this transformation takes place on one connected component of $S$, then $H_{1}\left(S^{\prime}\right)=H_{1}(S) \oplus \mathbb{Z} w \oplus \mathbb{Z} z$ with $w$ and $z$ as illustrated in Figure 5 .

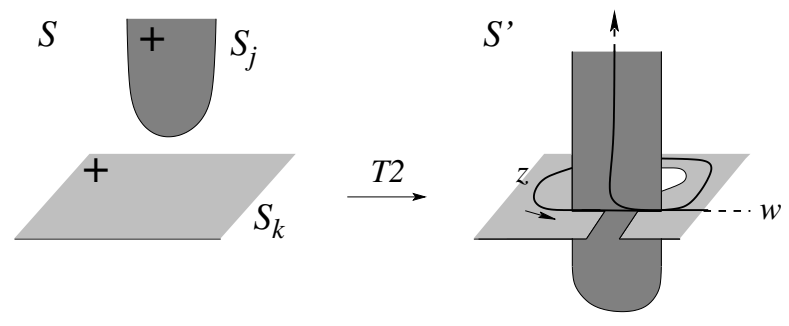

FiguRE 5 .

Therefore,

$$
A_{S^{\prime}}^{\varepsilon}=\left(\begin{array}{ccc}
A_{S}^{\varepsilon} & * & 0 \\
* & * & \delta(-\varepsilon) \\
0 & \delta(\varepsilon) & 0
\end{array}\right), \quad \text { with } \quad \delta(\varepsilon)= \begin{cases}1 & \text { if } \varepsilon_{j}=\varepsilon_{k}=+1 \\
0 & \text { else. }\end{cases}
$$

It follows that the corresponding Hermitian matrix $H^{\prime}(\omega)$ is an elementary enlargement of $H(\omega)$ with $\alpha=\left(1-\bar{\omega}_{j}\right)\left(1-\bar{\omega}_{k}\right) \prod_{i \neq j, k}\left|1-\omega_{i}\right|^{2}$.

(T3) Finally, let $S$ and $S^{\prime}$ be C-complexes related by the move (T3). A similar computation shows that the corresponding Hermitian matrices $H(\omega)$ and $H^{\prime}(\omega)$ are both elementary enlargements of some Hermitian matrix. This concludes the proof.

Example 2.3. If $\mu=1$, then the colored link $L$ is just a link. Furthermore, a C-complex $S$ for $L$ is nothing but a (possibly disconnected) Seifert surface for $L$. Finally, $A^{-}$is a usual Seifert matrix $A$, and $A^{+}=A^{T}$. Hence, the corresponding Hermitian matrix is given by

$$
H(\omega)=(1-\bar{\omega})\left(A^{T}-\omega A\right)=(1-\omega) A+(1-\bar{\omega}) A^{T} .
$$


So if $\mu=1$, the signature of $L$ is the Levine-Tristram signature of the link $L$.
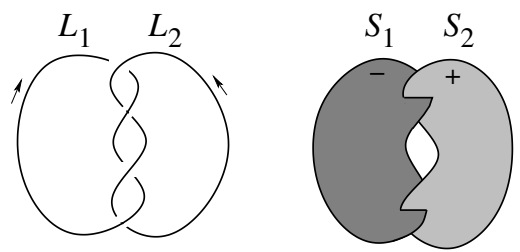

FiguRE 6.

Example 2.4. Consider the 2-colored link $L$ illustrated in Figure 6 A C-complex $S$ for $L$ is also given. We compute $A^{\varepsilon}=(-1)$ if $\varepsilon_{1}=\varepsilon_{2}$, and $A^{\varepsilon}=(0)$ else. Hence,

$$
H\left(\omega_{1}, \omega_{2}\right)=\left(1-\bar{\omega}_{1}\right)\left(1-\bar{\omega}_{2}\right)\left(-1-\omega_{1} \omega_{2}\right)=-2 \Re\left(\left(1-\omega_{1}\right)\left(1-\omega_{2}\right)\right) .
$$

So $\sigma_{L}\left(\omega_{1}, \omega_{2}\right)$ is given by the sign of $-\Re\left(\left(1-\omega_{1}\right)\left(1-\omega_{2}\right)\right)$. Furthermore, $\eta_{L}\left(\omega_{1}, \omega_{2}\right)=$ 1 if $\omega_{1} \omega_{2}=-1$, and $\eta_{L}\left(\omega_{1}, \omega_{2}\right)=0$ else. Let us draw the domain $T_{*}^{2}$ as a square. The value of the function $\sigma_{L}$ can be represented as illustrated in Figure 7. Note that $\sigma_{L}$ and $\eta_{L}$ are constant on the connected components of the complement of the zeroes of $\Delta_{L}\left(t_{1}, t_{2}\right)=t_{1} t_{2}+1$, the Alexander polynomial of $L$. We shall explain this fact in Section 4 .

Proposition 2.5. Let $L=L_{1} \cup \cdots \cup L_{\mu+1}$ be a $(\mu+1)$-colored link. Consider the $\mu$-colored link $L^{\prime}=L_{1}^{\prime} \cup \cdots \cup L_{\mu}^{\prime}$ given by $L_{i}^{\prime}=L_{i}$ for $i<\mu$ and $L_{\mu}^{\prime}=L_{\mu} \cup L_{\mu+1}$. Then, for all $\left(\omega_{1}, \ldots, \omega_{\mu}\right)$ in $T_{*}^{\mu}$,

$$
\begin{aligned}
& \sigma_{L^{\prime}}\left(\omega_{1}, \ldots, \omega_{\mu}\right)=\sigma_{L}\left(\omega_{1}, \ldots, \omega_{\mu}, \omega_{\mu}\right)-l k\left(L_{\mu}, L_{\mu+1}\right), \\
& \eta_{L^{\prime}}\left(\omega_{1}, \ldots, \omega_{\mu}\right)=\eta_{L}\left(\omega_{1}, \ldots, \omega_{\mu}, \omega_{\mu}\right) .
\end{aligned}
$$

Before giving the proof of this proposition, let us point out an interesting consequence: it is possible to compute the signature and nullity of a $\mu$-colored link by considering any finer coloring of the same underlying link. In particular, all the signatures (corresponding to all the possible colorings) can be computed from the signature corresponding to a coloring with the maximal number of colors. This greatly simplifies the computations in many cases, as illustrated by the following (didactic) example.

Example 2.6. Let us try to compute the Levine-Tristram signature of the link $L$ illustrated in Figure 8, One possibility is to choose a Seifert surface for $L$ and to compute the corresponding Seifert matrix.

On the other hand, consider a 3 -colored link $L^{\prime}$ obtained by coloring the components of $L$ with three different colors. There is an obvious contractible C-complex

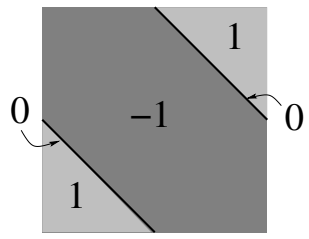

FIGURE 7. 


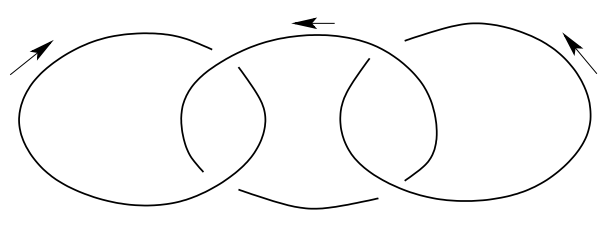

FiguRE 8.

for $L^{\prime}$, so $\sigma_{L^{\prime}}$ is identically zero. By Proposition 2.5, the Levine-Tristram signature of $L$ is given by $\sigma_{L}(\omega)=\sigma_{L^{\prime}}(\omega, \omega, \omega)-2=-2$.

Proof of Proposition 2.5. First, note that it is sufficient to prove this statement when $L_{\mu+1}$ is a knot. Consider a C-complex $S=S_{1} \cup \cdots \cup S_{\mu+1}$ for $L$, and let $\ell$ be the number of clasps in $S_{\mu} \cap S_{\mu+1}$. A certain number of these clasps (say, $\ell_{+}$) induce a positive linking number between $L_{\mu}$ and $L_{\mu+1}$, while the $\ell_{-}$ remaining ones induce a negative linking number. By definition, $\ell=\ell_{+}+\ell_{-}$and $l k\left(L_{\mu}, L_{\mu+1}\right)=\ell_{+}-\ell_{-}$. Using transformation (T2) of Figure 3, it may be assumed that $\ell_{+}$and $\ell_{-}$are positive. Via handle attachment, it may also be assumed that $S_{\mu}$ is connected. Finally, one easily checks that the C-complex $S$ can be chosen so that the knot $L_{\mu+1}$ crosses the $\ell_{-}$negative clasps first, and then the $\ell_{+}$positive ones. This situation is illustrated in Figure 9.

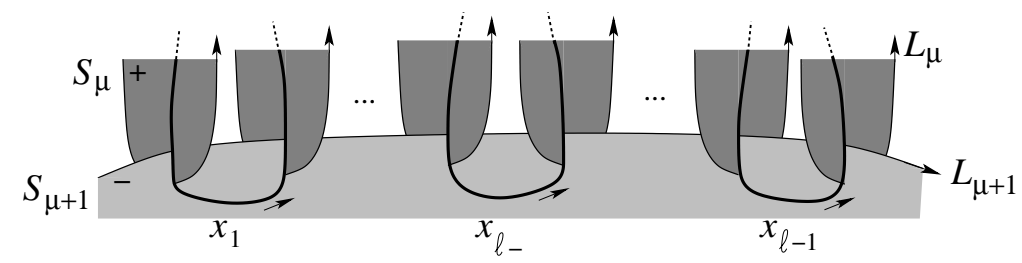

FiguRE 9.

Let $\widetilde{S}$ be the C-complex obtained from $S$ by removing these $\ell$ clasps. Since $S_{\mu}$ and $S_{\mu+1}$ are connected, $H_{1}(S)=\bigoplus_{i=1}^{\ell-1} \mathbb{Z} x_{i} \oplus H_{1}(\widetilde{S})$, with $x_{i}$ the 1-cycles in $S_{\mu} \cup S_{\mu+1}$ depicted above. Fix a basis $\mathcal{B}$ of $H_{1}(\widetilde{S})$. For any sequence $\varepsilon=\left(\varepsilon_{1}, \ldots, \varepsilon_{\mu+1}\right)$ of \pm 1 's, the Seifert matrix $A_{S}^{\varepsilon}$ corresponding to the basis $\left\langle x_{1}, \ldots, x_{\ell-1}\right\rangle \cup \mathcal{B}$ of $H_{1}(S)$ can be written

$$
A_{S}^{\varepsilon}=\left(\begin{array}{cc}
D^{\varepsilon_{\mu} \varepsilon_{\mu+1}} & C^{\varepsilon_{\mu}} \\
\left(C^{-\varepsilon_{\mu}}\right)^{T} & A_{\widetilde{S}}^{\varepsilon}
\end{array}\right) .
$$

Fix an element $\omega^{\prime}=\left(\omega_{1}, \ldots, \omega_{\mu}\right)$ of $T_{*}^{\mu}$. Since $D^{\varepsilon_{\mu} \varepsilon_{\mu+1}}$ only depends on $\varepsilon_{\mu}$ and $\varepsilon_{\mu+1}$, the upper left block of the corresponding Hermitian matrix $H_{S}\left(\omega^{\prime}, \omega_{\mu}\right)$ is given by

$$
\prod_{i=1}^{\mu}\left|1-\omega_{i}\right|^{2}\left(\lambda D+\bar{\lambda} D^{T}+N\right)
$$


where $\lambda=1-\omega_{\mu}, D=D^{--}$and

$(\star) \quad N=\left(D^{-+}-D\right)+\left(D^{-+}-D\right)^{T}=$

$$
\left(\begin{array}{rrrrrrr}
-2 & 1 & & & & & \\
1 & \ddots & \ddots & & & & \\
& \ddots & -2 & 1 & & & \\
& & 1 & 0 & -1 & & \\
& & & -1 & 2 & \ddots & \\
& & & & \ddots & \ddots & -1 \\
& & & & & -1 & 2
\end{array}\right) .
$$

Similarly, the upper right block of $H_{S}\left(\omega^{\prime}, \omega_{\mu}\right)$ is equal to $\prod_{i=1}^{\mu}\left|1-\omega_{i}\right|^{2}\left(\lambda C^{-}+\right.$ $\left.\bar{\lambda} C^{+}\right)$. Finally, observe that each coefficient of $A_{\widetilde{S}}^{\varepsilon}$ is either independant of $\varepsilon_{\mu}$, or independant of $\varepsilon_{\mu+1}$. Therefore, $H_{\widetilde{S}}\left(\omega^{\prime}, \omega_{\mu}\right)=|\lambda|^{2} H_{\widetilde{S}^{\prime}}\left(\omega^{\prime}\right)$, where $\widetilde{S}^{\prime}=S_{1} \cup \cdots \cup$ $S_{\mu-1} \cup \widetilde{S}_{\mu}^{\prime}$ and $\widetilde{S}_{\mu}^{\prime}=\widetilde{S}_{\mu} \sqcup \widetilde{S}_{\mu+1}$. To sum up, we have the equality

$$
H_{S}\left(\omega^{\prime}, \omega_{\mu}\right)=\prod_{i=1}^{\mu}\left|1-\omega_{i}\right|^{2}\left(\begin{array}{cc}
\lambda D+\bar{\lambda} D^{T}+N & \lambda C^{-}+\bar{\lambda} C^{+} \\
\left(\bar{\lambda} C^{-}+\lambda C^{+}\right)^{T} & \prod_{i=1}^{\mu-1}\left|1-\omega_{i}\right|^{-2} H_{\widetilde{S}^{\prime}}\left(\omega^{\prime}\right)
\end{array}\right)
$$

Let us now turn to the $\mu$-colored link $L^{\prime}$. The C-complex $S$ can be transformed into a C-complex $S^{\prime}=S_{1}^{\prime} \cup \cdots \cup S_{\mu}^{\prime}$ for $L^{\prime}$ as follows: set $S_{i}^{\prime}=S_{i}$ for $i<\mu$, and let $S_{\mu}^{\prime}$ be the surface obtained from $S_{\mu} \cup S_{\mu+1}$ by 'smoothing' the $\ell$ clasps as illustrated in Figure 10.

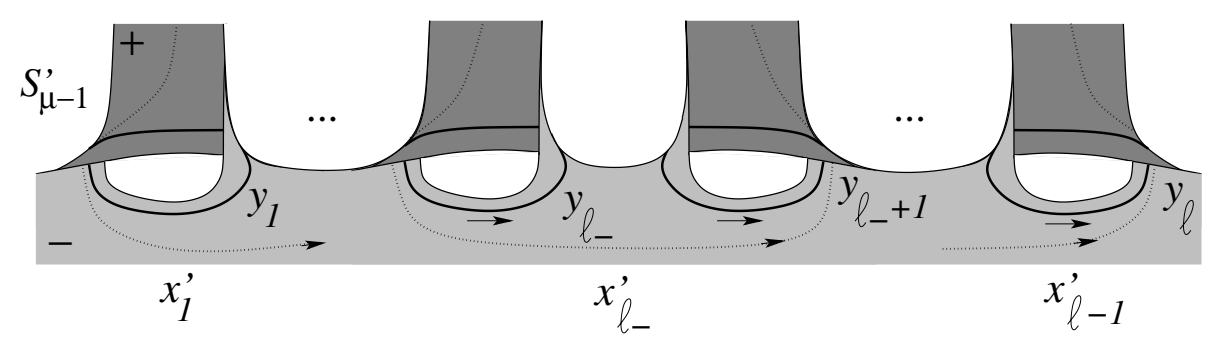

Figure 10.

Basically, each clasp is replaced by two half-twisted bands joining $S_{\mu}$ and $S_{\mu+1}$. This time, $H_{1}\left(S^{\prime}\right)=\bigoplus_{i=1}^{\ell} \mathbb{Z} y_{i} \oplus \bigoplus_{i=1}^{\ell-1} \mathbb{Z} x_{i}^{\prime} \oplus H_{1}\left(\widetilde{S}^{\prime}\right)$ with the 1-cycles $y_{i}$ and $x_{i}^{\prime}$ depicted above. Using the same method and notations as above, one can compute the Hermitian matrix $H_{S^{\prime}}\left(\omega^{\prime}\right)$ corresponding to the basis $\left\langle y_{1}, \ldots, y_{\ell}, x_{1}^{\prime} \ldots, x_{\ell-1}^{\prime}\right\rangle \cup$ $\mathcal{B}$ of $H_{1}\left(S^{\prime}\right)$. It is given by

$$
\prod_{i=1}^{\mu}\left|1-\omega_{i}\right|^{2}\left(\begin{array}{ccc}
|\lambda|^{2}\left(I_{\ell_{-}} \oplus-I_{\ell_{+}}\right) & \bar{\lambda} M & 0 \\
\lambda M^{T} & \lambda D+\bar{\lambda} D^{T} & \lambda C^{-}+\bar{\lambda} C^{+} \\
0 & \left(\bar{\lambda} C^{-}+\lambda C^{+}\right)^{T} & \prod_{i=1}^{\mu-1}\left|1-\omega_{i}\right|^{-2} H_{\widetilde{S}^{\prime}}\left(\omega^{\prime}\right)
\end{array}\right)
$$


where $I_{k}$ denotes the $(k \times k)$-identity matrix and

$$
M=\left(\begin{array}{rrr}
1 & & \\
-1 & \ddots & \\
& \ddots & 1 \\
& & -1
\end{array}\right) \text {. }
$$

Using the equalities $(\star)$ and $(\star \star)$ together with the fact that $\lambda \neq 0$, it is easy to check that the matrix $H_{S^{\prime}}\left(\omega^{\prime}\right)$ is conjugate to the Hermitian matrix $I_{\ell_{-}} \oplus-I_{\ell_{+}} \oplus$ $H_{S}\left(\omega^{\prime}, \omega_{\mu}\right)$. Hence,

$$
\begin{aligned}
\sigma_{L^{\prime}}\left(\omega^{\prime}\right) & =\operatorname{sign}\left(H_{S^{\prime}}\left(\omega^{\prime}\right)\right)=\operatorname{sign}\left(H_{S}\left(\omega^{\prime}, \omega_{\mu}\right)\right)+\ell_{-}-\ell_{+} \\
& =\sigma_{L}\left(\omega^{\prime}, \omega_{\mu}\right)-l k\left(L_{\mu}, L_{\mu+1}\right),
\end{aligned}
$$

and null $H_{S^{\prime}}\left(\omega^{\prime}\right)=\operatorname{null} H_{S}\left(\omega^{\prime}, \omega_{\mu}\right)$. Since $\beta_{0}(S)=\beta_{0}\left(S^{\prime}\right)$, the proposition is proved.

Note that we shall give an alternative proof of this result in Subsection 6.3.

2.3. Basic properties of $\sigma_{L}$ and $\eta_{L}$. We conclude this section with an enumeration of several properties of the signature and nullity. Deeper properties shall be presented in the following sections. The first proposition follows easily from the fact that $H(\omega)$ is Hermitian.

Proposition 2.7. For any $\left(\omega_{1}, \ldots, \omega_{\mu}\right) \in T_{*}^{\mu}$,

$$
\begin{aligned}
\sigma_{L}\left(\omega_{1}^{-1}, \ldots, \omega_{\mu}^{-1}\right) & =\sigma_{L}\left(\omega_{1}, \ldots, \omega_{\mu}\right), \\
\eta_{L}\left(\omega_{1}^{-1}, \ldots, \omega_{\mu}^{-1}\right) & =\eta_{L}\left(\omega_{1}, \ldots, \omega_{\mu}\right) .
\end{aligned}
$$

Proposition 2.8. Let $L$ be a colored link, and let $L^{\prime}$ be the colored link obtained from $L$ by reversing the orientation of every component of the sublink $L_{1}$ of $L$. Then, for all $\omega=\left(\omega_{1}, \ldots, \omega_{\mu}\right)$ in $T_{*}^{\mu}$,

$$
\begin{aligned}
& \sigma_{L^{\prime}}\left(\omega_{1}, \ldots, \omega_{\mu}\right)=\sigma_{L}\left(\omega_{1}^{-1}, \omega_{2}, \ldots, \omega_{\mu}\right), \\
& \eta_{L^{\prime}}\left(\omega_{1}, \ldots, \omega_{\mu}\right)=\eta_{L}\left(\omega_{1}^{-1}, \omega_{2}, \ldots, \omega_{\mu}\right) .
\end{aligned}
$$

Proof. If $S=S_{1} \cup S_{2} \cup \cdots \cup S_{\mu}$ is a C-complex for $L$, then $S^{\prime}=\left(-S_{1}\right) \cup S_{2} \cup \cdots \cup S_{\mu}$ is a C-complex for $L^{\prime}$. Since $A_{S^{\prime}}^{\varepsilon^{\prime}}=A_{S}^{\varepsilon}$ with $\varepsilon_{1}^{\prime}=-\varepsilon_{1}$ and $\varepsilon_{i}^{\prime}=\varepsilon_{i}$ for $i>1$, the corresponding Hermitian matrices $H^{\prime}$ and $H$ are related by $H^{\prime}\left(\omega_{1}, \omega_{2}, \ldots, \omega_{\mu}\right)=$ $H\left(\omega_{1}^{-1}, \omega_{2}, \ldots, \omega_{\mu}\right)$.

Corollary 2.9. If $-L$ denotes the colored link $L$ with the opposite orientation, then $\sigma_{-L}=\sigma_{L}$ and $\eta_{-L}=\eta_{L}$.

So the signature cannot distinguish between a colored link and its inverse. On the other hand, it is a useful invariant for telling apart a colored link and its mirror image.

Proposition 2.10. If $\bar{L}$ denotes the mirror image of the colored link $L$, then $\sigma_{\bar{L}}=$ $-\sigma_{L}$ and $\eta_{\bar{L}}=\eta_{L}$.

Proof. If $S$ is a C-complex for $L$, then the mirror image $S^{\prime}$ of $S$ is a C-complex for $\bar{L}$. Therefore, $A_{S^{\prime}}^{\varepsilon}=-A_{S}^{\varepsilon}$, and the Hermitian matrices $H$ and $H^{\prime}$ satisfy $H^{\prime}(\omega)=-H(\omega)$. 
Corollary 2.11. If a colored link $L$ is isotopic to its mirror image, then $\sigma_{L}$ is identically zero.

Finally, the signature and nullity behave well under connected and disjoint sums.

Proposition 2.12. Let $L^{\prime}=L_{1} \cup \cdots \cup L_{\nu-1} \cup L_{\nu}^{\prime}$ and $L^{\prime \prime}=L_{\nu}^{\prime \prime} \cup L_{\nu+1} \cup \cdots \cup L_{\mu}$ be two colored links. Consider a colored link $L=L_{1} \cup \cdots \cup L_{\mu}$, where $L_{\nu}$ is a connected sum of $L_{\nu}^{\prime}$ and $L_{\nu}^{\prime \prime}$ along any of their components. Then, for all $\omega_{1}, \ldots, \omega_{\mu} \in S^{1} \backslash\{1\}$,

$$
\begin{aligned}
& \sigma_{L}\left(\omega_{1}, \ldots, \omega_{\mu}\right)=\sigma_{L^{\prime}}\left(\omega_{1}, \ldots, \omega_{\nu}\right)+\sigma_{L^{\prime \prime}}\left(\omega_{\nu}, \ldots, \omega_{\mu}\right), \\
& \eta_{L}\left(\omega_{1}, \ldots, \omega_{\mu}\right)=\eta_{L^{\prime}}\left(\omega_{1}, \ldots, \omega_{\nu}\right)+\eta_{L^{\prime \prime}}\left(\omega_{\nu}, \ldots, \omega_{\mu}\right) .
\end{aligned}
$$

Proof. Given $S^{\prime}$ a C-complex for $L^{\prime}$ and $S^{\prime \prime}$ a C-complex for $L^{\prime \prime}$, a C-complex $S$ for $L$ is given by the band sum of $S^{\prime}$ and $S^{\prime \prime}$ along the corresponding components of $S_{\nu}^{\prime}$ and $S_{\nu}^{\prime \prime}$. Since $S^{\prime}$ and $S^{\prime \prime}$ have no closed components, this band can be chosen such that only its ends meet $S^{\prime}$ and $S^{\prime \prime}$. Clearly, $A_{S}^{\varepsilon}=A_{S^{\prime}}^{\varepsilon^{\prime}} \oplus A_{S^{\prime \prime}}^{\varepsilon^{\prime \prime}}$ with $\varepsilon^{\prime}=\left(\varepsilon_{1}, \ldots, \varepsilon_{\nu}\right)$ and $\varepsilon^{\prime \prime}=\left(\varepsilon_{\nu}, \ldots, \varepsilon_{\mu}\right)$. The corresponding Hermitian matrices $H, H^{\prime}$ and $H^{\prime \prime}$ satisfy

$$
H\left(\omega_{1}, \ldots, \omega_{\mu}\right)=\prod_{i>\nu}\left|1-\omega_{i}\right|^{2} H^{\prime}\left(\omega_{1}, \ldots, \omega_{\nu}\right) \oplus \prod_{i<\nu}\left|1-\omega_{i}\right|^{2} H^{\prime \prime}\left(\omega_{\nu}, \ldots, \omega_{\mu}\right) .
$$

Since $\omega_{i} \neq 1$ for all $i$ and $\beta_{0}(S)=\beta_{0}\left(S^{\prime}\right)+\beta_{0}\left(S^{\prime \prime}\right)-1$, this implies the proposition.

Proposition 2.13. Let $L^{\prime}$ and $L^{\prime \prime}$ be colored links with disjoint sets of colors. Consider the colored link $L$ given by the disjoint sum of $L^{\prime}$ and $L^{\prime \prime}$. Then,

$$
\begin{aligned}
& \sigma_{L}\left(\omega^{\prime}, \omega^{\prime \prime}\right)=\sigma_{L^{\prime}}\left(\omega^{\prime}\right)+\sigma_{L^{\prime \prime}}\left(\omega^{\prime \prime}\right), \\
& \eta_{L}\left(\omega^{\prime}, \omega^{\prime \prime}\right)=\eta_{L^{\prime}}\left(\omega^{\prime}\right)+\eta_{L^{\prime \prime}}\left(\omega^{\prime \prime}\right)+1 .
\end{aligned}
$$

Proof. Let $S^{\prime}$ and $S^{\prime \prime}$ be C-complexes for $L^{\prime}$ and $L^{\prime \prime}$. A C-complex $S$ for $L$ is given by the disjoint union of $S^{\prime}$ and $S^{\prime \prime}$. Clearly, $A_{S}^{\left(\varepsilon^{\prime}, \varepsilon^{\prime \prime}\right)}=A_{S^{\prime}}^{\varepsilon^{\prime}} \oplus A_{S^{\prime \prime}}^{\varepsilon^{\prime \prime}}$, so

$$
H\left(\omega^{\prime}, \omega^{\prime \prime}\right)=\prod_{i}\left|1-\omega_{i}^{\prime \prime}\right|^{2} H^{\prime}\left(\omega^{\prime}\right) \oplus \prod_{j}\left|1-\omega_{j}^{\prime}\right|^{2} H^{\prime \prime}\left(\omega^{\prime \prime}\right) .
$$

Since $\beta_{0}(S)=\beta_{0}\left(S^{\prime}\right)+\beta_{0}\left(S^{\prime \prime}\right)$, the proposition is proved.

\section{The Alexander module of a COlORed link}

Associated to a $\mu$-colored link $L$ with exterior $X$ is a natural $\mathbb{Z}^{\mu}$-covering $\widetilde{X} \rightarrow X$. The aim of this section is to show how the space $\widetilde{X}$ can be constructed from a Ccomplex for $L$. This leads to a presentation of the Alexander module of $L$, that is, the $\mathbb{Z}\left[t_{1}^{ \pm 1}, \ldots, t_{\mu}^{ \pm 1}\right]$-module $H_{1}(\widetilde{X})$. This generalizes a celebrated theorem of Seifert, which corresponds to the case $\mu=1$.

We shall use these results in the next section to derive relations between the signature, the nullity, and the Alexander invariants of colored links.

3.1. Basics. Let $L=L_{1} \cup \cdots \cup L_{\mu}$ be a colored link, and let $X$ denote its exterior. The epimorphism $\pi_{1}(X) \rightarrow \mathbb{Z}^{\mu}$ given by $\gamma \mapsto\left(l k\left(\gamma, L_{1}\right), \ldots, l k\left(\gamma, L_{\mu}\right)\right)$ induces a regular $\mathbb{Z}^{\mu}$-covering $\widetilde{X} \rightarrow X$. The homology of $\widetilde{X}$ is a natural module over the ring $\Lambda_{\mu}=\mathbb{Z}\left[t_{1}^{ \pm 1}, \ldots, t_{\mu}^{ \pm 1}\right]$, where $t_{i}$ denotes the covering transformation corresponding to an oriented meridian of $L_{i}$. The $\Lambda_{\mu}$-module $H_{1}(\tilde{X})$ is called the Alexander module of the colored link $L$. Of course, if $\mu=1$, then $H_{1}(\tilde{X})$ is nothing but the usual Alexander module of the link $L$. 
To extract handy information from such a cumbersome invariant as a module over $\Lambda_{\mu}$, the standard trick is to consider its elementary ideals. Although these objects are widely used, their definitions vary according to the authors. Therefore, we shall now clarify the meaning of these concepts in the present work. Let $R$ be a noetherian factorial domain, that is, an integral domain in which every ideal is finitely generated, and every non-zero non-invertible element has a unique factorization. Let $P$ be an $m \times n$ matrix with coefficients in $R$. Let us denote by $E_{r}(P)$ the ideal of $R$ generated by all the $(m-r) \times(m-r)$ minors of $P$. By convention, $E_{r}(P)=(0)$ if $r<0$ and $E_{r}(P)=R$ if $r \geq m$. Let $\Delta_{r}(P)$ denote the greatest common divisor of the elements of $E_{r}(P)$. (Recall that the greatest common divisor of $a_{1}, \ldots, a_{n}$ in $R$ is an element $d$ of $R$ which divides $a_{i}$ for all $i$, and such that if $c \in R$ divides $a_{i}$ for all $i$, then $c$ divides $d$.) Since $R$ is a noetherian factorial domain, $\Delta_{r}(P)$ exists, and is well-defined up to multiplication by a unit of $R$. Given $\Delta$ and $\Delta^{\prime}$ in $R$, let us note $\Delta \doteq \Delta^{\prime}$ if $\Delta=u \Delta^{\prime}$ for some unit $u$ of $R$. Now, let $M$ be a module over a factorial ring $R$. A finite presentation of $M$ is an exact sequence $F \stackrel{\varphi}{\rightarrow} E \rightarrow M \rightarrow 0$, where $E$ and $F$ are free $R$-modules with finite basis. A matrix of $\varphi$ is a presentation matrix of $M$. The $r^{\text {th }}$ elementary ideal of $M$ is the ideal of $R$ given by $E_{r}(M):=E_{r}(P)$, where $P$ is any presentation matrix of $M$. It is easy to check that these ideals do not depend on the presentation of $M$. In particular, the element $\Delta_{r}(M):=\Delta_{r}(P)$ of $R$ is well defined up to multiplication by a unit of $R$.

Let us now turn back to colored links. Given a $\mu$-colored link $L$, we just defined the Alexander module of $L$ as some module $H_{1}(\widetilde{X})$ over the ring $\Lambda_{\mu}$. The $r^{\text {th }}$ elementary ideal $E_{r}\left(H_{1}(\widetilde{X})\right)$ is the $r^{\text {th }}$ Alexander ideal of $L$, and is denoted by $E_{r}(L)$. The polynomial $\Delta_{r}(L):=\Delta_{r}\left(H_{1}(\widetilde{X})\right)$ is called the $r^{\text {th }}$ Alexander polynomial of $L ; \Delta_{0}(L)$ is called the Alexander polynomial of $L$, and is denoted by $\Delta_{L}$. Again, note that $\Delta_{r}(L)$ is only defined up to multiplication by a unit of $\Lambda_{\mu}$, that is, by $\pm t_{1}^{m_{1}} \cdots t_{\mu}^{m_{\mu}}$ with integers $m_{i}$.

3.2. A presentation of the Alexander module using a C-complex. Fix a colored link $L=L_{1} \cup \cdots \cup L_{\mu}$. Consider a C-complex $S=S_{1} \cup \cdots \cup S_{\mu}$ for $L$ such that each $S_{i}$ is connected and $S_{i} \cap S_{j} \neq \emptyset$ for all $i \neq j$. (Such a C-complex exists by transformations (T1) and (T2) of Lemma 2.2.) For $i=1, \ldots, \mu$, choose some interior point $v_{i}$ of $S_{i} \backslash \bigcup_{j \neq i} S_{i} \cap S_{j}$. Given a clasp in $S_{i} \cap S_{j}$ with $i<j$, consider an oriented edge in $S_{i} \cup S_{j}$ joining $v_{i}$ and $v_{j}$ and passing through this single clasp as described in Figure 2, This leads to a collection of oriented edges $\left\{e_{i j}^{1}, \ldots, e_{i j}^{c(i, j)}\right\}$, where $c(i, j)$ denotes the number of clasps in $S_{i} \cap S_{j}$ (that is: the number of connected components of $S_{i} \cap S_{j}$ ). Let $K_{i j} \subset S_{i} \cup S_{j}$ denote the graph given by the union of these edges. Finally let $K_{\mu}$ be the complete graph with vertices $\left\{v_{i}\right\}_{1 \leq i \leq \mu}$ and edges $\left\{e_{i j}^{1}\right\}_{1 \leq i<j \leq \mu}$.

Lemma 3.1. The homology of $S=S_{1} \cup \cdots \cup S_{\mu}$ is equal to

$$
H_{1}(S)=\bigoplus_{1 \leq i \leq \mu} H_{1}\left(S_{i}\right) \oplus \bigoplus_{1 \leq i<j \leq \mu} H_{1}\left(K_{i j}\right) \oplus H_{1}\left(K_{\mu}\right) .
$$

Furthermore, a basis of $H_{1}\left(K_{i j}\right)$ is given by $\left\langle\beta_{i j}^{\ell}\right\rangle_{1 \leq \ell \leq c(i, j)-1}$, where $\beta_{i j}^{\ell}=e_{i j}^{\ell}-e_{i j}^{\ell+1}$. Finally, a basis of $H_{1}\left(K_{\mu}\right)$ is given by $\left\langle\gamma_{1 i j}\right\rangle_{2 \leq i<j \leq \mu}$, where $\gamma_{i j k}=e_{i j}^{1}-e_{k i}^{1}+e_{j k}^{1}$. 
Proof. The C-complex $S$ can be constructed as follows. Consider the complete graph $K_{\mu}$. Add the graphs $K_{i j}$ one by one, for $1 \leq i<j \leq \mu$. Finally, paste $S_{1}, S_{2}, \ldots, S_{\mu}$. Note that at each step, the pasting is done along a contractible space. A recursive use of the Mayer-Vietoris exact sequence therefore leads to the first statement of the lemma. The fact that $H_{1}\left(K_{i j}\right)=\bigoplus_{\ell=1}^{c(i, j)-1} \mathbb{Z} \beta_{i j}^{\ell}$ is clear. Finally, we have the relation $\gamma_{i j k}=\gamma_{1 j k}-\gamma_{1 i k}+\gamma_{1 i j}$. Hence the family $\left\langle\gamma_{1 i j}\right\rangle_{2 \leq i<j \leq \mu}$ generates $H_{1}\left(K_{\mu}\right)$. Furthermore, $1-\operatorname{rk} H_{1}\left(K_{\mu}\right)=\chi\left(K_{\mu}\right)=\mu-\left(\begin{array}{c}\mu \\ 2\end{array}\right)$, so rk $H_{1}\left(K_{\mu}\right)=$ $\left(\begin{array}{c}\mu-1 \\ 2\end{array}\right)$, and this family is a basis of $H_{1}\left(K_{\mu}\right)$.

Recall the homomorphism $i^{\varepsilon}: H_{1}(S) \rightarrow H_{1}\left(S^{3} \backslash S\right)$ of Subsection 2.1.

Theorem 3.2. Let $L=L_{1} \cup \cdots \cup L_{\mu}$ be a colored link, and consider a C-complex $S=S_{1} \cup \cdots \cup S_{\mu}$ for $L$ such that each $S_{i}$ is connected and $S_{i} \cap S_{j} \neq \emptyset$ for all $i \neq j$. Let $\alpha: H_{1}(S) \otimes_{\mathbb{Z}} \Lambda_{\mu} \rightarrow H_{1}\left(S^{3} \backslash S\right) \otimes_{\mathbb{Z}} \Lambda_{\mu}$ be the homomorphism of $\Lambda_{\mu}$-modules given by

$$
\alpha=\sum_{\varepsilon} \varepsilon_{1} \cdots \varepsilon_{\mu} t_{1}^{\frac{\varepsilon_{1}+1}{2}} \cdots t_{\mu}^{\frac{\varepsilon_{\mu}+1}{2}} i^{\varepsilon},
$$

where the sum is on all sequences $\varepsilon=\left(\varepsilon_{1}, \ldots, \varepsilon_{\mu}\right)$ of \pm 1 's. Then, the Alexander module $H_{1}(\widetilde{X})$ of $L$ admits the finite presentation

$$
\widehat{H} \otimes_{\mathbb{Z}} \Lambda_{\mu} \stackrel{\widehat{\alpha}}{\longrightarrow} H_{1}\left(S^{3} \backslash S\right) \otimes_{\mathbb{Z}} \Lambda_{\mu} \longrightarrow H_{1}(\tilde{X}) \longrightarrow 0,
$$

where $\widehat{H}=\bigoplus_{1 \leq i \leq \mu} H_{1}\left(S_{i}\right) \oplus \bigoplus_{1 \leq i<j \leq \mu} H_{1}\left(K_{i j}\right) \oplus \bigoplus_{1 \leq i<j<k \leq \mu} \mathbb{Z} \gamma_{i j k}$ and $\widehat{\alpha}$ is given by

- $\widehat{\alpha}=\prod_{n \neq i}\left(t_{n}-1\right)^{-1} \alpha$ on $H_{1}\left(S_{i}\right)$ for $1 \leq i \leq \mu$;

- $\widehat{\alpha}=\prod_{n \neq i, j}\left(t_{n}-1\right)^{-1} \alpha$ on $H_{1}\left(K_{i j}\right)$ for $1 \leq i<j \leq \mu$;

- $\widehat{\alpha}\left(\gamma_{i j k}\right)=\prod_{n \neq i, j, k}\left(t_{n}-1\right)^{-1} \alpha\left(\gamma_{i j k}\right)$ for $1 \leq i<j<k \leq \mu$.

We postpone the proof of this theorem to the end of the section.

For colored links with 1, 2 or 3 colors, this result provides a square presentation matrix of the Alexander module expressed in terms of the Seifert matrices $A^{\varepsilon}$. More precisely, we have the following corollaries.

Corollary 3.3 (Seifert [30]). Let A be a Seifert matrix for the link L. Then, $t A-A^{T}$ is a presentation matrix of the Alexander module of $L$.

Proof. Theorem 3.2 gives the finite presentation

$$
H_{1}(S) \otimes_{\mathbb{Z}} \Lambda \stackrel{\widehat{\alpha}}{\rightarrow} H_{1}\left(S^{3} \backslash S\right) \otimes_{\mathbb{Z}} \Lambda \rightarrow H_{1}(\widetilde{X}) \rightarrow 0,
$$

where $\widehat{\alpha}=\alpha=t i^{+}-i^{-}$. By Alexander duality, a matrix of $i^{+}$(resp. $i^{-}$) is given by the transpose of $A^{+}$(resp. $A^{-}$). Therefore, $t A-A^{T}$ is a presentation matrix.

Similarly, we get the following result.

Corollary 3.4 (Cooper [7). Let $S=S_{1} \cup S_{2}$ be a $C$-complex for a colored link $L=$ $L_{1} \cup L_{2}$. Let $A$ (resp. B) be a matrix of the form $\alpha^{--}$(resp. $\alpha^{-+}$) with respect to a basis of $H_{1}(S)$ adapted to the decomposition $H_{1}(S)=H_{1}\left(S_{1}\right) \oplus H_{1}\left(S_{2}\right) \oplus H_{1}\left(K_{12}\right)$. Then, a presentation matrix of the Alexander module of $L$ is given by

$$
\left(t_{1} t_{2} A-t_{1} B-t_{2} B^{T}+A^{T}\right) \cdot D,
$$



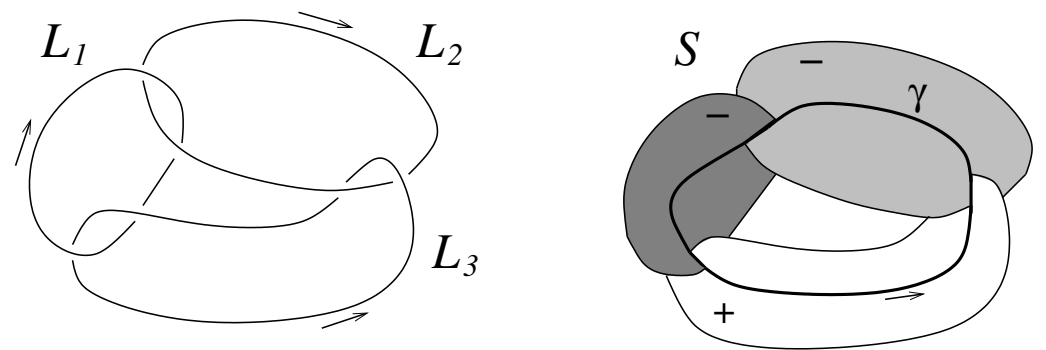

Figure 11. The 3-colored link of Example 3.5.

where $D=\left(D_{i j}\right)$ is the diagonal matrix given by

$$
D_{i i}= \begin{cases}\left(t_{2}-1\right)^{-1} & \text { if } 1 \leq i \leq \beta_{1}\left(S_{1}\right), \\ \left(t_{1}-1\right)^{-1} & \text { if } \beta_{1}\left(S_{1}\right)<i \leq \beta_{1}\left(S_{1}\right)+\beta_{1}\left(S_{2}\right), \\ 1 & \text { if } \beta_{1}\left(S_{1}\right)+\beta_{1}\left(S_{2}\right)<i \leq \beta_{1}(S),\end{cases}
$$

and $\beta_{1}(\cdot)$ denotes the first Betti number.

The case $\mu=3$ is similar, but the complete statement is a little cumbersome. Instead, let us give an example of such a computation.

Example 3.5. Consider the 3-colored link $L$ given in Figure 11, A C-complex $S$ for $L$ is also drawn. Clearly, $H_{1}(S)=\mathbb{Z} \gamma$. Furthermore, $l k\left(\gamma^{\varepsilon}, \gamma\right)=1$ if $\varepsilon_{1}=\varepsilon_{2}=\varepsilon_{3}$, and all the other linking numbers are zero. Hence, a matrix of $\widehat{\alpha}=\alpha$ is given by $\left(t_{1} t_{2} t_{3}-1\right)$, and $H_{1}(\widetilde{X})=\Lambda_{3} /\left(t_{1} t_{2} t_{3}-1\right)$.

For colored links with $\mu \geq 4$ colors, the presentation of Theorem 3.2 has deficiency $\left(\begin{array}{c}\mu-1 \\ 3\end{array}\right)$, so the corresponding presentation matrix is not square. This is not a surprise. Indeed, Crowell and Strauss [8] proved that if an ordered link has $\mu \geq 4$ components and if $\Delta_{L} \neq 0$, then its Alexander module does not admit any square presentation matrix. Their proof applies to colored links as well. Therefore, it is not possible to get a presentation matrix of $H_{1}(\widetilde{X})$ using the Seifert matrices $A^{\varepsilon}$ if $\mu>3$. Nevertheless, it is possible to compute the Alexander invariants up to some indeterminacy. More precisely, let $\Lambda_{\mu}^{\prime}$ denote the localization of the ring $\Lambda_{\mu}$ with respect to the multiplicative system generated by $\left\{t_{i}-1\right\}_{1 \leq i \leq \mu}$.

Corollary 3.6. Let $L$ be a $\mu$-colored link. Consider a $C$-complex $S$ for $L$ such that $S_{i}$ is connected for all $i$ and $S_{i} \cap S_{j} \neq \emptyset$ for all $i \neq j$. Then the corresponding matrix

$$
A\left(t_{1}, \ldots, t_{\mu}\right)=\sum_{\varepsilon} \varepsilon_{1} \cdots \varepsilon_{\mu} t_{1}^{\frac{1-\varepsilon_{1}}{2}} \cdots t_{\mu}^{\frac{1-\varepsilon_{\mu}}{2}} A^{\varepsilon}
$$

is a presentation matrix of the $\Lambda_{\mu}^{\prime}$-module $H_{1}(\tilde{X}) \otimes_{\Lambda_{\mu}} \Lambda_{\mu}^{\prime}$. In particular, for all $r$, there are non-negative integers $m_{i}$ such that the following equality holds in $\Lambda_{\mu}$ :

$$
\prod_{i=1}^{\mu}\left(1-t_{i}\right)^{m_{i}} \Delta_{r}(L) \doteq \Delta_{r}\left(A\left(t_{1}, \ldots, t_{\mu}\right)\right) .
$$

Proof. Let $\mathcal{B}$ be a basis of $H_{1}(S)$ adapted to the decomposition given in Lemma 3.1. Consider the dual basis $\mathcal{B}^{*}$ of $H_{1}\left(S^{3} \backslash S\right)$ obtained by Alexander duality. Clearly, the matrix of $i^{\varepsilon}: H_{1}(S) \rightarrow H_{1}\left(S^{3} \backslash S\right)$ with respect to the bases $\mathcal{B}, \mathcal{B}^{*}$ is 

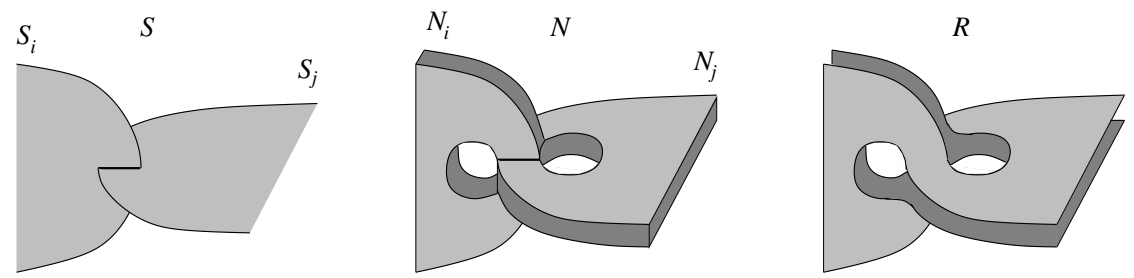

Figure 12. The C-complex $S$ and the spaces $N$ and $R$ near a clasp.

the transposed matrix of $\alpha^{\varepsilon}: H_{1}(S) \times H_{1}(S) \rightarrow \mathbb{Z}$ with respect to $\mathcal{B}$, that is, a Seifert matrix $A^{-\varepsilon}$. Consider the basis $\widehat{\mathcal{B}}=\mathcal{B} \cup\left\langle\gamma_{i j k}\right\rangle_{2 \leq i<j<k \leq \mu}$ of $\widehat{H}$, and the extension $\widehat{i}^{\varepsilon}: \widehat{H} \rightarrow H_{1}\left(S^{3} \backslash S\right)$ of $i^{\varepsilon}$. The matrix $\widehat{A}^{-\varepsilon}$ of $\widehat{i}^{\varepsilon}$ with respect to the bases $\widehat{\mathcal{B}}, \mathcal{B}^{*}$ is a matrix with $\beta_{1}(S)$ rows and $\beta_{1}(S)+\left(\begin{array}{c}\mu-1 \\ 3\end{array}\right)$ columns. $A^{-\varepsilon}$ is made of the first $\beta_{1}(S)$ columns of $\widehat{A}^{-\varepsilon}$. Set

$$
B=\sum_{\varepsilon} \varepsilon_{1} \cdots \varepsilon_{\mu} t_{1}^{\frac{\varepsilon_{1}+1}{2}} \cdots t_{\mu}^{\frac{\varepsilon_{\mu}+1}{2}} A^{-\varepsilon} \quad \text { and } \quad \widehat{B}=\sum_{\varepsilon} \varepsilon_{1} \cdots \varepsilon_{\mu} t_{1}^{\frac{\varepsilon_{1}+1}{2}} \cdots t_{\mu}^{\frac{\varepsilon_{\mu}+1}{2}} \widehat{A}^{-\varepsilon} \text {. }
$$

By Theorem 3.2 a presentation matrix of the $\Lambda_{\mu}$-module $H_{1}(\widetilde{X})$ is given by $P=$ $\widehat{B} D^{-1}$, where $D$ is a diagonal matrix whose diagonal entries are products of factors of the form $t_{i}-1$. Therefore, $\widehat{B}$ is a presentation matrix of the $\Lambda_{\mu}^{\prime}$-module $H_{1}(\tilde{X}) \otimes_{\Lambda_{\mu}} \Lambda_{\mu}^{\prime}$. Now, let $\ell_{i j k}$ denote the column in $\widehat{B}$ corresponding to the element $\gamma_{i j k}$ of $\widehat{\mathcal{B}}$, for $1 \leq i<j<k \leq \mu$. If $i>1$, then $\gamma_{i j k}=\gamma_{1 i j}-\gamma_{1 i k}+\gamma_{1 j k}$ in $H_{1}(S)$. Therefore, $\ell_{i j k}=\ell_{1 i j}-\ell_{1 i k}+\ell_{1 j k}$ for all $2 \leq i<j<k \leq \mu$. So the $\left(\begin{array}{c}\mu-1 \\ 3\end{array}\right)$ last columns of $\widehat{B}$ are linear combinations of the other columns. Hence, $B$ is also a presentation matrix of $H_{1}(\widetilde{X}) \otimes_{\Lambda_{\mu}} \Lambda_{\mu}^{\prime}$. Since $B=(-1)^{\mu} A\left(t_{1}, \ldots, t_{\mu}\right)$, the corollary is proved.

Using this result, it is possible to give an intrinsic interpretation of the nullity $\eta_{L}(\omega)$ for $\omega$ with rational coordinates. Indeed, consider the character $\chi_{\omega}: H_{1}(X) \rightarrow$ $\mathbb{C}^{*}$ which maps a meridian of $L_{i}$ to $\omega_{i}$. If $\omega$ has rational coordinates, then the image of $\chi_{\omega}$ is a finite cyclic group $C_{q}$. The homology of the corresponding cyclic covering $X^{q} \rightarrow X$ is a module over $\mathbb{Z}\left[C_{q}\right]$, and so is $\mathbb{C}$ via the inclusion $C_{q} \subset \mathbb{C}^{*}$. Then,

$$
\eta_{L}(\omega)=\operatorname{dim}_{\mathbb{C}} H_{1}\left(X^{q}\right) \otimes_{\mathbb{Z}\left[C_{q}\right]} \mathbb{C} .
$$

We shall give a 4-dimensional proof of this result in Section 6.

3.3. Proof of Theorem 3.2. As in Section 2, let $X$ denote the exterior of $L$, and let $N_{i}=S_{i} \times[-1,1]$ be a closed bicollar neighbourhood of $S_{i}$. Set $N=$ $X \cap \bigcup_{i=1}^{\mu} N_{i}, Y=X \backslash \bigcup_{i=1}^{\mu}$ int $N_{i}$ and $R=N \cap Y$. (See Figure 12 for an illustration of these spaces near a clasp.) Recall that the regular $\mathbb{Z}^{\mu}$-covering $\widetilde{X} \stackrel{p}{\rightarrow} X$ is induced by the epimorphism $\pi_{1}(X) \rightarrow \mathbb{Z}^{\mu}$ given by $\gamma \mapsto\left(l k\left(\gamma, L_{1}\right), \ldots, l k\left(\gamma, L_{\mu}\right)\right)$. The decomposition $X=N \cup Y$ leads to a Mayer-Vietoris exact sequence of $\Lambda_{\mu}$-modules

$$
H_{1}(\widetilde{R}) \stackrel{(\varphi,-\psi)}{\longrightarrow} H_{1}(\widetilde{N}) \oplus H_{1}(\tilde{Y}) \longrightarrow H_{1}(\tilde{X}) \longrightarrow H_{0}(\widetilde{R}) \stackrel{\left(\varphi_{0},-\psi_{0}\right)}{\longrightarrow} H_{0}(\tilde{N}) \oplus H_{0}(\tilde{Y})
$$

where $\widetilde{R}$ (resp. $\widetilde{N}, \widetilde{Y})$ stands for $p^{-1}(R)\left(\operatorname{resp} . p^{-1}(N), p^{-1}(Y)\right)$, and $\varphi, \psi, \varphi_{0}, \psi_{0}$ are inclusion homomorphisms. Note that $R \cap S=\emptyset$. Therefore, given any loop $\gamma$ in $R, l k\left(\gamma, L_{i}\right)=\gamma \cdot S_{i}=0$ for $1 \leq i \leq \mu$. Hence, $\widetilde{R} \stackrel{p}{\rightarrow} R$ is the trivial $\mathbb{Z}^{\mu}$-covering 


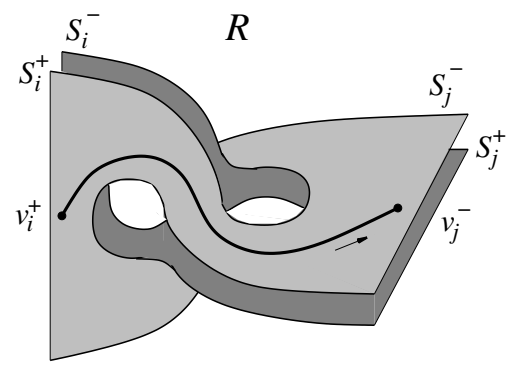

FiguRE 13.

and $H_{*}(\widetilde{R})$ is isomorphic to $H_{*}(R) \otimes \Lambda$, where $\otimes=\otimes_{\mathbb{Z}}$ and $\Lambda=\Lambda_{\mu}$. For the same reason, we can identify $H_{*}(\widetilde{Y})$ with $H_{*}(Y) \otimes \Lambda$.

We now claim that the homomorphism $\left(\varphi_{0},-\psi_{0}\right)$ is injective. Indeed, $S$ is connected, so $R$ is connected as well, provided $\mu>1$. In this case, $\psi_{0}=i d_{\Lambda}$ and $\left(\varphi_{0},-\psi_{0}\right)$ is injective. If $\mu=1$, then $H_{0}(R) \otimes \Lambda=\Lambda \oplus \Lambda$ and $H_{0}(\widetilde{N})=\Lambda=$ $H_{0}(Y) \otimes \Lambda$. The matrix of $\left(\varphi_{0},-\psi_{0}\right)$ with respect to some well-chosen bases is equal to $\left(\begin{array}{rr}1 & t \\ -1 & -1\end{array}\right)$, so the homomorphism is injective. By this claim, we get the exact sequence

$$
H_{1}(R) \otimes \Lambda \stackrel{(\varphi,-\psi)}{\longrightarrow} H_{1}(\widetilde{N}) \oplus\left(H_{1}(Y) \otimes \Lambda\right) \longrightarrow H_{1}(\widetilde{X}) \rightarrow 0 .
$$

Let us assume momentarily that the inclusion homomorphism $\varphi$ is onto. In this case, the following sequence is exact:

$$
\operatorname{ker} \varphi \stackrel{\psi}{\longrightarrow} H_{1}(Y) \otimes \Lambda \longrightarrow H_{1}(\tilde{X}) \longrightarrow 0 .
$$

Since $H_{1}(Y)=H_{1}\left(S^{3} \backslash S\right)$, we are left with the computation of the homomorphism $\varphi: H_{1}(R) \otimes \Lambda \rightarrow H_{1}(\widetilde{N})$. We shall divide this tedious computation into several steps.

Computation of $H_{1}(R)$. Let $v_{i}$ be an interior point of $\left(S_{i} \cap X\right) \backslash \bigcap_{j \neq i} S_{i} \cap S_{j}$. For $\varepsilon_{i}= \pm 1$, set $S_{i}^{\varepsilon_{i}}=S_{i} \times\left\{\varepsilon_{i}\right\} \subset S_{i} \times[-1,1]=N_{i}$, and let $v_{i}^{\varepsilon_{i}} \in R$ denote the point $v_{i} \times\left\{\varepsilon_{i}\right\} \in N_{i}$. Fix a clasp in $S_{i} \cap S_{j}$ with $i<j$ and two signs $\varepsilon_{i}, \varepsilon_{j}$. Consider an oriented edge in $R$ joining $v_{i}^{\varepsilon_{i}}$ to $v_{j}^{\varepsilon_{j}}$, and passing near this single clasp (see Figure 13).

This leads to a collection $\mathcal{E}_{i j}^{\varepsilon_{i} \varepsilon_{j}}$ of $c(i, j)$ oriented edges, where $c(i, j)$ is the number of clasps in $S_{i} \cap S_{j}$. Let $K_{i j}^{\varepsilon_{i} \varepsilon_{j}} \subset R$ denote the graph given by the union of these edges. Finally, let $K_{\mu}^{ \pm}$denote the graph with vertices $\left\{v_{i}^{\varepsilon_{i}} ; 1 \leq i \leq \mu, \varepsilon_{i}= \pm 1\right\}$ and edges $\left\{e_{i j}^{\varepsilon_{i} \varepsilon_{i}} ; 1 \leq i<j \leq \mu, \varepsilon_{i}, \varepsilon_{j}= \pm 1\right\}$, where $e_{i j}^{\varepsilon_{i} \varepsilon_{i}}$ is one distinguished element of $\mathcal{E}_{i j}^{\varepsilon_{i} \varepsilon_{j}}$. As in the proof of Lemma 3.1, observe that $R$ can be constructed as follows. Consider the graph $K_{\mu}^{ \pm}$. Add the graphs $K_{i j}^{--}$one by one, for $1 \leq i<j \leq \mu$, and then the graphs $K_{i j}^{-+}, K_{i j}^{+-}$and $K_{i j}^{++}$. Finally, paste $S_{i}^{-}$and $S_{i}^{+}$for $1 \leq i \leq \mu$. At 


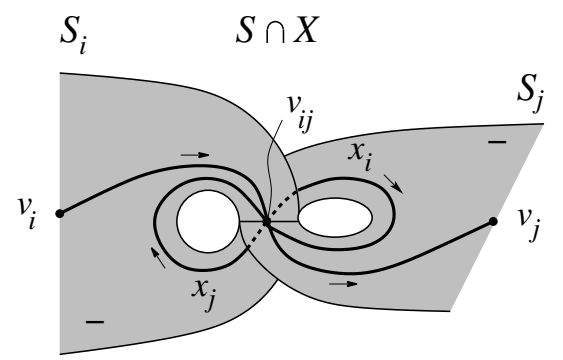

FiguRe 14.

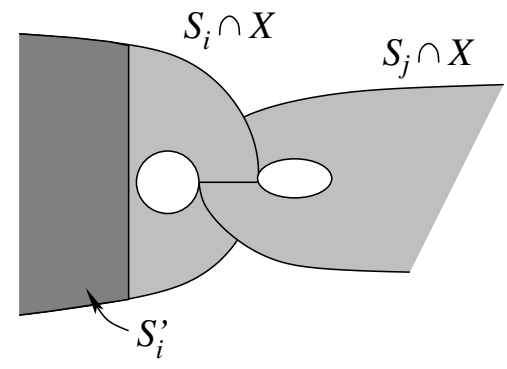

FiguRE 15.

each step, the pasting is done along a contractible space. Therefore,

$$
\begin{aligned}
H_{1}(R) & =\bigoplus_{i}\left(H_{1}\left(S_{i}^{-}\right) \oplus H_{1}\left(S_{i}^{+}\right)\right) \\
& \oplus \bigoplus_{i<j}\left(H_{1}\left(K_{i j}^{--}\right) \oplus H_{1}\left(K_{i j}^{-+}\right) \oplus H_{1}\left(K_{i j}^{+-}\right) \oplus H_{1}\left(K_{i j}^{++}\right)\right) \\
& \oplus H_{1}\left(K_{\mu}^{ \pm}\right) .
\end{aligned}
$$

Computation of $H_{1}(\widetilde{N})$. Consider the complete oriented graph $K_{\mu}$ defined above Lemma 3.1. Add a vertex $v_{i j}$ in the interior of the edge joining $v_{i}$ with $v_{j}$, and paste two oriented loops $x_{i}$ and $x_{j}$ based at $v_{i j}$. The resulting graph, denoted by $\Gamma_{\mu}$, is naturally embedded in $S \cap X \subset N$ as illustrated in Figure 14. Similarly, let $\Gamma_{i j} \subset S_{i} \cap S_{j} \cap X$ be the oriented graph obtained from $K_{i j} \subset S_{i} \cap S_{j}$ by adding a vertex in the interior of each edge of $K_{i j}$, and two oriented loops at this vertex. For $i<j$, consider the loop at $v_{i j}$ given by $x_{i} x_{j} x_{i}^{-1} x_{j}^{-1}$. This loop lifts to a loop $\gamma_{i j}$ in $\widetilde{\Gamma}_{i j}:=p^{-1}\left(\Gamma_{i j}\right)$ and to a loop $\gamma_{i j}^{\prime}$ in $\widetilde{\Gamma}_{\mu}:=p^{-1}\left(\Gamma_{\mu}\right)$. We claim that $H_{1}(\widetilde{N})$ splits as follows:

$$
H_{1}(\tilde{N})=\bigoplus_{i}\left(H_{1}\left(S_{i}\right) \otimes \Lambda\right) \oplus\left(\bigoplus_{i<j} H_{1}\left(\widetilde{\Gamma}_{i j}\right) \oplus H_{1}\left(\widetilde{\Gamma}_{\mu}\right)\right) /\left(\gamma_{i j}=\gamma_{i j}^{\prime}\right)_{i<j} .
$$

Indeed, $N$ has the homotopy type of $S \cap X$, which can be contructed as follows. Consider the graph $\Gamma_{\mu}$. Add the graphs $\Gamma_{i j}$ for $1 \leq i<j \leq \mu$, and then the surfaces $S_{i}^{\prime}$ for $1 \leq i \leq \mu$, where $S_{i}^{\prime}$ is obtained from $S_{i} \cap X$ by removing an open neighbourhood of the clasps as illustrated in Figure 15 .

Hence, $\widetilde{N}$ has the homotopy type of $\widetilde{\Gamma}_{\mu} \cup \bigsqcup_{i<j} \widetilde{\Gamma}_{i j} \cup \bigsqcup_{i} p^{-1}\left(S_{i}^{\prime}\right)$. The first union $\Gamma_{\mu} \cup \Gamma_{12}$ is made along $\Gamma_{\mu} \cap \Gamma_{12}$ which has the homotopy type of the wedge of the 
circles $x_{1}$ and $x_{2}$. This yields a Mayer-Vietoris exact sequence of $\Lambda$-modules

$$
0 \rightarrow \Lambda \gamma_{12} \rightarrow H_{1}\left(\widetilde{\Gamma}_{\mu}\right) \oplus H_{1}\left(\widetilde{\Gamma}_{12}\right) \rightarrow H_{1}\left(\widetilde{\Gamma}_{\mu} \cup \widetilde{\Gamma}_{12}\right) \rightarrow \Lambda / \mathcal{I}_{12} \rightarrow \Lambda / \mathcal{I} \oplus \Lambda / \mathcal{I}_{12},
$$

where $\mathcal{I}$ is the augmentation ideal $\left(t_{1}-1, \ldots, t_{\mu}-1\right)$ and $\mathcal{I}_{12}$ the ideal $\left(t_{1}-1, t_{2}-1\right)$ of $\Lambda$. Hence $H_{1}\left(\widetilde{\Gamma}_{\mu} \cup \widetilde{\Gamma}_{12}\right)=\left(H_{1}\left(\widetilde{\Gamma}_{\mu}\right) \oplus H_{1}\left(\widetilde{\Gamma}_{12}\right)\right) /\left(\gamma_{12}=\gamma_{12}^{\prime}\right)$. Using this argument inductively, we get

$$
H_{1}\left(\widetilde{\Gamma}_{\mu} \cup \bigsqcup_{i<j} \widetilde{\Gamma}_{i j}\right)=\left(\bigoplus_{i<j} H_{1}\left(\widetilde{\Gamma}_{i j}\right) \oplus H_{1}\left(\widetilde{\Gamma}_{\mu}\right)\right) /\left(\gamma_{i j}=\gamma_{i j}^{\prime}\right)_{i<j} .
$$

The pasting of $S_{i}^{\prime}$ is performed along a contractible space. Since $p^{-1}\left(S_{i}^{\prime}\right)=S_{i}^{\prime} \times \mathbb{Z}^{\mu}$ and $S_{i}^{\prime}$ has the homotopy type of $S_{i}$, the claim follows from yet another application of the Mayer-Vietoris sequence.

Using the computation of $H_{1}(R)$ and $H_{1}(\tilde{N})$ above, we see that the inclusion homomorphism $\varphi: H_{1}(R) \otimes \Lambda \rightarrow H_{1}(\tilde{N})$ splits into a direct sum $\varphi=\bigoplus_{i} \varphi_{i} \oplus$ $\bigoplus_{i<j} \varphi_{i j} \oplus \varphi_{K}$. We shall now check that each homomorphism in this sum is onto, and then compute its kernel. Each kernel will translate into a family of relations via the inclusion homomorphism $\psi: H_{1}(R) \otimes \Lambda \rightarrow H_{1}\left(S^{3} \backslash S\right) \otimes \Lambda$. Note that up to multiplication by a unit of $\Lambda$, the latter homomorphism is given by $\psi=\psi^{\prime} \otimes i d_{\Lambda}$, where $\psi^{\prime}: H_{1}(R) \rightarrow H_{1}\left(S^{3} \backslash S\right)$ is the inclusion homomorphism.

Computation of $\varphi_{i}$. The homomorphism

$$
\varphi_{i}:\left(H_{1}\left(S_{i}^{-}\right) \oplus H_{1}\left(S_{i}^{+}\right)\right) \otimes \Lambda \rightarrow H_{1}\left(S_{i}\right) \otimes \Lambda
$$

is given by $\varphi_{i}\left(x^{-}, y^{+}\right)=t_{i} x+y$ for $x, y \in H_{1}\left(S_{i}\right)$. Therefore, the homomorphism $\varphi_{i}$ is onto, and its kernel is generated by $\left\{\left(-x^{-}, t_{i} x^{+}\right) ; x \in H_{1}\left(S_{i}\right)\right\}$ as a $\Lambda$-submodule of $\left(H_{1}\left(S_{i}^{-}\right) \oplus H_{1}\left(S_{i}^{+}\right)\right) \otimes \Lambda$. Given $x \in H_{1}\left(S_{i}\right) \subset H_{1}(S)$, we have $\psi\left(x^{+}\right)=i^{+}(x)$ and $\psi\left(x^{-}\right)=i^{-}(x)$, where $i^{+}$(resp. $i^{-}$) is the homomorphism $i^{\varepsilon}$ with $\varepsilon$ any sequence of \pm 1 's such that $\varepsilon_{i}=+1$ (resp. $\varepsilon_{i}=-1$ ). Therefore, $\psi\left(\operatorname{ker}\left(\varphi_{i}\right)\right)=\left(t_{i} i^{+}-i^{-}\right)\left(H_{1}\left(S_{i}\right)\right)$. The restriction to $H_{1}\left(S_{i}\right)$ of the homomorphism $i^{\varepsilon}$ only depends on $\varepsilon_{i}$. Hence, the restriction to $H_{1}\left(S_{i}\right)$ of the homomorphism $\alpha=\sum_{\varepsilon} \varepsilon_{1} \cdots \varepsilon_{\mu} t_{1}^{\frac{\varepsilon_{1}+1}{2}} \cdots t_{\mu}^{\frac{\varepsilon_{\mu}+1}{2}} i^{\varepsilon}$ is equal to $\left(t_{i} i^{+}-i^{-}\right) \prod_{n \neq i}\left(t_{n}-1\right)$. This gives the first set of relations stated in the theorem.

Computation of $\varphi_{i j}$. Let us now consider the inclusion homomorphism

$$
\varphi_{i j}:\left(H_{1}\left(K_{i j}^{--}\right) \oplus H_{1}\left(K_{i j}^{-+}\right) \oplus H_{1}\left(K_{i j}^{+-}\right) \oplus H_{1}\left(K_{i j}^{++}\right)\right) \otimes \Lambda \rightarrow H_{1}\left(\widetilde{\Gamma}_{i j}\right) /\left(\gamma_{i j}\right) .
$$

We need to compute the homology of $\widetilde{\Gamma}_{i j}$. Number the clasps of $S_{i} \cap S_{j}$ from 1 to $c(i, j)$. For $1 \leq \ell<c(i, j)$, consider the loop in $\Gamma_{i j}$ starting at $v_{i}$, going to the vertex corresponding to the clasp number $\ell$, then around $x_{i}$, back to $v_{i}$, to the vertex corresponding to the clasp number $\ell+1$, around $x_{i}^{-1}$ and back to $v_{i}$. This loop lifts to a loop $\delta_{i}^{\ell}$ in $\widetilde{\Gamma}_{i j}$. One checks by induction on $c(i, j) \geq 1$ that

$$
H_{1}\left(\widetilde{\Gamma}_{i j}\right)=\left(H_{1}\left(K_{i j}\right) \otimes \Lambda\right) \oplus \bigoplus_{\ell=1}^{c(i, j)-1}\left(\Lambda \delta_{i}^{\ell} \oplus \Lambda \delta_{j}^{\ell}\right) \oplus \Lambda \gamma_{i j} .
$$

Recall the basis $\left\{\beta_{i j}^{\ell}\right\}_{\ell}$ of $H_{1}\left(K_{i j}\right)$ given in Lemma 3.1. This yields a basis $\left\{\left(\beta_{i j}^{\ell}\right)^{\varepsilon_{i} \varepsilon_{j}}\right\}_{\ell}$ of $H_{1}\left(K_{i j}^{\varepsilon_{i} \varepsilon_{j}}\right)$ for $\varepsilon_{i}, \varepsilon_{j}= \pm 1$. The homomorphism $\varphi_{i j}$ is given by

$$
\begin{array}{ll}
\left(\beta_{i j}^{\ell}\right)^{--} \mapsto t_{i} t_{j} \beta_{i j}^{\ell}, & \left(\beta_{i j}^{\ell}\right)^{++} \mapsto\left(t_{i}+t_{j}-1\right) \beta_{i j}^{\ell}+\delta_{i}^{\ell}-\delta_{j}^{\ell}, \\
\left(\beta_{i j}^{\ell}\right)^{-+} \mapsto t_{i}\left(t_{j} \beta_{i j}^{\ell}-\delta_{j}^{\ell}\right), & \left(\beta_{i j}^{\ell}\right)^{+-} \mapsto t_{j}\left(t_{i} \beta_{i j}^{\ell}+\delta_{i}^{\ell}\right) .
\end{array}
$$


Therefore, $\varphi_{i j}$ is onto and its kernel is generated by

$$
\left\{\left(t_{i} t_{j} \beta^{++},-t_{i} \beta^{+-},-t_{j} \beta^{-+}, \beta^{--}\right) ; \beta \in H_{1}\left(K_{i j}\right)\right\} \text {. }
$$

For $\beta \in H_{1}\left(K_{i j}\right) \subset H_{1}(S), \psi\left(\beta^{++}\right)=i^{++}(\beta)$ where $i^{++}=i^{\varepsilon}$ with $\varepsilon$ any sequence of \pm 1 's such that $\varepsilon_{i}=\varepsilon_{j}=+1$. Similarly, $\psi\left(\beta^{+-}\right)=i^{+-}(\beta), \psi\left(\beta^{-+}\right)=i^{-+}(\beta)$ and $\psi\left(\beta^{--}\right)=i^{--}(\beta)$. Hence, the image of $\operatorname{ker}\left(\varphi_{i j}\right)$ under $\psi$ is the image of $H_{1}\left(K_{i j}\right)$ under the homomorphism $t_{i} t_{j} i^{++}-t_{i} i^{+-}-t_{j} i^{-+}+i^{--}$. Since the restriction to $H_{1}\left(K_{i j}\right)$ of the homomorphism $\alpha$ is equal to $\left(t_{i} t_{j} i^{++}-t_{i} i^{+-}-t_{j} i^{-+}+\right.$ $\left.i^{--}\right) \prod_{n \neq i, j}\left(t_{n}-1\right)$, we get the second set of relations stated in the theorem.

Computation of $\varphi_{K}$. Finally, let us deal (a little faster) with the inclusion homomorphism

$$
\varphi_{K}: H_{1}\left(K_{\mu}^{ \pm}\right) \otimes \Lambda \rightarrow H_{1}\left(\widetilde{\Gamma}_{\mu}\right) .
$$

By an Euler characteristic argument, the rank of $H_{1}\left(K_{\mu}^{ \pm}\right)$is equal to $4\left(\begin{array}{c}\mu \\ 2\end{array}\right)-2 \mu+1$. Moreover, a recursive use of the Mayer-Vietoris exact sequence leads to the following fact: it is possible to present the $\Lambda$-module $H_{1}\left(\widetilde{\Gamma}_{\mu}\right)$ with $4\left(\begin{array}{c}\mu \\ 2\end{array}\right)-2 \mu+1$ generators and $\left(\begin{array}{l}\mu \\ 3\end{array}\right)$ relations. (The $\left(\begin{array}{l}\mu \\ 3\end{array}\right)$ relations correspond to all the possible 3 -dimensional 'cubes' in $\mathbb{Z}^{\mu}$.) One then checks that $\varphi_{K}$ is onto. Therefore, each relation in $H_{1}\left(\widetilde{\Gamma}_{\mu}\right)$ yields an element in $\operatorname{ker}\left(\varphi_{K}\right)$. One shows that the relation coming from the cube with coordinates $1 \leq i<j<k \leq \mu$ corresponds to the element

$t_{i} t_{j} t_{k} \gamma_{i j k}^{+++}-t_{i} t_{j} \gamma_{i j k}^{++-}-t_{i} t_{k} \gamma_{i j k}^{+-+}-t_{j} t_{k} \gamma_{i j k}^{-++}+t_{i} \gamma_{i j k}^{+--}+t_{j} \gamma_{i j k}^{-+-}+t_{k} \gamma_{i j k}^{--+}-\gamma_{i j k}^{---}$ in $\operatorname{ker}\left(\varphi_{K}\right)$, where $\gamma_{i j k}^{\varepsilon_{i} \varepsilon_{j} \varepsilon_{k}}$ is the 1-cycle in $H_{1}\left(K_{\mu}^{ \pm}\right)$joining $v_{i}^{\varepsilon_{i}}, v_{j}^{\varepsilon_{j}}$ and $v_{k}^{\varepsilon_{k}}$ in this order. The image under $\psi$ of this element is equal to $\prod_{n \neq i, j, k}\left(t_{n}-1\right)^{-1} \alpha\left(\gamma_{i j k}\right)$, completing the proof of the theorem.

\section{Piecewise continuity of the signature And nullity}

Let $L$ be a $\mu$-colored link. The signature and nullity of $L$ can be understood as functions

$$
\sigma_{L}, \eta_{L}: T_{*}^{\mu}: \longrightarrow \mathbb{Z}
$$

where $T_{*}^{\mu}=\left(S^{1} \backslash\{1\}\right)^{\mu} \subset \mathbb{C}^{\mu}$. In this subsection, we prove the following 'piecewise continuity' result.

Theorem 4.1. Let $E_{r}(L) \subset \Lambda_{\mu}$ be the $r^{\text {th }}$ Alexander ideal of $L$, and set

$$
\Sigma_{r}=\left\{\omega \in T_{*}^{\mu} \mid p(\omega)=0 \text { for all } p \in E_{r-1}(L)\right\} .
$$

This yields a finite sequence $T_{*}^{\mu}=\Sigma_{0} \supset \Sigma_{1} \supset \cdots \supset \Sigma_{\ell-1} \supset \Sigma_{\ell}=\emptyset$ such that, for all $r, \eta_{L}$ is equal to $r$ on $\Sigma_{r} \backslash \Sigma_{r+1}$, and $\sigma_{L}$ is constant on the connected components of $\Sigma_{r} \backslash \Sigma_{r+1}$.

Proof. Let $S=S_{1} \cup \cdots \cup S_{\mu}$ be a C-complex for $L$. Since $\sigma_{L}$ and $\eta_{L}$ remain unchanged by transformations $(T 1)$ and $(T 2)$ (recall Lemma 2.2 and the proof of Theorem 2.1), it may be assumed that $S_{i}$ is connected for all $i$, and $S_{i} \cap S_{j} \neq \emptyset$ for all $i \neq j$. In this case, Corollary 3.6 implies that

$$
E_{r}(L) \otimes_{\Lambda_{\mu}} \Lambda_{\mu}^{\prime}=E_{r}(A(t)) \otimes_{\Lambda_{\mu}} \Lambda_{\mu}^{\prime},
$$

where $A(t)=A\left(t_{1}, \ldots, t_{\mu}\right)=\sum_{\varepsilon} \varepsilon_{1} \cdots \varepsilon_{\mu} t_{1}^{\frac{1-\varepsilon_{1}}{2}} \cdots t_{\mu}^{\frac{1-\varepsilon_{\mu}}{2}} A^{\varepsilon}$. Hence, a given $\omega \in T_{*}^{\mu}$ belongs to $\Sigma_{r}$ if and only if $p(\omega)=0$ for all $p \in E_{r-1}(A(t))$. Now, recall that $\sigma_{L}(\omega)$ and $\eta_{L}(\omega)$ are defined as the signature and nullity of the Hermitian matrix $H(\omega)=\prod_{i=1}^{\mu}\left(1-\bar{\omega}_{i}\right) A(\omega)$. By this equality, $p(\omega)=0$ for all $p \in E_{r-1}(A(t))$ if 
and only if all the $(n-r+1) \times(n-r+1)$ minors of $H(\omega)$ vanish, where $n$ is the dimension of $H(\omega)$. This occurs if and only if the nullity of $H(\omega)$ is greater than or equal to $r$. Therefore, $\omega \in T_{*}^{\mu}$ belongs to $\Sigma_{r}$ if and only if $\eta_{L}(\omega) \geq r$. This gives the first part of the theorem. The second part is a consequence of the first point and of the following claim.

Claim: Let $x \mapsto H(x)$ be a continuous path in the space of $n$-dimensional Hermitian matrices. If the nullity of $H(x)$ is constant along the path, then the signature of $H(x)$ is constant as well.

Indeed, consider the continuous path $p:[0,1] \rightarrow \mathbb{C}[\lambda]$, where $p(x)$ is the characteristic polynomial of $H(x)$. Since $H(x)$ is Hermitian, the roots of $p(x)$ are real. Furthermore, they depend continuously on the coefficients of $p(x)$. In other words, the eigenvalues of $H(x)$ are real continuous functions of $x$. The nullity of $H(x)$ counts the number of these eigenvalues which are zero. Hence, if this number is constant, the sign of the eigenvalues cannot change. Therefore, the signature of $H(x)$ is constant.

As a corollary, we obtain the following result that extends a well known property of the Levine-Tristram signature. Note that in the case $l k\left(L_{i}, L_{j}\right)=0$, it is due to the second author (see Section 6] and [9, theorems 3.7 and 3.8]).

Corollary 4.2. Let $L$ be a $\mu$-colored link, and let $C$ denote the complement in $T_{*}^{\mu}$ of the zeroes of its Alexander polynomial. Then, $\sigma_{L}$ is constant on the connected components of $C$ and $\eta_{L}$ vanishes on $C$.

Proof. By Theorem 4.1, we just need to check that $C=T_{*}^{\mu} \backslash \Sigma_{1}$, that is: if $\omega \in T_{*}^{\mu}$, then $p(\omega)=0$ for all $p \in E_{0}(L)$ if and only if $\Delta_{L}(\omega)=0$. Corollary 3.6 implies that $E_{0}(L) \otimes_{\Lambda_{\mu}} \Lambda_{\mu}^{\prime}=E_{0}(A(t)) \otimes_{\Lambda_{\mu}} \Lambda_{\mu}^{\prime}$. Since $A(t)$ is a square matrix, $E_{0}(A(t))$ is the principal ideal generated by $\operatorname{det}(A) \doteq \prod_{i}\left(1-t_{i}\right)^{m_{i}} \cdot \Delta_{L}$. This proves the corollary.

Before concluding this section, let us look back at the 2-colored link $L$ given in Example 2.4. By Corollary 3.4, a presentation matrix of its Alexander module is given by $\left(t_{1} t_{2}+1\right)$. Therefore, $E_{0}(L)=\left(t_{1} t_{2}+1\right)$ and $E_{r}(L)=\Lambda_{2}$ for $r \geq 1$, leading to

$$
\Sigma_{r}= \begin{cases}T_{*}^{2} & \text { if } r \leq 0 \\ \left\{\left(\omega_{1}, \omega_{2}\right) \in T_{*}^{2} \mid \omega_{1} \omega_{2}+1=0\right\} & \text { if } r=1 \\ \emptyset & \text { if } r \geq 2\end{cases}
$$

By Theorem 4.1, $\sigma_{L}$ is constant on the connected components of $T_{*}^{2} \backslash \Sigma_{1}$ and of $\Sigma_{1}$. Furthermore, $\eta_{L}$ is equal to 0 on $T_{*}^{2} \backslash \Sigma_{1}$ and equal to 1 on $\Sigma_{1}$. This coincides with the computations made in Example 2.4.

\section{ON THE COMPUTATION BY LOCAL MOVES}

We now present and generalize an idea of J. Conway (see [6, §7.10] and [26. Lemma 3.1]) leading to a purely combinatorial computation of $\sigma_{L}$ for many colored links. Recall that for any $\mu$-colored link $L$, there exists a well-defined invariant $\nabla_{L}\left(t_{1}, \ldots, t_{\mu}\right) \in \mathbb{Z}\left(t_{1}, \ldots, t_{\mu}\right)$ called the Conway potential function of $L$, which satisfies:

$$
\nabla_{L}\left(t_{1}, \ldots, t_{\mu}\right) \doteq \begin{cases}\frac{1}{t_{1}-t_{1}^{-1}} \Delta_{L}\left(t_{1}^{2}\right) & \text { if } \mu=1 \\ \Delta_{L}\left(t_{1}^{2}, \ldots, t_{\mu}^{2}\right) & \text { if } \mu>1\end{cases}
$$


This normalization of the Alexander polynomial was first introduced by J. Conway [5], and formally defined by R. Hartley [17]. Note that it is possible to compute this invariant from a colored link diagram using only combinatorial methods (see [23]).

Given a complex number $z=e^{i \theta}$ with $0<\theta<2 \pi$, we shall denote by $z^{1 / 2}$ the complex number $e^{i \theta / 2}$. If $\omega=\left(\omega_{1}, \ldots, \omega_{\mu}\right) \in T_{*}^{\mu}$, then $\omega^{1 / 2}=\left(\omega_{1}^{1 / 2}, \ldots, \omega_{\mu}^{1 / 2}\right)$. Also, we shall denote by $\operatorname{sgn}(\lambda)$ the sign of the real number $\lambda$.

Theorem 5.1. a) Let $L$ and $L^{\prime}$ be two colored links given by diagrams related by a single change as illustrated in Figure 16. For any $\omega \in T_{*}^{\mu}$ such that $\nabla_{L^{\prime}}\left(\omega^{1 / 2}\right) \neq 0$, $i \frac{\nabla_{L}\left(\omega^{1 / 2}\right)}{\nabla_{L^{\prime}}\left(\omega^{1 / 2}\right)}$ is a real number and

$$
\sigma_{L}(\omega)=\sigma_{L^{\prime}}(\omega)+\operatorname{sgn}\left(i \frac{\nabla_{L}\left(\omega^{1 / 2}\right)}{\nabla_{L^{\prime}}\left(\omega^{1 / 2}\right)}\right) .
$$

Conversely, for any $\omega \in T_{*}^{\mu}$ such that $\nabla_{L}\left(\omega^{1 / 2}\right) \neq 0$,

$$
\sigma_{L^{\prime}}(\omega)=\sigma_{L}(\omega)+\operatorname{sgn}\left(i \frac{\nabla_{L^{\prime}}\left(\omega^{1 / 2}\right)}{\nabla_{L}\left(\omega^{1 / 2}\right)}\right) .
$$

b) Consider colored links $L$ and $L^{\prime \prime}$ which differ by one of the local moves described in Figure 17. If $\omega \in T_{*}^{\mu}$ satisfies $\nabla_{L^{\prime \prime}}\left(\omega^{1 / 2}\right) \neq 0$, then $\frac{\nabla_{L}\left(\omega^{1 / 2}\right)}{\nabla_{L^{\prime \prime}}\left(\omega^{1 / 2}\right)}$ is a real number and

$$
\sigma_{L}(\omega)=\sigma_{L^{\prime \prime}}(\omega)+\delta \cdot \operatorname{sgn}\left(\frac{\nabla_{L}\left(\omega^{1 / 2}\right)}{\nabla_{L^{\prime \prime}}\left(\omega^{1 / 2}\right)}\right),
$$

with $\delta= \pm 1$ as in Figure 17. Conversely, if $\omega \in T_{*}^{\mu}$ satisfies $\nabla_{L}\left(\omega^{1 / 2}\right) \neq 0$, then

$$
\sigma_{L^{\prime \prime}}(\omega)=\sigma_{L}(\omega)-\delta \cdot \operatorname{sgn}\left(\frac{\nabla_{L^{\prime \prime}}\left(\omega^{1 / 2}\right)}{\nabla_{L}\left(\omega^{1 / 2}\right)}\right) .
$$

Given $L$ a link, let $\Delta_{L}(t)$ denote its Alexander-Conway polynomial $\Delta_{L}(t)=$ $\left(t^{1 / 2}-t^{-1 / 2}\right) \nabla_{L}\left(t^{1 / 2}\right)$. The theorem above implies the following result for the Levine-Tristram signature.

Corollary 5.2. Let $L$ and $L^{\prime}$ be two links related by a single change as illustrated in Figure 16. For any $\omega \in S^{1} \backslash\{1\}$ such that $\Delta_{L^{\prime}}(\omega) \neq 0, i \frac{\Delta_{L^{\prime}}(\omega)}{\Delta_{L}(\omega)} \in \mathbb{R}$ and

$$
\sigma_{L}(\omega)=\sigma_{L^{\prime}}(\omega)+\operatorname{sgn}\left(i \frac{\Delta_{L}(\omega)}{\Delta_{L^{\prime}}(\omega)}\right) .
$$

Furthermore, for any $\omega \in T_{*}^{\mu}$ such that $\Delta_{L}(\omega) \neq 0$,

$$
\sigma_{L^{\prime}}(\omega)=\sigma_{L}(\omega)+\operatorname{sgn}\left(i \frac{\Delta_{L^{\prime}}(\omega)}{\Delta_{L}(\omega)}\right) .
$$

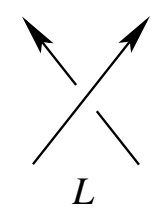
Figure 16. The local move in part a) of T
strands are assumed to have the same color. 

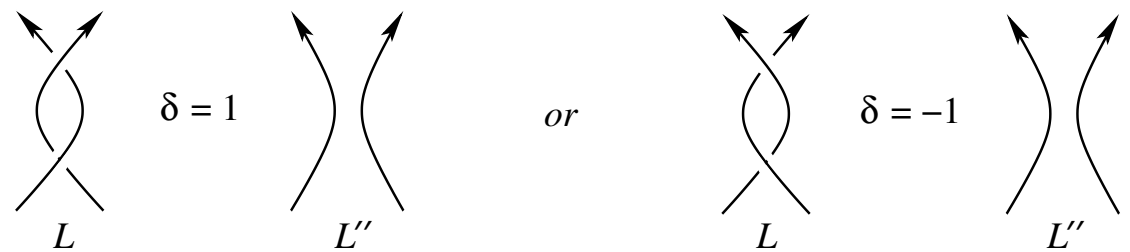

Figure 17. The local moves in part b) of Theorem 5.1. The colors of the two strands are assumed to be distinct.

Let us postpone the proof of Theorem 5.1 to the end of the section. We shall first discuss to what extent this result leads to an algorithm for the computation of the signature of a colored link. Let us start with the case of a knot $K$. Corollary 5.2 provides the following combinatorial algorithm for the computation of $\sigma_{K}(\omega)$ for all but a finite number of $\omega$ in $S^{1}$. Consider a diagram for $K$ with $n$ double points and no 'nugatory crossings' (that is, a diagram that remains connected after the move of Figure 16]. Apply the local move of Figure 16 to any crossing. We get a 2-component link $L^{\prime}$ given by a connected diagram. Apply the same local move to any crossing between the two components of $L^{\prime}$. (Such a crossing exists since the diagram is connected.) This yields a knot $K^{\prime \prime}$ given by a diagram with $n-2$ crossings. Corollary 5.2 gives the equality

$$
\sigma_{K}(\omega)=\sigma_{K^{\prime \prime}}(\omega)-\operatorname{sgn}\left(i \frac{\Delta_{L^{\prime}}(\omega)}{\Delta_{K}(\omega)}\right)+\operatorname{sgn}\left(i \frac{\Delta_{L^{\prime}}(\omega)}{\Delta_{K^{\prime \prime}}(\omega)}\right)
$$

for all $\omega$ such that $\Delta_{K}(\omega) \Delta_{K^{\prime \prime}}(\omega) \neq 0$. Since both $K$ and $K^{\prime \prime}$ are knots, $\Delta_{K} \Delta_{K^{\prime \prime}}$ is non-zero. Hence, the relation above holds for all but a finite number of $\omega$ in $S^{1}$. We are done by induction on $n$.

Example 5.3. Let $K$ be the right-hand trefoil knot. Consider the transformations of $K$ illustrated in Figure 18. One easily computes $\Delta_{K}(t)=t-1+t^{-1}$. Moreover, $L^{\prime}$ is the positive Hopf link and $K^{\prime \prime}$ the trivial knot, so $\Delta_{L^{\prime}}(t)=t^{1 / 2}-t^{-1 / 2}$ and $\Delta_{K^{\prime \prime}}(t)=1$. This gives

$$
\begin{aligned}
\sigma_{K}(\omega) & =\sigma_{K^{\prime \prime}}(\omega)-\operatorname{sgn}\left(i \frac{\omega^{1 / 2}-\overline{\omega^{1 / 2}}}{\omega-1+\bar{\omega}}\right)+\operatorname{sgn}\left(i\left(\omega^{1 / 2}-\overline{\omega^{1 / 2}}\right)\right) \\
& =\operatorname{sgn}\left(\frac{1}{\omega-1+\bar{\omega}}\right)-1 .
\end{aligned}
$$

On this particularly simple example, $\Delta_{L^{\prime}}$ never vanishes on $S^{1} \backslash\{1\}$. Hence, Corollary 5.2 in fact leads to the formula

$$
\sigma_{K}(\omega)=\operatorname{sgn}(\omega-1+\bar{\omega})-1
$$

which holds for any value of $\omega$.

Now, let $L=K_{1} \cup \cdots \cup K_{\mu}$ be a $\mu$-component $\mu$-colored link. Theorem 5.1 b) leads to the following result: there exists a $\mu$-variable polynomial $\Delta$ such that, for all $\omega=\left(\omega_{1}, \ldots, \omega_{\mu}\right)$ in $T^{\mu}$ with $\Delta(\omega) \neq 0$, the computation of $\sigma_{L}(\omega)$ boils down to the computation of $\sigma_{K_{i}}\left(\omega_{i}\right)$ for $i=1, \ldots, \mu$. And we just saw that for all $i, \sigma_{K_{i}}\left(\omega_{i}\right)$ can be computed for all but a finite number of $\omega_{i}$ in $S^{1}$. However, for some links $L$, the polynomial $\Delta$ is always zero and the result above is useless. For example, 


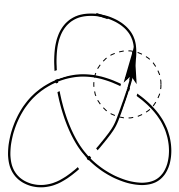

K

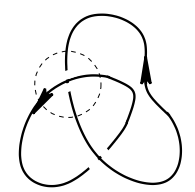

$L^{\prime}$

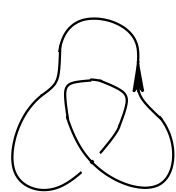

$K^{\prime \prime}$

FiguRE 18.

consider an irreducible boundary link $L$ with $\mu \geq 3$ components. Let $L^{\prime}$ be any $\mu$ colored link obtained from $L$ by one of the local moves described in Figures 16 and 17. Then, it is easy to check that both $\nabla_{L}$ and $\nabla_{L^{\prime}}$ are identically zero. Therefore, Theorem 5.1 is of no use for the computation of $\sigma_{L}$ in this particular case.

However, the method described above does lead to an algorithm for links that are not 'algebraically split'. Recall that a $\mu$-component link $L$ is said to be algebraically split if there is an ordering of its components $K_{1}, \ldots, K_{\mu}$ and an integer $1 \leq k<\mu$ such that $l k\left(K_{i}, K_{j}\right)=0$ for all $1 \leq i \leq k<j \leq \mu$. Using the Torres formula, one checks that if $L$ is not algebraically split, then $\nabla_{L}$ is non-zero. Using this result, one easily sees that if $L$ is not algebraically split, then the polynomial $\Delta$ described above can be chosen to be non-zero. Therefore, $\sigma_{L}(\omega)$ can be computed for all $\omega$ in the complement in $T^{\mu}$ of the zeroes of some non-zero polynomial. Let us illustrate this with an example.

Example 5.4. Consider the colored link $L$ given in Example 2.4. Also, let $L^{\prime \prime}$ be the 2 -colored positive Hopf link. Since $\nabla_{L}\left(t_{1}, t_{2}\right)=t_{1} t_{2}+t_{1}^{-1} t_{2}^{-1}$ and $\nabla_{L^{\prime \prime}}\left(t_{1}, t_{2}\right)=1$, Theorem $5.1 \mathrm{~b}$ ) gives

$$
\sigma_{L}\left(\omega_{1}, \omega_{2}\right)=\sigma_{L^{\prime \prime}}\left(\omega_{1}, \omega_{2}\right)+\operatorname{sgn}\left(\omega_{1}^{1 / 2} \omega_{2}^{1 / 2}+\overline{\omega_{1}^{1 / 2} \omega_{2}^{1 / 2}}\right) .
$$

By Theorem 5.1 b) again (or since there is a contractible C-complex for $L^{\prime \prime}$ ), the signature of $L^{\prime \prime}$ is zero. Setting $\omega_{1}=e^{i \theta_{1}}$ and $\omega_{2}=e^{i \theta_{2}}$, we get

$$
\sigma_{L}\left(\theta_{1}, \theta_{2}\right)=\operatorname{sgn}\left(\cos \left(\left(\theta_{1}+\theta_{2}\right) / 2\right)\right) .
$$

This coincides with the result given in Example 2.4

The proof of Theorem 5.1 rests upon several lemmas.

Lemma 5.5. Let $H$ be a d-dimensional Hermitian matrix. Then its signature $\sigma$ and nullity $\eta$ satisfy $\sigma+\eta \equiv d(\bmod 2)$. Furthermore, if $\eta=0$, then $\sigma \equiv d(\bmod 4)$ if and only if $\operatorname{det}(H)>0$.

Proof. Let $m$ be the number of negative eigenvalues of $H$. We have

$$
\sigma+\eta=(d-m-\eta)-m+\eta=d-2 m \equiv d \quad(\bmod 2) .
$$

If $\eta=0$, then $\operatorname{det}(H)>0$ if and only if $m$ is even, that is, if and only if $\sigma=$ $d-2 m \equiv d(\bmod 4)$.

Lemma 5.6. Let $L$ be a colored link with $\nu$ connected components. Then, for all $\omega \in T_{*}^{\mu}$,

$$
\sigma_{L}(\omega)+\eta_{L}(\omega) \equiv \nu+\sum_{i<j} l k\left(L_{i}, L_{j}\right)+1 \quad(\bmod 2)
$$


Proof. Let $S$ be a connected C-complex for $L$. Applying Lemma 5.5 to the matrix $H(\omega)$, we have $\sigma_{L}(\omega)+\eta_{L}(\omega) \equiv \operatorname{rk} H_{1}(S)(\bmod 2)$. An elementary Euler characteristic argument shows that $\operatorname{rk} H_{1}(S) \equiv \nu+\#\{$ clasps of $S\}+1(\bmod 2)$. Finally, the number of clasps of $S$ clearly has the same parity as $\sum_{i<j} l k\left(L_{i}, L_{j}\right)$.

Lemma 5.7. Let $L$ be a colored link with $\nu$ connected components. Then, for any $\omega \in T_{*}^{\mu}$ such that $\eta_{L}(\omega)=0$, we have

$$
\sigma_{L}(\omega) \equiv \nu+\sum_{i<j} l k\left(L_{i}, L_{j}\right)-\operatorname{sgn}\left(i^{\nu} \nabla_{L}\left(\omega^{1 / 2}\right)\right) \quad(\bmod 4) .
$$

Proof. Let $S$ be a connected C-complex for $L$. Fix $\omega \in T_{*}^{\mu}$ such that $\eta_{L}(\omega)=0$. If $z_{j}$ denotes the complex number $\omega_{j}^{1 / 2}$, we have

$$
\begin{aligned}
\overline{H(\omega)} & =\prod_{j=1}^{\mu}\left(1-\omega_{j}\right) \sum_{\varepsilon} \varepsilon_{1} \cdots \varepsilon_{\mu} \omega_{1}^{\frac{\varepsilon_{1}-1}{2}} \cdots \omega_{\mu}^{\frac{\varepsilon_{\mu}-1}{2}} A^{\varepsilon} \\
& =\prod_{j=1}^{\mu}\left(\bar{z}_{j}^{2}-1\right) \sum_{\varepsilon} \varepsilon_{1} \cdots \varepsilon_{\mu} z_{1}^{\varepsilon_{1}+1} \cdots z_{\mu}^{\varepsilon_{\mu}+1} A^{\varepsilon} \\
& =\prod_{j=1}^{\mu}\left(\bar{z}_{j}-z_{j}\right) B\left(z_{1}, \ldots, z_{\mu}\right),
\end{aligned}
$$

where $B\left(z_{1}, \ldots, z_{\mu}\right)$ denotes the matrix $\sum_{\varepsilon} \varepsilon_{1} \cdots \varepsilon_{\mu} z_{1}^{\varepsilon_{1}} \cdots z_{\mu}^{\varepsilon_{\mu}} A^{\varepsilon}$. On the other hand, we know by [4] that the Conway potential function of $L$ is given by

$$
\nabla_{L}\left(t_{1}, \ldots, t_{\mu}\right)=(-1)^{\frac{c-\ell}{2}} \prod_{j=1}^{\mu}\left(t_{j}-t_{j}^{-1}\right)^{\chi\left(S \backslash S_{j}\right)-1} \operatorname{det}\left(-B\left(t_{1}, \ldots, t_{\mu}\right)\right),
$$

where $c$ is the number of clasps of $S$ and $\ell=\sum_{i<j} l k\left(L_{i}, L_{j}\right)$. Let $\beta_{1}$ denote the first Betti number of $S$. By Lemma [5.5. $\sigma_{L}(\omega) \equiv \beta_{1}(\bmod 4)$ if and only if

$$
\begin{aligned}
0 & <\operatorname{det}(H(\omega))=\operatorname{det}\left(\prod_{j=1}^{\mu}\left(\bar{z}_{j}-z_{j}\right) B\left(z_{1}, \ldots, z_{\mu}\right)\right) \\
& =(-1)^{\frac{c-\ell}{2}+\beta_{1}+\mu \beta_{1}} \prod_{j=1}^{\mu}\left(z_{j}-\bar{z}_{j}\right)^{1-\chi\left(S \backslash S_{j}\right)+\beta_{1}} \nabla_{L}\left(z_{1}, \ldots, z_{\mu}\right) .
\end{aligned}
$$

Since $z_{j}=e^{i \theta}$ with $0<\theta<\pi, z_{j}-\bar{z}_{j}=\lambda i$ with $\lambda \in \mathbb{R}_{+}^{*}$. Therefore, $\sigma_{L}(\omega) \equiv \beta_{1}$ $(\bmod 4)$ if and only if $0<i^{\alpha} \nabla_{L}\left(z_{1}, \ldots, z_{\mu}\right)$, with

$$
\alpha=c-\ell+2 \beta_{1}-2 \mu \beta_{1}+\sum_{j=1}^{\mu}\left(1-\chi\left(S \backslash S_{j}\right)+\beta_{1}\right) .
$$

An Euler characteristic argument shows that $\alpha=1-\ell+\beta_{1}$. We know from Lemma 5.6 that $\beta_{1} \equiv \nu+\ell+1(\bmod 2)$. Via the transformation $(T 2)$ of Figure 3 , it may be assumed that $\beta_{1} \equiv \nu+\ell-1(\bmod 4)$. Therefore, $\sigma_{L}(\omega) \equiv \nu+\ell-1(\bmod 4)$ if and only if $i^{\nu} \nabla_{L}\left(z_{1}, \ldots, z_{\mu}\right)>0$. This fact, together with Lemma 5.6, gives the result.

The following lemma is a direct consequence of Theorem 4.1. Nevertheless, we now give an alternative proof. 
Lemma 5.8. Given any colored link $L$ and any $\omega \in T_{*}^{\mu}, \eta_{L}(\omega)=0$ if and only if $\nabla_{L}\left(\omega^{1 / 2}\right) \neq 0$.

Proof. We saw in the proof of Lemma 5.7 that

$$
\operatorname{det}(H(\omega))= \pm \prod_{j}\left(\omega_{j}^{1 / 2}-\overline{\omega_{j}^{1 / 2}}\right)^{m_{j}} \nabla_{L}\left(\omega^{1 / 2}\right)
$$

for some integers $m_{j}$. Note that $\eta_{L}(\omega) \neq 0$ if and only if $\operatorname{det}(H(\omega))=0$. Since $\omega_{j}^{1 / 2} \neq \overline{\omega_{j}^{1 / 2}}$, the lemma is checked.

Proof of Theorem 5.1, a) Let $S^{\prime}$ be a connected C-complex for $L^{\prime}$. A C-complex $S$ for $L$ is obtained from $S^{\prime}$ by attaching a band with a half-twist. Fix $\omega \in T_{*}^{\mu}$. If $H^{\prime}(\omega)$ and $H(\omega)$ denote the associated Hermitian matrices, then clearly

$$
H(\omega)=\left(\begin{array}{cc}
H^{\prime}(\omega) & v \\
\bar{v}^{T} & \lambda
\end{array}\right)
$$

for some complex vector $v$ and real number $\lambda$. Therefore,

$$
\left|\eta_{L^{\prime}}(\omega)-\eta_{L}(\omega)\right|+\left|\sigma_{L^{\prime}}(\omega)-\sigma_{L}(\omega)\right|=1 .
$$

Let us now assume that $\nabla_{L^{\prime}}\left(\omega^{1 / 2}\right) \neq 0$. By Lemma [5.8, $\eta_{L^{\prime}}(\omega)=0$. If $\nabla_{L}\left(\omega^{1 / 2}\right)=$ 0 , then $\eta_{L}(\omega)>0$. By equation $(\star), \sigma_{L^{\prime}}(\omega)=\sigma_{L}(\omega)$, so the theorem holds in this case. Let us now assume that $\nabla_{L}\left(\omega^{1 / 2}\right) \neq 0$. By Lemma 5.8 and equation $(\star)$, we have $\sigma_{L}(\omega)=\sigma_{L^{\prime}}(\omega)+\varepsilon$ for some $\varepsilon= \pm 1$. Reducing this equation modulo 4 , Lemma 5.7 implies

$$
\nu+\ell-\operatorname{sgn}\left(i^{\nu} \nabla_{L}\left(\omega^{1 / 2}\right)\right) \equiv \nu^{\prime}+\ell^{\prime}-\operatorname{sgn}\left(i^{\nu^{\prime}} \nabla_{L^{\prime}}\left(\omega^{1 / 2}\right)\right)+\varepsilon \quad(\bmod 4),
$$

where $\ell=\sum_{i<j} l k\left(L_{i}, L_{j}\right)$ and $\ell^{\prime}=\sum_{i<j} l k\left(L_{i}^{\prime}, L_{j}^{\prime}\right)$. Hence,

$$
\varepsilon \equiv\left(\nu-\nu^{\prime}\right)+\left(\ell-\ell^{\prime}\right)+\operatorname{sgn}\left(i^{\nu^{\prime}} \nabla_{L^{\prime}}\left(\omega^{1 / 2}\right)\right)-\operatorname{sgn}\left(i^{\nu} \nabla_{L}\left(\omega^{1 / 2}\right)\right) \quad(\bmod 4) .
$$

Clearly, $\ell=\ell^{\prime}$ and $\nu=\nu^{\prime}+\tau$ with $\tau= \pm 1$. Therefore,

$$
\varepsilon \equiv \tau+\operatorname{sgn}\left(i^{\nu^{\prime}} \nabla_{L^{\prime}}\left(\omega^{1 / 2}\right)\right)-\operatorname{sgn}\left(i^{\nu} \nabla_{L}\left(\omega^{1 / 2}\right)\right) \quad(\bmod 4) .
$$

Since $\varepsilon= \pm 1$ and $\tau= \pm 1$, this implies

$$
\varepsilon=\operatorname{sgn}\left(i^{1-\tau} \frac{i^{\nu} \nabla_{L}\left(\omega^{1 / 2}\right)}{i^{\nu^{\prime}} \nabla_{L^{\prime}}\left(\omega^{1 / 2}\right)}\right)=\operatorname{sgn}\left(i \frac{\nabla_{L}\left(\omega^{1 / 2}\right)}{\nabla_{L^{\prime}}\left(\omega^{1 / 2}\right)}\right),
$$

so the first equality is checked. The second equality can be derived from this one using Figure 19.
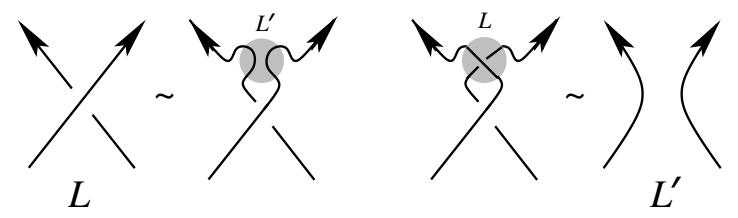

Figure 19.

b) Given $S^{\prime \prime}$ a connected C-complex for $L^{\prime \prime}$, a C-complex $S$ for $L$ is obtained from $S^{\prime \prime}$ by attaching a clasp. As in the proof of $a$ ), this leads to the equation $\sigma_{L}(\omega)=\sigma_{L^{\prime \prime}}(\omega)+\varepsilon$ for some $\varepsilon= \pm 1$ satisfying

$$
\varepsilon \equiv\left(\nu-\nu^{\prime \prime}\right)+\left(\ell-\ell^{\prime \prime}\right)+\operatorname{sgn}\left(i^{\nu^{\prime \prime}} \nabla_{L^{\prime \prime}}\left(\omega^{1 / 2}\right)\right)-\operatorname{sgn}\left(i^{\nu} \nabla_{L}\left(\omega^{1 / 2}\right)\right) \quad(\bmod 4) .
$$


This time, $\nu=\nu^{\prime \prime}$ and $\ell=\ell^{\prime \prime}+\delta$, with $\delta= \pm 1$ as described in the statement of the theorem. Hence,

$$
\varepsilon \equiv \delta+\operatorname{sgn}\left(i^{\nu} \nabla_{L^{\prime \prime}}\left(\omega^{1 / 2}\right)\right)-\operatorname{sgn}\left(i^{\nu} \nabla_{L}\left(\omega^{1 / 2}\right)\right) \quad(\bmod 4) .
$$

Since $\varepsilon= \pm 1$ and $\delta= \pm 1$, this implies

$$
\varepsilon=\delta \cdot \operatorname{sgn}\left(\frac{i^{\nu} \nabla_{L}\left(\omega^{1 / 2}\right)}{i^{\nu} \nabla_{L^{\prime \prime}}\left(\omega^{1 / 2}\right)}\right)=\delta \cdot \operatorname{sgn}\left(\frac{\nabla_{L}\left(\omega^{1 / 2}\right)}{\nabla_{L^{\prime \prime}}\left(\omega^{1 / 2}\right)}\right) .
$$

The last equality is a consequence of this equation.

\section{The 4-Dimensional VIEWPoint}

In this section, we present the signatures $\sigma_{L}(\omega)$ from the point of view of coverings and intersection forms. Roughly speaking, we show that they can be constructed as the Atiyah-Singer invariant of a finite abelian covering of the link exterior in $S^{3}$. In doing this, we also relate our signatures to invariants introduced by Gilmer [12, Smolinski [31, Levine 21] and the second author [9].

Definition. Let $F$ be a union of compact connected oriented smooth surfaces $F_{1}, \ldots, F_{\mu}$ in $B^{4}$. We shall say that $F$ has boundary $L$ if the following holds:

(i) For all $i, F_{i}$ is smoothly embedded in $B^{4}$ and $\partial F_{i}=L_{i}$.

(ii) For all $i \neq j, F_{i}$ and $F_{j}$ intersect transversally in a finite number of points, possibly empty.

(iii) For all $i, j, k$ pairwise distinct, $F_{i} \cap F_{j} \cap F_{k}$ is empty.

The existence of a surface $F$ with boundary $L$ is obvious. For such an $F$, denote by $W_{F}$ (or simply by $W$ ) the complement of an open tubular neighborhood of $F$ in $B^{4}$. By the exact sequence of the pair $\left(B^{4}, B^{4} \backslash F\right)$ and duality, one shows that $H_{1}\left(W_{F}\right)$ is the free abelian group generated by the meridians $m_{1}, \ldots, m_{\mu}$ of the components $F_{1}, \ldots, F_{\mu}$ of $F$. Therefore, the group of characters $\operatorname{Hom}\left(H_{1}\left(W_{F}\right), S^{1}\right)$ can be identified with the $\mu$ dimensional torus $T^{\mu}$. To the element $\omega=\left(\omega_{1}, \ldots, \omega_{\mu}\right)$ of $T^{\mu}$ corresponds the character $\chi_{\omega}$ given by $\chi_{\omega}\left(m_{i}\right)=\omega_{i}$.

Let $T_{\mathbb{Q}}^{\mu}$ be the subset of $T_{*}^{\mu}$ constituted by the points with rational coordinates, i.e.

$$
T_{\mathbb{Q}}^{\mu}=\left\{\left(\omega_{1}, \ldots, \omega_{\mu}\right) \in T^{\mu} ; \omega_{j}=e^{2 i \pi \theta_{j}} \text { with } \theta_{j} \in \mathbb{Q} \cap\right] 0,1[\} .
$$

Consider an element $\omega$ of $T_{\mathbb{Q}}^{\mu}$. The image of the corresponding character $\chi_{\omega}$ is the subgroup of $\mathbb{C}^{*}$ generated by $\alpha=e^{2 i \pi / q}$, where $q$ is the least common multiple of the orders of the $\omega_{i}$ 's. Hence, $\chi_{\omega}$ induces a $q$-fold cyclic covering $W^{q} \rightarrow W$ with a canonical deck transformation $\tau$ generating $C_{q}$, the group of the covering. The cellular chain complex $C_{*}\left(W^{q}\right)$ is a $\mathbb{Z}\left[C_{q}\right]$-module, and so is $\mathbb{C}$ via the homomorphism $j: C_{q} \rightarrow \mathbb{C}^{*}$ which sends $\tau$ to $\alpha$. The twisted homology of $(W, \omega)$, denoted by $H_{*}^{\omega}(W ; \mathbb{C})$, is the homology of the chain complex $C_{*}\left(W^{q}\right) \otimes_{\mathbb{Z}\left[C_{q}\right]} \mathbb{C}$. Note that $\mathbb{C}$ is flat over $\mathbb{Z}\left[C_{q}\right]$, so

$$
H_{*}^{\omega}(W ; \mathbb{C})=H_{*}\left(W^{q}\right) \otimes_{\mathbb{Z}\left[C_{q}\right]} \mathbb{C} .
$$

The twisted homology $H_{*}^{\omega}(X ; \mathbb{C})$ of the exterior of $L$ in $S^{3}$ is defined similarly, using the character of $H_{1}(X)$ induced by $\chi_{\omega}$. 
Definition. For $\omega$ in $T_{\mathbb{Q}}^{\mu}$, let $\varphi_{F}^{\omega}$ (or simply $\varphi^{\omega}$ ) denote the twisted intersection form defined by

$$
\begin{aligned}
\varphi_{F}^{\omega}: H_{2}^{\omega}\left(W_{F} ; \mathbb{C}\right) \times H_{2}^{\omega}\left(W_{F} ; \mathbb{C}\right) & \longrightarrow \mathbb{C}, \\
(x \otimes u, y \otimes v) & \longmapsto u \bar{v} \sum_{i=1}^{q}\left\langle x, \tau^{i} y\right\rangle \alpha^{i}
\end{aligned}
$$

for $x, y \in H_{2}\left(W^{q}\right)$ and $u, v \in \mathbb{C}$. Here, $\langle$,$\rangle is the intersection form induced by the$ orientation of $W$ lifted to $W^{q}$, and $\lambda \mapsto \bar{\lambda}$ is the complex conjugation.

Note that $\varphi^{\omega}$ is a well-defined Hermitian form. In fact, it is conjugate to the ordinary intersection form on $H_{2}\left(W^{q} ; \mathbb{C}\right)$ restricted to the eigenspace of $\tau$ with eigenvalue $\alpha$ (see Lemma 6.2 below).

This section is mainly devoted to the proof of the following theorem.

Theorem 6.1. Let $L$ be a $\mu$-colored link. For any surface $F$ in $B^{4}$ with boundary $L$, and for all $\omega \in T_{\mathbb{Q}}^{\mu}$,

$$
\begin{aligned}
& \sigma_{L}(\omega)=\operatorname{sign}\left(\varphi_{F}^{\omega}\right), \\
& \eta_{L}(\omega)=\operatorname{dim} H_{1}^{\omega}(X ; \mathbb{C})=\operatorname{null}\left(\varphi_{F}^{\omega}\right)+\operatorname{dim} H_{1}^{\omega}\left(W_{F} ; \mathbb{C}\right)-\operatorname{dim} H_{3}^{\omega}\left(W_{F} ; \mathbb{C}\right) .
\end{aligned}
$$

6.1. Finite abelian coverings. In this subsection, we relate the twisted intersection forms of finite abelian coverings and of their cyclic quotients.

As above, let $W$ be the exterior of a surface $F$ with boundary $L$ (or more generally, any compact connected oriented 4-dimensional manifold with $H_{1}(W)=$ $\bigoplus_{i=1}^{\mu} \mathbb{Z}$, where the generator of the $i^{\text {th }}$ summand is denoted $\left.m_{i}\right)$. Consider integers $q_{1}, \ldots, q_{\mu}$ with $q_{i}>1$ for all $i$. The natural projection $\gamma: H_{1}(W) \rightarrow G=C_{q_{1}} \times$ $\cdots \times C_{q_{\mu}}$ mapping $m_{i}$ to a preferred generator $\tau_{i}$ of $C_{q_{i}}$ induces a finite abelian covering $W^{\gamma} \rightarrow W$. As usual, $H_{*}\left(W^{\gamma}\right)$ is a module over the group ring $\mathbb{Z}[G]$. Let $S: H_{2}\left(W^{\gamma}\right) \times H_{2}\left(W^{\gamma}\right) \rightarrow \mathbb{Z}[G]$ be the pairing given by

$$
S(x, y)=\sum_{g \in G}\langle x, g y\rangle g,
$$

where $\langle$,$\rangle is the intersection form induced by the orientation of W$ lifted to $W^{\gamma}$. Note that $S$ is sesquilinear with respect to the involution of $\mathbb{Z}[G]$ induced by $g \mapsto g^{-1}$.

Now, consider an element $\omega$ of $T_{\mathbb{Q}}^{\mu}$ with $\omega_{i}$ of order $q_{i}$ for all $i$. As above, let $q$ denote the least common multiple of the $q_{i}$ 's. The corresponding character $\chi_{\omega}: H_{1}(W) \rightarrow \mathbb{C}^{*}$ can be written $\chi_{\omega}=j \circ r_{\omega} \circ \gamma$, where $j: C_{q} \rightarrow \mathbb{C}^{*}$ satisfies $j(\tau)=\alpha$ and $r_{\omega}: G \rightarrow C_{q}$ is such that $j \circ r_{\omega}$ maps $\tau_{i}=\gamma\left(m_{i}\right)$ to $\omega_{i}$. Let $s_{\omega}: G \rightarrow \mathbb{C}^{*}$ denote the character given by $j \circ r_{\omega}$. It induces a homomorphism of rings $\mathbb{Z}[G] \rightarrow \mathbb{C}$ compatible with the involutions of these rings. In particular, it endows $\mathbb{C}$ with a structure of $\mathbb{Z}[G]$-module. Let $\psi^{\omega}$ (or $\psi_{F}^{\omega}$ ) denote the Hermitian form on $H_{2}\left(W^{\gamma}\right) \otimes_{\mathbb{Z}[G]} \mathbb{C}$ induced by the $\mathbb{Z}[G]$-sesquilinear form $S$ via the ring homomorphism $\mathbb{Z}[G] \rightarrow \mathbb{C}$. In other words, $\psi^{\omega}$ is given by

$$
\begin{aligned}
\psi^{\omega}: H_{2}\left(W^{\gamma}\right) \otimes_{\mathbb{Z}[G]} \mathbb{C} \times H_{2}\left(W^{\gamma}\right) \otimes_{\mathbb{Z}[G]} \mathbb{C} & \longrightarrow \mathbb{C}, \\
(x \otimes u, y \otimes v) & \longmapsto u \bar{v} \sum_{g \in G}\langle x, g y\rangle s_{\omega}(g) .
\end{aligned}
$$

Lemma 6.2. Given a character $s: G \rightarrow \mathbb{C}^{*}$, set

$$
E_{s}=\left\{x \in H_{2}\left(W^{\gamma} ; \mathbb{C}\right) ; g x=s(g) x \text { for all } g \in G\right\} \text {. }
$$

Then, $\psi^{\omega}$ is conjugate to the restriction to $E_{s_{\omega}}$ of the Hermitian intersection form on $H_{2}\left(W^{\gamma} ; \mathbb{C}\right)$. 
Proof. First, observe that $H_{2}\left(W^{\gamma}\right) \otimes_{\mathbb{Z}[G]} \mathbb{C}$ is equal to $H_{2}\left(W^{\gamma} ; \mathbb{C}\right) \otimes_{\mathbb{C}[G]} \mathbb{C}$. For all $s \in \mathcal{R}_{G}=\operatorname{Hom}\left(G, \mathbb{C}^{*}\right)$, let $\vartheta_{s}$ be the element of $\mathbb{C}[G]$ given by

$$
\vartheta_{s}=\frac{1}{|G|} \sum_{g \in G} \overline{s(g)} g \text {. }
$$

One easily checks that $g \vartheta_{s}=s(g) \vartheta_{s}$ for all $g \in G$ and $s \in \mathcal{R}_{G}$. This gives the inclusion $\vartheta_{s} H_{2}\left(W^{\gamma} ; \mathbb{C}\right) \subset E_{s}$. On the other hand, $\vartheta_{s} \vartheta_{s}=\vartheta_{s}$ and $\vartheta_{s} \vartheta_{s^{\prime}}=0$ if $s \neq s^{\prime}$. Hence, if $x$ belongs to $E_{s}$, then $\vartheta_{s^{\prime}} x=0$ for all $s \neq s^{\prime}$. Since $\sum_{s \in \mathcal{R}_{G}} \vartheta_{s}=1$ (see [29, Lemma 2.1]), we have $x=\vartheta_{s} x$ and the inclusion $E_{s} \subset \vartheta_{s} H_{2}\left(W^{\gamma} ; \mathbb{C}\right)$ is proved. In other words, there is a canonical isomorphism of $\mathbb{C}[G]$-modules

$$
H_{2}\left(W^{\gamma} ; \mathbb{C}\right) \simeq \bigoplus_{s \in \mathcal{R}_{G}} \vartheta_{s} H_{2}\left(W^{\gamma} ; \mathbb{C}\right)=\bigoplus_{s \in \mathcal{R}_{G}} E_{s}
$$

Since $G$ acts by isometries, this decomposition is orthogonal with respect to the intersection form $\langle$,$\rangle on H_{2}\left(W^{\gamma} ; \mathbb{C}\right)$. Now, observe that $E_{s} \otimes_{\mathbb{C}[G]} \mathbb{C}=0$ for all $s \neq s_{\omega}$, and $E_{s_{\omega}} \otimes_{\mathbb{C}[G]} \mathbb{C}=E_{s_{\omega}}$. Therefore, the multiplication $H_{2}\left(W^{\gamma} ; \mathbb{C}\right) \rightarrow$ $\vartheta_{\omega} H_{2}\left(W^{\gamma} ; \mathbb{C}\right)$ by $\vartheta_{\omega}:=\vartheta_{s_{\omega}}$ induces an isomorphism

$$
H_{2}\left(W^{\gamma} ; \mathbb{C}\right) \otimes_{\mathbb{C}[G]} \mathbb{C} \simeq E_{s_{\omega}} .
$$

Since $\psi^{\omega}(x \otimes u, y \otimes v)=|G| u \bar{v}\left\langle x, \vartheta_{\omega} y\right\rangle=|G| u \bar{v}\left\langle\vartheta_{\omega} x, \vartheta_{\omega} y\right\rangle=|G|\left\langle\vartheta_{\omega} u x, \vartheta_{\omega} v y\right\rangle$ for all $x, y \in H_{2}\left(W^{\gamma} ; \mathbb{C}\right)$ and $u, v \in \mathbb{C}$, the lemma is proved.

If the $q_{i}$ 's are pairwise coprime, then $r_{\omega}: G \rightarrow C_{q}$ is an isomorphism, so $W^{\gamma}=$ $W^{q}$. In this case, $H_{2}\left(W^{\gamma}\right) \otimes_{\mathbb{Z}[G]} \mathbb{C}=H_{2}^{\omega}(W ; \mathbb{C})$, and the forms $\psi^{\omega}$ and $\varphi^{\omega}$ are equal. In general, we have the following result, very much in the spirit of [29].

Lemma 6.3. Let $\omega$ be an element of $T_{\mathbb{Q}}^{\mu}$ and consider $\gamma$ and $s_{\omega}$ such that $\chi_{\omega}=$ $s_{\omega} \circ \gamma$. Then, the Hermitian forms $\varphi^{\omega}$ and $\psi^{\omega}$ are conjugate.

Proof. By the proof of Lemma 6.2 the multiplication by $\vartheta_{\omega}=\vartheta_{s_{\omega}}$ induces an isomorphism

$$
H_{2}\left(W^{\gamma} ; \mathbb{C}\right) \otimes_{\mathbb{C}[G]} \mathbb{C} \rightarrow \vartheta_{\omega} H_{2}\left(W^{\gamma} ; \mathbb{C}\right) \otimes_{\mathbb{C}[G]} \mathbb{C},
$$

so that $\psi^{\omega}$ is conjugate to $|G|\langle$,$\rangle restricted to \vartheta_{\omega} H_{2}\left(W^{\gamma} ; \mathbb{C}\right) \otimes_{\mathbb{C}[G]} \mathbb{C}$ (which is nothing but $\left.E_{s_{\omega}}\right)$. For the same reason, the multiplication by $r_{\omega}\left(\vartheta_{\omega}\right)=\frac{1}{q} \sum_{i=1}^{q} \bar{\alpha}^{i} \tau^{i}$ induces an isomorphism

$$
H_{2}\left(W^{q} ; \mathbb{C}\right) \otimes_{\mathbb{C}\left[C_{q}\right]} \mathbb{C} \rightarrow r_{\omega}\left(\vartheta_{\omega}\right) H_{2}\left(W^{q} ; \mathbb{C}\right) \otimes_{\mathbb{C}\left[C_{q}\right]} \mathbb{C},
$$

so that $\varphi^{\omega}$ is conjugate to $q\langle$,$\rangle restricted to r_{\omega}\left(\vartheta_{\omega}\right) H_{2}\left(W^{q} ; \mathbb{C}\right) \otimes_{\mathbb{C}\left[C_{q}\right]} \mathbb{C}$.

Set $B=\operatorname{ker}\left(s_{\omega}\right)$ and $\mathcal{R}_{G}(B)=\left\{s \in \mathcal{R}_{G} \mid B \subset \operatorname{ker}(s)\right\}$. By [29, p. 205], we have natural isomorphisms of $\mathbb{C}[G]$-modules

$$
H_{2}\left(W^{q} ; \mathbb{C}\right) \simeq\left(\sum_{b \in B} b\right) H_{2}\left(W^{\gamma} ; \mathbb{C}\right) \simeq|B| \bigoplus_{s \in \mathcal{R}_{G}(B)} \vartheta_{s} H_{2}\left(W^{\gamma} ; \mathbb{C}\right),
$$

where $H_{2}\left(W^{q} ; \mathbb{C}\right)$ is a $\mathbb{C}[G]$-module via the homomorphism $\mathbb{C}[G] \rightarrow \mathbb{C}\left[C_{q}\right]$ induced by $r_{\omega}: G \rightarrow C_{q}$. Since $s_{\omega}$ belongs to $\mathcal{R}_{G}(B)$, this gives an isomorphism

$$
r_{\omega}\left(\vartheta_{\omega}\right) H_{2}\left(W^{q} ; \mathbb{C}\right) \simeq|B| \vartheta_{\omega} H_{2}\left(W^{\gamma} ; \mathbb{C}\right) .
$$

Tensoring this isomorphism by $\mathbb{C}$, we see that the intersection form on the space $r_{\omega}\left(\vartheta_{\omega}\right) H_{2}\left(W^{q} ; \mathbb{C}\right) \otimes_{\mathbb{C}\left[C_{q}\right]} \mathbb{C}$ is conjugate to the intersection form on the space $\vartheta_{\omega} H_{2}\left(W^{\gamma} ; \mathbb{C}\right) \otimes_{\mathbb{C}\left[C_{q}\right]} \mathbb{C}$ multiplied by $|B|^{2}$. Summing up everything, $\varphi^{\omega}$ is conjugate to $\psi^{\omega}$ multiplied by $\frac{q|B|^{2}}{|G|}=|B|$. 
6.2. Intersection forms and Seifert forms. For technical reasons, we shall work with branched coverings instead of regular coverings. As above, let $F \subset B^{4}$ be a surface with boundary $L$, and let $W_{F}$ denote its exterior. Given $\omega \in T_{\mathbb{Q}}^{\mu}$, let $q_{i}$ be the order of $\omega_{i}$. The regular covering $W_{F}^{\gamma} \rightarrow W_{F}$ induced by the projection $\gamma: H_{1}\left(W_{F}\right) \rightarrow G=C_{q_{1}} \times \cdots \times C_{q_{\mu}}$ can be extended to a covering $p: \bar{W}_{F}^{\gamma} \rightarrow B^{4}$ branched along $F$, such that $p \mid: p^{-1}\left(F_{i}\right) \rightarrow F_{i}$ is a $G / C_{q_{i}}$-covering branched along $F_{i} \cap F_{j}$ with branch index $q_{j}$. The construction of $\bar{W}_{F}^{\gamma}$ is quite clear except near the intersection points of two components of $F$, where it can be described as follows. An intersection point of $F_{i}$ and $F_{j}$ has a neighborhood $N$ in $B^{4}$ such that the triple $\left(N, F_{i}, F_{j}\right)$ is diffeomorphic to $\left(B^{2} \times B^{2}, B^{2} \times\{0\},\{0\} \times B^{2}\right)$. Then, $p^{-1}(N)$ is a disjoint union of balls, and the covering projection restricted to each of these balls is given by $B^{2} \times B^{2} \rightarrow B^{2} \times B^{2} \simeq N,\left(z_{1}, z_{2}\right) \mapsto\left(e^{2 i \pi / q_{i}} z_{1}, e^{2 i \pi / q_{j}} z_{2}\right)$. Note that the boundary of $\bar{W}_{F}^{\gamma}$ is the covering of $S^{3}$ branched along $L$ induced by the restriction of $\gamma$ to the homology of the exterior of $L$.

Clearly, we can define a twisted intersection form $\bar{\psi}_{F}^{\omega}$ on $H_{2}\left(\bar{W}_{F}^{\gamma}\right) \otimes_{\mathbb{Z}[G]} \mathbb{C}$ as explained in the previous subsection. Furthermore, we have the following result.

Lemma 6.4. The forms $\psi_{F}^{\omega}$ and $\bar{\psi}_{F}^{\omega}$ are conjugate.

Proof. By Lemma 6.2, we just need to check that the inclusion $W_{F}^{\gamma} \subset \bar{W}_{F}^{\gamma}$ induces an isomorphism of $\mathbb{C}$-vector spaces $\vartheta_{\omega} H_{2}\left(W_{F}^{\gamma} ; \mathbb{C}\right) \simeq \vartheta_{\omega} H_{2}\left(\bar{W}_{F}^{\gamma} ; \mathbb{C}\right)$. This follows from a standard Mayer-Vietoris argument (see, e.g. [31, Lemma 5] and [6, Lemma $6.3])$.

This subsection is devoted to the proof of the following proposition, which generalizes [34] and 6, Proposition 6.1]. Note that this result implies that our signatures coincide with invariants introduced by Smolinski 31].

Proposition 6.5. Consider a connected $C$-complex $S \subset S^{3}$ for a $\mu$-colored link L. For $\omega \in T_{\mathbb{Q}}^{\mu}$, let $H(\omega)$ be the corresponding Hermitian matrix (recall Section 2 ). If $F$ is the surface with boundary $L$ obtained by pushing $S$ in $B^{4}$, then $H(\omega)$ is a matrix for $\bar{\psi}_{F}^{\omega}$.

Proof. As the surfaces $S_{i}$ are pushed into $B^{4}$, they trace out 3-manifolds $M_{i}$ homeomorphic to $S_{i} \times[0,1]$ with $L_{i} \times[0,1]$ collapsed to a single copy of $L_{i}$. It may be assumed that the $M_{i}$ 's intersect transversally, so each clasp in $S_{i} \cap S_{j}$ gives a 2-disc in $M_{i} \cap M_{j}$. Now, split $B^{4}$ along $M=M_{1} \cup \cdots \cup M_{\mu}$. The boundary of the resulting manifold $B$ contains two copies $M^{+}$and $M^{-}$of $M$ which intersect along $F$. Let $\{g B\}_{g \in G}$ be disjoint copies of $B$. Consider the 4-dimensional $G$-manifold $E$ obtained by pasting these copies along $g M_{i}^{ \pm} \subset g B$ as follows:

$$
E=\bigsqcup_{g \in G} g B /\left(\tau_{i} g M_{i}^{+}=g M_{i}^{-}\right)_{1 \leq i \leq \mu, g \in G} .
$$

(Recall that $\tau_{i}$ is a preferred generator of $C_{q_{i}}$.) Clearly, the projection $E \rightarrow E / G$ is nothing but the branched covering $\bar{W}_{F}^{\gamma} \rightarrow B^{4}$. Furthermore, there is a retraction by deformation of $B$ onto the cone $C\left(M^{+} \cup M^{-}\right)$over $M^{+} \cup M^{-}$, which itself retracts by deformation on the cone $C F$ over $F$. Therefore, $\bar{W}_{F}^{\gamma}=E$ is homotopy equivalent to the $G$-space

$$
Y=\bigsqcup_{g \in G} g C F /\left(\tau_{i} g F_{i}=g F_{i}\right)_{1 \leq i \leq \mu, g \in G} .
$$


We shall now use this description of $\bar{W}_{F}^{\gamma}$ to compute the $\mathbb{Z}[G]$-module $H_{2}\left(\bar{W}_{F}^{\gamma}\right)=$ $H_{2}(Y)$. Consider a basis of $H_{1}(F)$ whose elements are given by 1-cycles $\left\{e_{\alpha}\right\}_{\alpha \in A}$. For $\alpha \in A$, let $I(\alpha)$ denote the set of indices $i$ such that $e_{\alpha}$ meets $F_{i}$. Given such an $e_{\alpha}$ in $F$, let $C e_{\alpha} \subset C F$ denote the cone over $e_{\alpha}$, and $\Sigma e_{\alpha}$ the 'suspension' defined by

$$
\Sigma e_{\alpha}=\prod_{i \in I(\alpha)}\left(1-\tau_{i}\right) C e_{\alpha} \subset Y .
$$

By construction of $Y, \Sigma e_{\alpha}$ is a 2-cycle in $Y$. Furthermore, a massive use of the Mayer-Vietoris exact sequence leads to the following fact: the $\mathbb{Z}[G]$-module $H_{2}(Y)$ is given by

$$
H_{2}(Y)=\bigoplus_{\alpha \in A}\left(\mathbb{Z}[G] /\left(1+\tau_{i}+\cdots+\tau_{i}^{q_{i}-1}\right)_{i \in I(\alpha)}\right) \Sigma e_{\alpha} .
$$

Using the fact that $\omega_{i} \neq 1$ for $i \in I(\alpha)$, we have

$$
\begin{aligned}
H_{2}(Y) \otimes_{\mathbb{Z}[G]} \mathbb{C} & =\bigoplus_{\alpha \in A}\left(\left(\mathbb{Z}[G] /\left(1+\tau_{i}+\cdots+\tau_{i}^{q_{i}-1}\right)_{i \in I(\alpha)}\right) \otimes_{\mathbb{Z}[G]} \mathbb{C}\right) \Sigma e_{\alpha} \\
& =\bigoplus_{\alpha \in A} \mathbb{C} \Sigma e_{\alpha} .
\end{aligned}
$$

Furthermore, since $\omega_{i} \neq 1$ for $i \notin I(\alpha), x_{\alpha}=\prod_{i=1}^{\mu}\left(1-\tau_{i}\right) C e_{\alpha}$ is a non-zero multiple of $\Sigma e_{\alpha}$ in $H_{2}(Y) \otimes_{\mathbb{Z}[G]} \mathbb{C}$. Therefore, a basis of the latter space is given by $\left\{x_{\alpha}\right\}_{\alpha \in A}$.

We are left with the computation of the form $\bar{\psi}_{F}^{\omega}$ on the elements of this basis. To do this, we shall deform $x_{\alpha}$ into another 2 -cycle $\widetilde{x}_{\alpha}$ as follows. First, note that

$$
x_{\alpha}=\sum_{\varepsilon} \varepsilon_{1} \cdots \varepsilon_{\mu} \tau_{1}^{\frac{1-\varepsilon_{1}}{2}} \cdots \tau_{\mu}^{\frac{1-\varepsilon_{\mu}}{2}} C e_{\alpha}
$$

where the sum is on all sequences $\varepsilon=\left(\varepsilon_{1}, \ldots, \varepsilon_{\mu}\right)$ of \pm 1 's. Set

$$
\widetilde{x}_{\alpha}=\sum_{\varepsilon} \varepsilon_{1} \cdots \varepsilon_{\mu} \tau_{1}^{\frac{1-\varepsilon_{1}}{2}} \cdots \tau_{\mu}^{\frac{1-\varepsilon_{\mu}}{2}} C\left(i^{\varepsilon}\left(e_{\alpha}^{\prime}\right)\right),
$$

where $e_{\alpha}^{\prime}$ is the 1-cycle in $S$ corresponding to $e_{\alpha}$ in $F$, and $i^{\varepsilon}$ is the map defined in Section 2. Clearly, $\widetilde{x}_{\alpha}$ is a 2-cycle and $\left\langle\widetilde{x}_{\alpha}, g x_{\beta}\right\rangle=\left\langle x_{\alpha}, g x_{\beta}\right\rangle$ for all $g \in G$ and $\beta \in A$. Furthermore, the intersection number between $C\left(i^{\varepsilon}\left(e_{\alpha}^{\prime}\right)\right)$ and $g C e_{\beta}$ is given by

$$
C\left(i^{\varepsilon}\left(e_{\alpha}^{\prime}\right)\right) \cdot\left(g C e_{\beta}\right)= \begin{cases}l k\left(i^{\varepsilon}\left(e_{\alpha}^{\prime}\right), e_{\beta}^{\prime}\right) & \text { if } g=1, \\ 0 & \text { else. }\end{cases}
$$

Since $G$ acts by isometries, this is sufficient in order to determine $\bar{\psi}_{F}^{\omega}\left(x_{\alpha}, x_{\beta}\right)=$ $\sum_{g \in G}\left\langle\widetilde{x}_{\alpha}, g x_{\beta}\right\rangle s_{\omega}(g)$. The result of this tedious computation is

$$
\bar{\psi}_{F}^{\omega}\left(x_{\alpha}, x_{\beta}\right)=\prod_{i=1}^{\mu}\left(1-\bar{\omega}_{i}\right) \sum_{\varepsilon} \varepsilon_{1} \cdots \varepsilon_{\mu} \omega_{1}^{\frac{1-\varepsilon_{1}}{2}} \cdots \omega_{\mu}^{\frac{1-\varepsilon_{\mu}}{2}} l k\left(i^{\varepsilon}\left(e_{\alpha}^{\prime}\right), e_{\beta}^{\prime}\right),
$$

concluding the proof. 
6.3. Proof of Theorem 6.1. We need one more result.

Proposition 6.6. For all $\omega$ in $T_{\mathbb{Q}}^{\mu}$, sign $\left(\varphi_{F}^{\omega}\right)$ does not depend on the choice of the surface $F$ in $B^{4}$ with boundary $L$.

Proof. By Lemmas 6.2, 6.3 and 6.4 $\varphi_{F}^{\omega}$ is conjugate to the restriction to $E_{s_{\omega}}$ of the intersection form on $H_{2}\left(\bar{W}_{F}^{\gamma} ; \mathbb{C}\right)$. Moreover, the signature of the latter form is easily seen to be given by a linear combination of the $g$-signatures of $\bar{W}_{F}^{\gamma}$, denoted by $\sigma\left(g, \bar{W}_{F}^{\gamma}\right)$ (see [15]). So, we are left with the proof that $\sigma\left(g, \bar{W}_{F}^{\gamma}\right)$ does not depend on the choice of $F$. Consider two surfaces $F$ and $F^{\prime}$ with boundary $L$, and glue together $\bar{W}_{F}^{\gamma}$ and $-\bar{W}_{F^{\prime}}^{\gamma}$ along their common boundary. By Novikov additivity, the resulting manifold $Z$ satisfies $\sigma(g, Z)=\sigma\left(g, \bar{W}_{F}^{\gamma}\right)-\sigma\left(g, \bar{W}_{F^{\prime}}^{\gamma}\right)$. Using the AtiyahSinger $G$-signature theorem [1], one easily checks that $\sigma(g, Z)$ is zero for all $g \in G$ (see [31, p. 216]). This completes the proof.

Proof of Theorem 6.1. The equality $\sigma_{L}(\omega)=\operatorname{sign}\left(\varphi_{F}^{\omega}\right)$ is a direct consequence of Lemma 6.3. Lemma 6.4. Proposition 6.5 and Proposition 6.6. On the other hand, consider the exact sequence of the pair $\left(W_{F}, \partial W_{F}\right)$ with (twisted) complex coefficients. Clearly, a matrix for $\varphi_{F}^{\omega}$ is (the transpose of) a matrix for $H_{2}^{\omega}\left(W_{F} ; \mathbb{C}\right) \rightarrow H_{2}^{\omega}\left(W_{F}, \partial W_{F} ; \mathbb{C}\right)$. This leads to

$$
\operatorname{null}\left(\varphi_{F}^{\omega}\right)=\operatorname{dim} H_{1}^{\omega}\left(\partial W_{F} ; \mathbb{C}\right)-\operatorname{dim} H_{1}^{\omega}\left(W_{F} ; \mathbb{C}\right)+\operatorname{dim} H_{1}^{\omega}\left(W_{F}, \partial W_{F} ; \mathbb{C}\right) .
$$

By duality, $\operatorname{dim} H_{1}^{\omega}\left(W_{F}, \partial W_{F} ; \mathbb{C}\right)=\operatorname{dim} H_{3}^{\omega}\left(W_{F} ; \mathbb{C}\right)$. Finally, a standard MayerVietoris argument shows that the inclusion $X \subset \partial W_{F}$ induces an isomorphism $H_{1}^{\omega}(X ; \mathbb{C})=H_{1}^{\omega}\left(\partial W_{F} ; \mathbb{C}\right)$. This gives

$$
\operatorname{dim} H_{1}^{\omega}(X ; \mathbb{C})=\operatorname{null}\left(\varphi_{F}^{\omega}\right)+\operatorname{dim} H_{1}^{\omega}\left(W_{F} ; \mathbb{C}\right)-\operatorname{dim} H_{3}^{\omega}\left(W_{F} ; \mathbb{C}\right) .
$$

Note that this equation holds for any surface $F$ in $B^{4}$ with boundary $L$.

We now turn to the proof of the equality $\eta_{L}(\omega)=\operatorname{dim} H_{1}^{\omega}(X ; \mathbb{C})$, assuming the notation of the previous subsections. Let $F$ be a connected C-complex for $L$ pushed in $B^{4}$. By Lemma 6.3. Lemma 6.4 and Proposition 6.5. $\eta_{L}(\omega)=\operatorname{null}\left(\varphi_{F}^{\omega}\right)$, so we are left with the proof that $H_{1}^{\omega}\left(W_{F} ; \mathbb{C}\right)=H_{3}^{\omega}\left(W_{F} ; \mathbb{C}\right)=0$ for such an $F$. By the proof of Lemma 6.3. $H_{*}^{\omega}\left(W_{F} ; \mathbb{C}\right)=H_{*}\left(W_{F}^{\gamma}\right) \otimes_{\mathbb{Z}[G]} \mathbb{C}$. Furthermore, a Mayer-Vietoris argument shows that $H_{*}\left(W_{F}^{\gamma}\right)=H_{*}\left(\bar{W}_{F}^{\gamma}\right)$. Finally, recall that $\bar{W}_{F}^{\gamma}$ has the homotopy type of a $G$-space obtained by gluing cones over $F$ along their boundary (see the proof of Proposition 6.5). Since $F$ is connected and has no closed component, $H_{1}\left(\bar{W}_{F}^{\gamma}\right)=H_{3}\left(\bar{W}_{F}^{\gamma}\right)=0$. This concludes the proof.

This 4-dimensional interpretation of $\sigma_{L}(\omega)$ can be used to give an alternative proof (for $\omega \in T_{\mathbb{Q}}^{\mu}$ ) of several results obtained in Section 2 . For example, Propositions 6.5 and 6.6 imply the independence of $\sigma_{L}(\omega)$ on the C-complex (Theorem 2.1).

As promised in Section 2, we shall now give another proof of Proposition 2.5. Consider $\omega=\left(\omega_{1}, \ldots, \omega_{\mu}, \omega_{\mu}\right) \in T_{\mathbb{Q}}^{\mu+1}$, and let $F=F_{1} \cup \cdots \cup F_{\mu} \cup F_{\mu+1}$ be a surface in $B^{4}$ with boundary $L$. In a closed neighborhood of an intersection point of $F_{\mu}$ and $F_{\mu+1}, F$ is given by two transversal disks with boundary a 2-colored Hopf link. Let $F^{\prime}=F_{1}^{\prime} \cup \cdots \cup F_{\mu}^{\prime}$ be the surface obtained by replacing these disks by a cylinder, whose boundary is a 1-colored Hopf link. Clearly, $F^{\prime}$ has boundary the $\mu$-colored link $L^{\prime}$. Note that the signature of a 2-colored Hopf link is zero, while the signature of the positive (resp. negative) 1-colored Hopf link is -1 (resp. +1 ). 
By the additivity of the signature, these local transformations decrease $\sigma_{L}(\omega)$ by the algebraic intersection number $F_{\mu} \cdot F_{\mu+1}=l k\left(L_{\mu}, L_{\mu+1}\right)$.

6.4. On the Casson-Gordon invariants of 3-manifolds. Let $M$ be an oriented closed 3-manifold, and let $\chi: H_{1}(M) \rightarrow \mathbb{C}^{*}$ be a character of finite order. The following reformulation of the Atiyah-Singer invariant [1] of $(M, \chi)$ is due to Casson and Gordon [2, 3. Since $\chi$ is of finite order, its image is the cyclic subgroup of $\mathbb{C}^{*}$ generated by $\alpha=e^{2 i \pi / q}$, for some $q$. It induces a $q$-fold cyclic covering $M^{q} \rightarrow M$ with a canonical deck transformation $\tau$ generating the group $C_{q}$ of the covering. Since the bordism group $\Omega_{3}\left(B C_{q}\right)$ is equal to $C_{q}$, there is a positive integer $n$ such that $n$ disjoint copies of $M$ bound a compact oriented 4-manifold $W$ over $B C_{q}$. Let $W^{q} \rightarrow W$ be the induced $q$-fold covering. The deck transformation $\tau$ extends to a deck transformation of $W^{q}$, also denoted by $\tau$. As above, let $H_{*}^{\chi}(W ; \mathbb{C})$ denote the homology of the chain complex $C_{*}\left(W^{q}\right) \otimes_{\mathbb{Z}\left[C_{q}\right]} \mathbb{C}$, where the structure of $\mathbb{Z}\left[C_{q}\right]$ module on $\mathbb{C}$ is given by the map $C_{q} \rightarrow \mathbb{C}^{*}$ which sends $\tau$ to $\alpha=e^{2 i \pi / q}$. Finally, let $\varphi^{\chi}$ be the twisted intersection form on $H_{2}^{\chi}(W ; \mathbb{C})$.

Definition. The Casson-Gordon invariant of $(M, \chi)$ is

$$
\sigma(M, \chi)=\frac{1}{n}\left(\operatorname{sign}\left(\varphi^{\chi}\right)-\operatorname{sign}(W)\right) .
$$

The related nullity is defined by $\eta(M, \chi)=\operatorname{dim} H_{1}^{\chi}(M ; \mathbb{C})$.

The fact that $\sigma(M, \chi)$ depends only on the pair $(M, \chi)$ is a consequence of the Atiyah-Singer $G$-signature theorem, and Novikov additivity. Note that one may also consider branched coverings in order to define or compute this invariant (see, e.g. [12, Proposition 3.5]).

The aim of this subsection is to relate the Casson-Gordon invariant of a manifold obtained by surgery on a framed link $L$ to the signature $\sigma_{L}$ of this link. We have the following result.

Theorem 6.7. Let $M$ be the 3-manifold obtained by surgery on a framed link $L$ with $\nu$ components and linking matrix $\Lambda$. Let $\chi: H_{1}(M) \rightarrow \mathbb{C}^{*}$ be the character mapping the meridian of the $i^{\text {th }}$ component of $L$ to $\alpha^{n_{i}}$, where $\alpha=e^{2 i \pi / q}$ and $n_{i}$ is an integer coprime to $q$. Consider $L$ as a $\nu$-colored link and set $\omega=\left(\alpha^{n_{1}}, \ldots, \alpha^{n_{\nu}}\right)$. Then,

$$
\begin{aligned}
\sigma(M, \chi) & =\left(\sigma_{L}(\omega)-\sum_{i<j} \Lambda_{i j}\right)-\operatorname{sign}(\Lambda)+\frac{2}{q^{2}} \sum_{i, j}\left(q-n_{i}\right) n_{j} \Lambda_{i j}, \\
\eta(M, \chi) & =\eta_{L}(\omega) .
\end{aligned}
$$

Note that if all the $n_{i}$ 's are equal, then this formula together with Proposition 2.5 give back Casson and Gordon's [3, Lemma 3.1]. See also Gilmer 12, Theorem 3.6]. On the other hand, if the matrix $\Lambda$ is zero, then $\sigma_{L}(\omega)=\sigma(M, \chi)$. Hence our signature extends (a special case of) the invariant introduced by Levine in 21] for links with $\Lambda_{i j}=0$ for all $i \neq j$. This also relates $\sigma_{L}(\omega)$ with link signatures of Gilmer [12].

Let us point out an interesting feature of this result before giving its proof. Recall that all the signatures $\sigma_{L}(\omega)$ of a fixed colored link $L$ are given by the signature of a single matrix $H$ evaluated at $\omega$. Using Theorem 6.7, one can compute the Casson-Gordon invariants of all 3-manifolds obtained by surgery on $L$, for many characters of finite order, by using this single matrix. 
Proof. Let $F=F_{1} \cup \cdots \cup F_{\nu}$ be the surface obtained by pushing a connected $C$ complex for $L$ in $B^{4}$. Denote by $\left\{p_{\ell}\right\}_{\ell}$ the finite set of double points of $F$ (coming from the clasps of the C-complex) and by $\left\{B_{\ell}\right\}_{\ell}$ a set of small disjoint closed 4-balls such that $p_{\ell} \in$ int $B_{\ell}$ for all $\ell$. We shall denote by $F_{j(\ell)}$ and $F_{k(\ell)}$ the components of $F$ which intersect at $p_{\ell}$. Note that $F \cap B_{\ell}$ consists of two transverse discs with boundary a Hopf link $K_{\ell} \subset S_{\ell}^{3}=\partial B_{\ell}$. Let $\epsilon_{\ell}= \pm 1$ denote the linking number of the components of $K_{\ell}$ (that is, the algebraic intersection number of $F_{j(\ell)}$ and $F_{k(\ell)}$ at $\left.p_{\ell}\right)$. Set $\Omega=B^{4} \backslash \bigsqcup_{\ell}$ int $B_{\ell}$. Clearly, the character $\chi$ restricted to the exterior of $L$ in $S^{3}$ extends to a character on the exterior of $F$ in $B^{4}$, which itself restricts to the exterior of $K_{\ell}$ in $S_{\ell}^{3}$. This restriction maps the meridians of $K_{\ell}$ to $\chi\left(m_{j(\ell)}\right)=\alpha^{n_{j(\ell)}}$ and $\chi\left(m_{k(\ell)}\right)=\alpha^{n_{k(\ell)}}$.

Now, let $U$ be the 4-manifold obtained by attaching 2-handles $B^{2} \times B^{2}$ to $\Omega$ as follows. First, attach 2-handles to $\Omega$ along a tubular neighborhood of $L \subset S^{3}$ according to the framings $\left\{\Lambda_{i i}\right\}_{i}$. Then, for all $\ell$, perform a surgery along $K_{\ell} \subset S_{\ell}^{3}$ according to framings $f_{j(\ell)}^{\ell}$ and $f_{k(\ell)}^{\ell}$ which satisfy the following property: the character on the exterior of $K_{\ell}$ in $S_{\ell}^{3}$ extends to the 3-manifold $M_{\ell}$ obtained by surgery on the framed link $K_{\ell}$. This is the case if and only if the congruences $f_{j(\ell)}^{\ell} n_{j(\ell)}+\epsilon_{\ell} n_{k(\ell)} \equiv \epsilon_{\ell} n_{j(\ell)}+f_{k(\ell)}^{\ell} n_{k(\ell)} \equiv 0(\bmod q)$ hold. By hypothesis, $n_{j(\ell)}$ and $n_{k(\ell)}$ are invertible modulo $q$, so such framings exist.

Let $F^{\prime} \subset$ int $U$ be the smooth closed surface with $\nu$ connected components obtained from $F \cap \Omega$ by gluing the cores of the 2-handles. Let $U_{F^{\prime}}$ be the exterior of $F^{\prime}$ in $U$. From $\chi$ (that is, from $\omega$ ), one easily constructs a character on $H_{1}\left(U_{F^{\prime}}\right)$ inducing a twisted intersection form $\varphi^{\omega}$ on $H_{2}^{\omega}\left(U_{F^{\prime}} ; \mathbb{C}\right)$. Since $\partial U=M \sqcup \bigsqcup_{\ell}\left(-M_{\ell}\right)$, Gilmer's [12, Proposition 3.5] gives

$$
\sigma(M, \chi)-\sum_{\ell} \sigma\left(M_{\ell}, \chi\right)=\operatorname{sign}\left(\varphi^{\omega}\right)-\operatorname{sign}(U)+\frac{2}{q^{2}} \sum_{i=1}^{\nu}\left(q-n_{i}\right) n_{i}\left(F_{i}^{\prime} \cdot F_{i}^{\prime}\right) .
$$

(Note that a Mayer-Vietoris argument shows that $\varphi^{\omega}$ is conjugate to the form related to the covering of $U$ branched along $F^{\prime}$, considered by Gilmer in his formula.) We shall now compute separately each term of this equation.

Recall that $\omega$ induces a $C_{q} \times \cdots \times C_{q}$-covering of $B^{4}$ branched along $F$. By Proposition 6.5. $\sigma_{L}(\omega)$ is equal to the signature of the corresponding intersection form $\bar{\psi}_{F}^{\omega}$. Furthermore, the signature corresponding to the covering of $B_{\ell}$ branched along $F \cap B_{\ell}$ is equal to zero. Therefore, Novikov additivity implies that $\operatorname{sign}\left(\bar{\psi}_{F}^{\omega}\right)$ is equal to the signature of the twisted intersection form of the covering of $\Omega$ branched along $F \cap \Omega$. Finally, a standard Mayer-Vietoris argument shows that adding 2handles to a 4-manifold has no effect on its twisted signature, so

$$
\operatorname{sign}\left(\varphi^{\omega}\right)=\sigma_{L}(\omega)
$$

One easily checks that a matrix for the intersection form on $H_{2}(U)$ is given by $\Lambda \oplus \bigoplus_{\ell}\left(-\Lambda_{\ell}\right)$, where $\Lambda_{\ell}$ is the linking matrix of $K_{\ell}$. Therefore,

$$
\operatorname{sign}(U)=\operatorname{sign}(\Lambda)-\sum_{\ell} \operatorname{sign}\left(\Lambda_{\ell}\right) .
$$

Using [12, Proposition 3.8 and p. 367], we obtain $\sigma\left(M_{\ell}, \chi\right)=-\epsilon_{\ell}-\operatorname{sign}\left(\Lambda_{\ell}\right)+$ $\frac{2}{q^{2}} s_{\ell}$, where the integer $s_{\ell}$ is given by

$s_{\ell}=\left(q-n_{j(\ell)}\right) n_{j(\ell)} f_{j(\ell)}^{\ell}+\left(q-n_{k(\ell)}\right) n_{k(\ell)} f_{k(\ell)}^{\ell}+\epsilon_{\ell}\left(\left(q-n_{j(\ell)}\right) n_{k(\ell)}+\left(q-n_{k(\ell)}\right) n_{j(\ell)}\right)$. 
Finally, $F_{i}^{\prime} \cdot F_{i}^{\prime}=\Lambda_{i i}-\sum_{\ell} f_{i}^{\ell}$, where the sum is on all indices $\ell$ such that $j(\ell)=i$ or $k(\ell)=i$. The first equality of the theorem now follows from the fact that $\sum_{\ell} \epsilon_{\ell}=\sum_{i<j} \Lambda_{i j}$.

Since $M$ is obtained from the link complement $X$ by adjoining tori, an easy Mayer-Vietoris argument gives the equality between the nullities.

\section{Concordance And EXIstence of SURFACES in $B^{4}$}

The properties of $\sigma_{L}(\omega)$ and $\eta_{L}(\omega)$ studied in this section do not hold for all $\omega$ in $T_{*}^{\mu}$. We shall denote by $T_{\mathcal{P}}^{\mu}$ the dense subset of $T_{*}^{\mu}$ constituted by the elements $\omega=\left(\omega_{1}, \ldots, \omega_{\mu}\right)$ which satisfy the following condition: there exists a prime $p$ such that for all $i$, the order of $\omega_{i}$ is a power of $p$.

We first prove the invariance of the restriction of $\sigma_{L}$ and $\eta_{L}$ to $T_{\mathcal{P}}^{\mu}$ under (colored) concordance. Then, we show that the signature and nullity provide a lower bound for the genus of a surface in $B^{4}$ with boundary $L$ (Theorem[7.2). Finally subsection 7.2 deals with an analogous result concerning surfaces in $S^{4}$ whose intersection with a standardly embedded 3 -sphere is equal to the colored link $L$. These results generalize celebrated theorems of Murasugi-Tristram and Kauffman-Taylor.

Definition. Two colored links $L$ and $L^{\prime}$ with $\nu$ components are said to be concordant if there exists a collection of smooth disjoint cylinders $T_{1}, \ldots, T_{\nu}$ properly embedded in $S^{3} \times[0,1]$, such that for all $i, T_{i}$ is a concordance between components of $L$ and $L^{\prime}$ of the same color.

Theorem 7.1. For all $\omega \in T_{\mathcal{P}}^{\mu}, \sigma_{L}(\omega)$ and $\eta_{L}(\omega)$ are concordance invariants.

This result follows from the fact that the exterior of the concordance is a homology cobordism. The detailed proof can be found in [9, Theorem 4.15] for the case of colored links with $l k\left(L_{i}, L_{j}\right)=0$ for all $i \neq j$. It obviously extends to the general case. Note that this theorem can also be viewed as a consequence of [14, Theorem 9].

\subsection{Surfaces with double points and Murasugi-Tristram inequality.}

Theorem 7.2. Suppose that $F=F_{1} \cup \cdots \cup F_{\mu}$ in $B^{4}$ has boundary $L$ (in the sense of Section 6). Set $\beta_{1}=\sum_{i} r k H_{1}\left(F_{i}\right)$, and let $c$ be the number of double points of $F$. Then, for all $\omega \in T_{\mathcal{P}}^{\mu}$,

$$
\left|\sigma_{L}(\omega)\right|+\left|\eta_{L}(\omega)-\mu+1\right| \leq \beta_{1}+c .
$$

The case $c=0$ can be found in [9, Theorem 5.19]. The proof of this generalization is very similar. We refer to the upcoming paper [27] for an interesting application of this result to the study of real algebraic plane curves.

Proof. Let $W_{F}$ denote the exterior of $F$ in $B^{4}$. The non-vanishing homology groups of $W_{F}$ are given by

$$
H_{0}\left(W_{F}\right)=\mathbb{Z}, H_{1}\left(W_{F}\right)=\mathbb{Z}^{\mu} \text { and } H_{2}\left(W_{F}\right)=\mathbb{Z}^{\beta_{1}+c} .
$$

Set $\beta_{k}^{\omega}=\operatorname{dim} H_{k}^{\omega}\left(W_{F}, \mathbb{C}\right)$. Clearly, $\beta_{0}^{\omega}=\beta_{4}^{\omega}=0$. Since $\omega$ belongs to $T_{\mathcal{P}}^{\mu}$, the order of the associated cyclic covering of $W_{F}$ is a power of prime. Therefore, we can make use of Gilmer's results [12. In particular, by [12, Proposition 1.4], $\beta_{3}^{\omega}=0$. It follows that

$$
\beta_{2}^{\omega}-\beta_{1}^{\omega}=\chi\left(W_{F}\right)=1-\mu+\beta_{1}+c
$$


Therefore,

$$
\left|\operatorname{sign}\left(\varphi^{\omega}\right)\right|+\operatorname{null}\left(\varphi^{\omega}\right) \leq \beta_{2}^{\omega}=\beta_{1}^{\omega}+1-\mu+\beta_{1}+c .
$$

By Theorem 6.1, sign $\left(\varphi^{\omega}\right)=\sigma_{L}(\omega)$ and null $\left(\varphi^{\omega}\right)+\beta_{1}^{\omega}=\eta_{L}(\omega)$, leading to

$$
\left|\sigma_{L}(\omega)\right|+\eta_{L}(\omega) \leq 2 \beta_{1}^{\omega}+1-\mu+\beta_{1}+c .
$$

By [12, Proposition 1.5], $\beta_{1}^{\omega} \leq \mu-1$, so $\left|\sigma_{L}(\omega)\right|+\eta_{L}(\omega)-\mu+1 \leq \beta_{1}+c$, giving the first part of the inequality. On the other hand, $\beta_{1}^{\omega}=\eta_{L}(\omega)-\operatorname{null} \varphi^{\omega} \leq \eta_{L}(\omega)$, so $\left|\sigma_{L}(\omega)\right|-\eta_{L}(\omega)+\mu-1 \leq \beta_{1}+c$, completing the proof of the theorem.

\subsection{The slice genus.}

Definition. Let $S^{3}$ denote the standard embedding of the 3 -sphere in $S^{4}$. The slice genus $g_{s}(L)$ of a $\mu$-colored link $L$ is the minimal genus of a closed oriented smooth surface $P=P_{1} \sqcup \cdots \sqcup P_{\mu} \subset S^{4}$ such that $P_{i} \cap S^{3}=L_{i}$ for all $i$. A $\mu$-colored link is said to be slice if its slice genus is zero.

Note that for such a surface to exist, we must have $l k\left(L_{i}, L_{j}\right)=0$ for all $i \neq j$. This definition should be understood as a unification of several well-known notions of 'sliceness.' Indeed, consider the case $\mu=1$. A 1-colored link is slice if it is the cross-section of a smooth 2-sphere in $S^{4}$, that is, using Fox's terminology [11, if it is slice in the ordinary sense. On the other hand, consider a $\nu$-colored link with $\nu$ components. Such a colored link is slice if it is the cross-section of $\nu$ smooth disjoint 2 -spheres in $S^{4}$. According to Fox, such a link is called slice in the strong sense.

The signature and nullity provide a lower bound for the slice genus of a colored link. Indeed, we have the following generalization of [18, Theorem 3.13].

Theorem 7.3. For all $\omega$ in $T_{\mathcal{P}}^{\mu}$,

$$
\left|\sigma_{L}(\omega)\right| \leq g_{s}(L)+\min \left(0, \eta_{L}(\omega)+1-\mu\right) .
$$

Proof. Consider a closed oriented smooth surface $P=P_{1} \sqcup \cdots \sqcup P_{\mu}$ in $S^{4}$ such that $P_{i} \cap S^{3}=L_{i}$ for all $i$. It may be assumed that each $P_{i}$ is connected. Let $W$ be the exterior of $P$ in $S^{4}$. By duality, the homology of $W$ is given by

$$
H_{0}(W)=\mathbb{Z}, H_{1}(W)=\mathbb{Z}^{\mu}, H_{2}(W)=\mathbb{Z}^{2 g} \text { and } H_{3}(W)=\mathbb{Z}^{\mu},
$$

where $g$ denotes the genus of $P$. As in the previous section, any $\omega$ in $T_{\mathcal{P}}^{\mu}$ induces a character $\chi_{\omega}: H_{1}(W) \rightarrow \mathbb{C}^{*}$ of prime power order sending the meridian of $P_{i}$ to $\omega_{i}$. For simplicity, we simply write $H_{*}^{\omega}(W)$ for $H_{*}^{\omega}(W ; \mathbb{C})$.

Let $X$ be the exterior of $L$ in $S^{3}$. The sphere $S^{3}$ standardly embedded in $S^{4}$ splits $P$ into two surfaces $F_{1}$ and $F_{2}$ with $F_{1} \cap F_{2}=L$. The manifold $W$ can be described as a union $W_{1} \cup W_{2}$ with $W_{1} \cap W_{2}=X$, where $W_{i}$ is the complement of an open tubular neighborood of $F_{i}$ in $B^{4}$ for $i=1,2$. The character $\chi_{\omega}$ restricts to characters on $H_{1}\left(W_{i}\right)$. Let $\varphi_{i}^{\omega}$ be the intersection form on the corresponding twisted homology $H_{2}^{\omega}\left(W_{i}\right)$. By Theorem 6.1 $\sigma_{L}(\omega)=\operatorname{sign}\left(\varphi_{i}^{\omega}\right)$ for $i=1,2$. Clearly, $\varphi_{i}^{\omega}$ is dual to the inclusion homomorphism $j_{i}: H_{2}^{\omega}\left(W_{i}\right) \rightarrow H_{2}^{\omega}\left(W_{i}, M_{i}\right)$, where $M_{i}$ stands for $\partial W_{i}$. It follows that $\left|\sigma_{L}(\omega)\right| \leq \operatorname{dim} K_{i}$, where $K_{i}=H_{2}^{\omega}\left(W_{i}\right) / \operatorname{ker}\left(j_{i}\right)$.

By the Mayer-Vietoris exact sequence with twisted coefficients, we have an isomorphism

$$
H_{2}^{\omega}\left(W_{1}, M_{1}\right) \oplus H_{2}^{\omega}\left(W_{2}, M_{2}\right) \simeq H_{2}^{\omega}\left(W, M_{1} \cup M_{2}\right)
$$


which fits into the following commutative diagram, where the lines are exact:

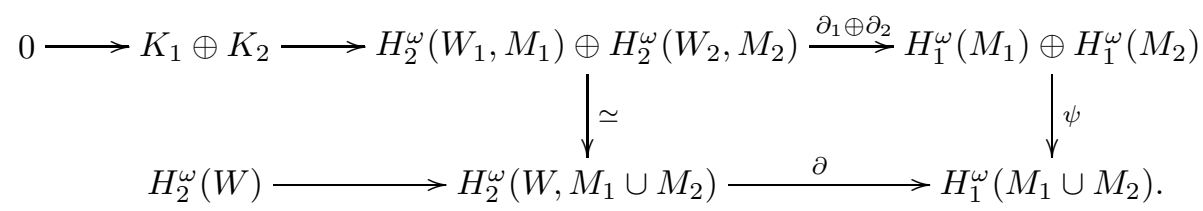

Therefore, $K_{1} \oplus K_{2}=\operatorname{Ker}\left(\partial_{1} \oplus \partial_{2}\right) \subset \operatorname{Ker}\left(\psi \circ\left(\partial_{1} \oplus \partial_{2}\right)\right) \simeq \operatorname{Ker}(\partial)$. This implies that $\operatorname{dim} K_{1}+\operatorname{dim} K_{2} \leq \beta_{2}^{\omega}$, so $2\left|\sigma_{L}(\omega)\right| \leq \beta_{2}^{\omega}$. The equation

$$
1-2 \mu+2 g=\chi(W)=-\beta_{1}^{\omega}+\beta_{2}^{\omega}-\beta_{3}^{\omega}
$$

leads to

$$
2\left|\sigma_{L}(\omega)\right| \leq 1-2 \mu+2 g+\beta_{1}^{\omega}+\beta_{3}^{\omega} .
$$

By [12, Propositions 1.4 and 1.5], $\beta_{3}^{\omega} \leq \operatorname{dim} H_{3}(W)=\mu$ and $\beta_{1}^{\omega}(W) \leq \operatorname{dim} H_{1}(W)$ $-1=\mu-1$. It follows that $\left|\sigma_{L}(\omega)\right| \leq g$ and the first part of the inequality is proved.

To check the second part, let us denote by $\mu_{i}$ the number of components of $F_{i}$ and by $\beta_{1}\left(F_{i}\right)$ the rank of $H_{1}\left(F_{i}\right)$. Since $P=F_{1} \cup F_{2}$ and $F_{1} \cap F_{2}=L$, the additivity of the Euler characteristic implies

$$
\mu_{1}+\mu_{2}-\beta_{1}\left(F_{1}\right)-\beta_{1}\left(F_{2}\right)=2 \mu-2 g .
$$

We then apply Theorem 7.2 to the surfaces $F_{1}$ and $F_{2}$, giving

$$
\left|\sigma_{L}(\omega)\right|-\eta_{L}(\omega)+\mu_{i}-1 \leq \beta_{1}\left(F_{i}\right),
$$

for $i=1,2$. These three equations easily give

$$
\left|\sigma_{L}(\omega)\right| \leq g+\eta_{L}(\omega)+1-\mu,
$$

which implies the second part of the inequality.

Corollary 7.4. If a $\mu$-colored link $L$ is slice, then $\sigma_{L}(\omega)=0$ and $\eta_{L}(\omega) \geq \mu-1$ for all $\omega$ in $T_{\mathcal{P}}^{\mu}$.

The case $\mu=1$ gives the following result.

Corollary 7.5. If a link is slice in the ordinary sense, then $\sigma_{L}(\omega)=0$ for all $\omega$ roots of the unity of prime power order.

On the other hand, if $\mu$ is the maximum number of colors, we get:

Corollary 7.6. If a link with $\nu$ components is slice in the strong sense, then $\sigma_{L}\left(\omega_{1}, \ldots, \omega_{\nu}\right)=0$ and $\eta_{L}\left(\omega_{1}, \ldots, \omega_{\nu}\right) \geq \nu-1$ for all $\left(\omega_{1}, \ldots, \omega_{\nu}\right) \in T_{\mathcal{P}}^{\nu}$.

Finally, let $\Sigma_{L}$ denote the zero in $T_{*}^{\mu}$ of the first non-vanishing Alexander ideal of $L$. By Theorem 4.1 the signature and nullity of $L$ are continuous functions on $T_{*}^{\mu} \backslash \Sigma_{L}$. By density of $T_{\mathcal{P}}^{\mu}$, all the results stated in this section hold for $\omega$ in $T_{*}^{\mu} \backslash \Sigma_{L}$.

Let us conclude this paper with one last didactic example.

Example 7.7. In [11, Fox presents the link illustrated in Figure 20, It is a very simple link which is slice in the ordinary sense, but not in the strong sense. We shall compute the signatures of this link in order to test the results of this section. Let us order its components as illustrated to obtain a 3 -colored link $L$. There is an obvious C-complex for $L$ which has the homotopy type of a circle. The corresponding Seifert 


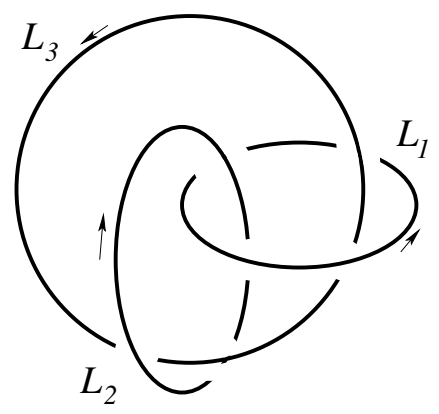

FiguRE 20.

matrices are given by $A^{\varepsilon}=(-1)$ if $\pm \varepsilon=(1,-1,1)$, and $A^{\varepsilon}=(0)$ else. Therefore, we obtain

$$
\sigma_{L}\left(\omega_{1}, \omega_{2}, \omega_{3}\right)=\operatorname{sgn} \Re\left(\left(\omega_{1}-1\right)\left(\bar{\omega}_{2}-1\right)\left(\omega_{3}-1\right)\right),
$$

where $\operatorname{sgn}$ denotes the sign function. By Corollary 7.6, the 3-colored link $L$ is not slice in the strong sense (which is obvious since the linking numbers don't vanish). On the other hand, Proposition 2.5 implies that the Levine-Tristram signature of the underlying link $L^{\prime}$ is equal to

$$
\sigma_{L^{\prime}}(\omega)=\operatorname{sgn} \Re(\omega-1)+1=0 .
$$

This is the expected result since $L^{\prime}$ is slice in the ordinary sense.

\section{ACKNOWLEDGMENTS}

A part of this paper was done while the first author visited the Institut de Recherche Mathématique Avancée (Strasbourg) whose hospitality he thankfully acknowledges. He also wishes to thank Vladimir Turaev and Mathieu Baillif for valuable discussions. The second author thanks the Université Libre de Bruxelles and the Department of Geometry and Topology of the University of Zaragoza for their hospitality. He thanks in particular E. B. Artal and J. I. Cogolludo for exciting conversations.

\section{REFERENCES}

1. M. Atiyah, I. Singer, The index of elliptic operators. III, Ann. of Math. 87 (1968), 546-604. MR 0236952 (38:5245)

2. A. Casson, C. M. Gordon, Cobordism of classical knots in $S^{3}$, Printed notes. Orsay (1975).

3. A. Casson, C. M. Gordon, On slice knots in dimension three, Proc. Symp. in Pure Math. XXX (1978), Part 2, 39-53. MR0520521 (81g:57003)

4. D. Cimasoni, A geometric construction of the Conway potential function, Comment. Math. Helv. 79 (2004), no. 1, 124-146. MR2031702 (2005d:57005)

5. J. Conway, An enumeration of knots and links, and some of their algebraic properties, In Computational Problems in Abstract Algebra (Oxford, 1967), Proc. Conf. (Pergamon Press, Oxford, 1967), pp. 329-358. MR0258014(41:2661) 
6. D. Cooper, Signatures of surfaces with applications to knot and link cobordism, Ph. D. thesis, University of Warwick, 1982.

7. D. Cooper, The universal abelian cover of a link, Low-dimensional topology (Bangor, 1979), pp. 51-66, London Math. Soc. Lecture Note Ser., 48, Cambridge Univ. Press, Cambridge-New York, 1982. MR0662427 (83g:57002)

8. R. Crowell, D. Strauss, On the elementary ideals of link modules, Trans. Amer. Math. Soc. 142 (1969), 93-109. MR0247625 (40:889)

9. V. Florens, Signatures of colored links with application to real algebraic curves, J. Knot Theory Ramifications 14 (2005), 883-918. MR2187604

10. V. Florens, P. Gilmer, On the slice genus of links, Algebr. Geom. Topol. (2003), 905-920. MR2012958 (2004h:57006)

11. R. Fox, Some problems in knot theory, 1962 Topology of 3-manifolds and related topics (Proc. The Univ. of Georgia Institute, 1961) 168-176 Prentice-Hall, Englewood Cliffs, N.J. MR0140100 (25:3523)

12. P. Gilmer, Configurations of Surfaces in 4-manifolds, Trans. Amer. Math. Soc. 264 (1981), 353-380. MR0603768 (83h:57027)

13. P. Gilmer, On the Slice Genus of Knots, Invent. Math. 66 (1982), 191-197. MR0656619 (83g:57003)

14. P. Gilmer, C. Livingston, Discriminant of Casson-Gordon invariants, Math. Proc. Cambridge Philos. Soc. 112 (1992), no. 1, 127-139. MR1162937 (94e:57007)

15. C. M. Gordon, On the G-Signature theorem in dimension 4, Proceedings Okahoma Topology Conference 1978. MR0900251

16. C. M. Gordon, R. Litherland, K. Murasugi, Signatures of covering links, Canad. J. Math. 33 (1981), no. 2, 381-394. MR0617628(83a:57006)

17. R. Hartley, The Conway potential function for links, Comment. Math. Helv. 58 (1983), no. 3, 365-378. MR0727708 (85h:57006)

18. L. Kauffman, L. Taylor, Signature of links, Trans. Amer. Math. Soc. 216 (1976), 351-365. MR $0388373(52: 9210)$

19. J. Levine, Knot cobordism groups in codimension two, Comment. Math. Helv. 44 (1969), 229-244. MR0246314(39:7618)

20. J. Levine, Signature invariants of homology bordism with applications to links, Knots 90 (Osaka, 1990), 395-406, de Gruyter, Berlin, 1992. MR.1177436 (94g:57026)

21. J. Levine, Link invariants via the eta invariant, Comment. Math. Helv. 69 (1994), no. 1, 82-119. MR.1259607 (95a:57009)

22. A. Libgober, On the homology of finite abelian coverings, Topology Appl. 43 (1992), no. 2, 157-166. MR1152316 (93e:57003)

23. J. Murakami, On local relations to determine the multi-variable Alexander polynomial of colored links, Knots 90 (Osaka, 1990), 455-464, de Gruyter, Berlin, 1992. MR1177442 (93k:57020)

24. K. Murasugi, On a certain numerical invariant of link types, Trans. Amer. Math. Soc. 117 (1965), 387-422. MR0171275 (30:1506)

25. S. Orevkov, Link theory and oval arrangements of real algebraic curves, Topology 38 (1999), no. 4, 779-810. MR.1679799 (2000b:14066)

26. S. Orevkov, Plane real algebraic curves of odd degree with a deep nest, J. Knot Theory Ramifications 14 (2005), 497-522. MR2150745 (2006d:14068)

27. S. Orevkov, in preparation.

28. D. Rohlin, Two-dimensional submanifolds of four-dimensional manifolds, Funkcional. Anal. i Priložen. 5 (1971), no. 1, 48-60. MR0298684 (45:7733)

29. M. Sakuma, Homology of abelian coverings of links and spacial graphs, Can. J. Math. 47 (1995), no.1, 201-224. MR1319696 (96d:57008)

30. H. Seifert, Über das Geschlecht von Knoten, Mathematische Annalen 110 (1934), 571-592. MR 1512955

31. L. Smolinsky, A generalization of the Levine-Tristram link invariant, Trans. Amer. Math. Soc. 315 (1989), no. 1, 205-217. MR0931532 (89m:57021)

32. A. G. Tristram, Some cobordism invariants for links, Proc. Camb. Philos. Soc. 66 (1969), 251-264. MR0248854 (40:2104) 
33. H. Trotter, Homology of group systems with applications to knot theory, Ann. of Math. 76 (1962), no. 2, 464-498. MR0143201 (26:761)

34. O. Viro, Branched coverings of manifolds with boundary and link invariants I, Math. USSR Izvestia 7 (1973), 1239-1256. MR0370605(51:6832)

Department of Mathematics, University of California Berkeley, 970 Evans Hall, Berkeley, CALifornia 94720

E-mail address: cimasoni@math.berkeley.edu

Departamento Ãlgebra, Geometrã y Topologã , Universidad de Valladolid, Prado de la Magdalena S/n, 47011 Valladolid, Spain

E-mail address: vincent_florens@yahoo.fr

Current address: Section de Mathématiques, Université de Genève, 2-4 rue du Lièvre, Case Postale 64, 1211 Genève 4, Switzerland

E-mail address: vincent.florens@math.unige.ch 